\title{
Physics of runaway electrons in tokamaks
}

\author{
Boris N. Breizman* \\ Institute for Fusion Studies, The University of Texas, Austin, TX 78712, USA \\ Pavel Aleynikov ${ }^{\dagger}$ \\ Max-Planck-Institut für Plasmaphysik, Greifswald, Germany \\ Eric M. Hollmann团 \\ University of California - San Diego, \\ 9500 Gilman Dr, La Jolla, CA 92093-0417, USA \\ Michael Lehnen\$ \\ ITER Organization, Route de Vinon sur Verdon, \\ 13115 St Paul Lez Durance, France
}

\begin{abstract}
Of all electrons, runaway electrons have long been recognized in the fusion community as a distinctive population. They now attract special attention as a part of ITER mission considerations. This review covers basic physics ingredients of the runaway phenomenon and the ongoing efforts (experimental and theoretical) aimed at runaway electron taming in the next generation tokamaks. We emphasize the prevailing physics themes of the last 20 years: the hot-tail mechanism of runaway production, runaway electron interaction with impurity ions, the role of synchrotron radiation in runaway kinetics, runaway electron transport in presence of magnetic fluctuations, micro-instabilities driven by runaway electrons in magnetized plasmas, and vertical stability of the plasma with runaway electrons. The review also discusses implications of the runaway phenomenon for ITER and the current strategy of runaway electron mitigation.
\end{abstract}

\footnotetext{
* breizman@mail.utexas.edu

$\dagger$ pavel.aleynikov@ipp.mpg.de

$\ddagger$ ehollmann@ucsd.edu

$\S$ michael.lehnen@iter.org
} 


\section{CONTENTS}

\begin{tabular}{|lc}
\hline I. Introduction & 5 \\
\hline
\end{tabular}

$\begin{array}{lr}\text { II. Particle orbits } & 10\end{array}$

\begin{tabular}{ll}
\hline A. Guiding center orbits & 10
\end{tabular}

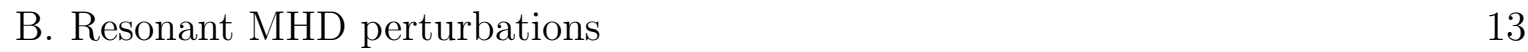

\begin{tabular}{ll}
\hline C. Orbit-averaged kinetic equation & 18
\end{tabular}

$\begin{array}{ll}\text { III. Elementary processes } & 22\end{array}$

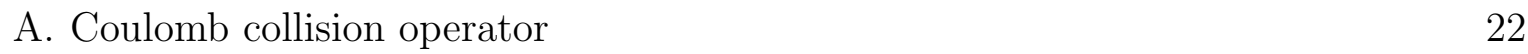

B. Electron-electron collisions and stopping power 23

$\begin{array}{ll}\text { C. Elastic scattering } & 27\end{array}$

D. Synchrotron radiation and radiation reaction force in the kinetic equation 31

E. Bremsstrahlung 33

\begin{tabular}{|ll}
\hline F. Pair production & 36
\end{tabular}

\begin{tabular}{|l|l|}
\hline IV. Thermal Quench & 37
\end{tabular}

A. Anomalous electron heat transport 38

\begin{tabular}{|ll}
\hline B. Enhanced losses of fast electrons & 39
\end{tabular}

C. Re-formation of flux-surfaces 40

\begin{tabular}{ll}
\hline D. Impurities & 41 \\
\hline
\end{tabular}

V. Primary runaway electrons

\begin{tabular}{ll}
\hline A. Dreicer source & 45
\end{tabular}

B. Compton effect and Tritium decay 48

C. Magnetic Reconnection 48

D. Hot-tail 50

VI. Runaway avalanche in a strong electric field $\quad 55$

\begin{tabular}{|ll}
\hline VII. Runaway kinetics at near-threshold electric field & 59 \\
\hline
\end{tabular}

\begin{tabular}{ll}
\hline VIII. Runaway current decay & 70 \\
\hline
\end{tabular}

\begin{tabular}{|l|l|}
\hline IX. Kinetic instabilities of runaway electrons & 77
\end{tabular} 
$\begin{array}{ll}\text { X. Experiment } & 84\end{array}$

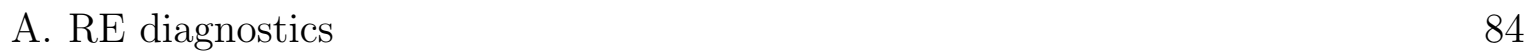

1. Bremsstrahlung 84

2. Synchrotron emission 86

\begin{tabular}{ll}
\hline 3. Cyclotron emission & 87
\end{tabular}

4. Line emission 88

5. Probe diagnostics 88

$\begin{array}{ll}\text { 6. IR thermography } & 89\end{array}$

7. Neutron emission 90

8. Laser scattering 90

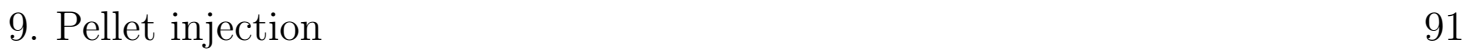

B. Startup and quiescent runaway electrons 91

C. Plateau runaway electrons and their dissipation 94

D. RE plateau equilibrium control and vertical stability 101

E. RE seed formation during TQ 101

F. RE prompt loss due to magnetic perturbations 103

G. Collisional suppression of CQ RE avalanche 105

H. RE energy deposition into the wall 106

$\begin{array}{ll}\text { XI. ITER Disruption Mitigation Strategy } & 109\end{array}$

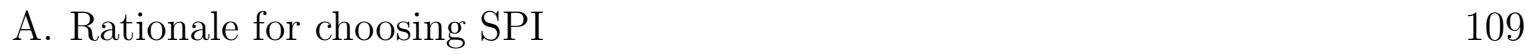

B. Disruption Mitigation Requirements for ITER 110

1. Provide sufficient radiation to reduce conductive energy losses during the thermal quench 110

2. Dissipate the magnetic energy through radiation 110

3. Provide a radiation distribution homogenous enough to prevent first wall melting 111

4. Prevent runaway formation during all phases of the disruption 112

5. Provide fast dissipation of the runaway electron energy 112

6. Injection quantities to respect upper limits from vacuum handling and

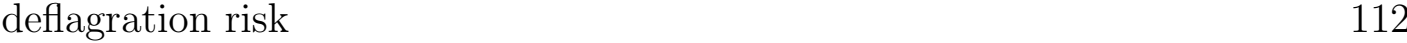

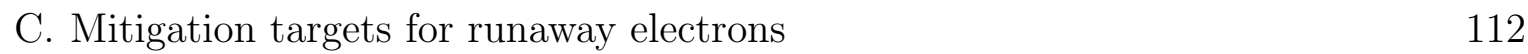


\begin{tabular}{ll}
\hline D. ITER DMS injection scenario & 113
\end{tabular}

\begin{tabular}{ll}
\hline E. ITER DMS layout & 114
\end{tabular}

\begin{tabular}{|ll}
\hline F. Runaway electron diagnostics in ITER & 115
\end{tabular}

\begin{tabular}{ll}
\hline G. Needs to validate the DMS design and strategy & 116
\end{tabular}

1. Avoidance of runaway electron formation 116

\begin{tabular}{ll}
\hline 2. Runaway electron energy dissipation & 117
\end{tabular}

\begin{tabular}{ll}
\hline H. Other runaway electron mitigation techniques & 117
\end{tabular}

\begin{tabular}{ll}
\hline XII. Summary & 120
\end{tabular}

$\begin{array}{ll}\text { Acknowledgments } & 123\end{array}$

$\begin{array}{ll}\text { References } & 123\end{array}$ 


\section{INTRODUCTION}

An inherent feature of a tokamak as a plasma confinement system is that the confined plasma has to carry a significant current, and the associated magnetic energy is greater than the kinetic energy of the current carrying particles. It is pertinent that the tokamak current is always much greater than the characteristic Alfvén current of $17 \mathrm{kA}$, by roughly three orders of magnitude in a fusion reactor. Bulk electrons are the current carriers in a fusion-grade plasma under nominal conditions, and the electron flow velocity is then much smaller than the electron thermal velocities. It is, however, possible that a small population of highly supra-thermal electrons becomes a significant or even the dominant current carrier. The underlying reason is that the friction force decreases with increasing speed for fast electrons, as shown in Fig. 1. The electrons can then undergo "unlimited" acceleration by an electric

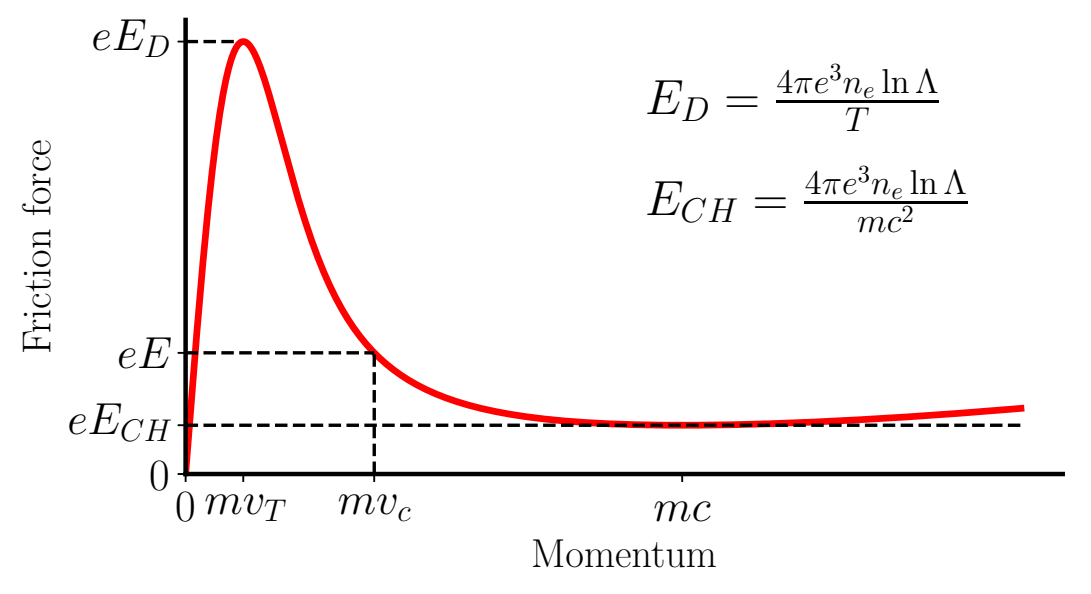

FIG. 1. Momentum-dependent friction force for a test electron in the plasma in comparison with the driving electric field $E$. To generate runaway electrons from a Maxwellian tail, the electric field has to exceed a critical Connor-Hastie field $E_{\mathrm{CH}}$. When $E>E_{\mathrm{CH}}$ electrons with momentum higher than the critical $m v_{c}$ enter runaway regime. All electrons enter the runaway regime when the electric field exceeds the Dreicer field $E_{D}$.

field, which is an effect predicted in 1925 in Ref. [1] and known now as runaway phenomenon. Runaway electrons have long been a spotlight topic in plasma research for both academic and practical reasons. They may trigger gas breakdown in strong electric fields, including those encountered in atmospheric lightning, they can be present in magnetic reconnection 
events, and they can be produced deliberately to create high-energy electron beams. Many aspects of electron beam physics and beam-plasma interactions pertain naturally to runaway electron behavior. One of such aspects is the excitation of waves by the fast electrons, either in a form of spontaneous non-thermal radiation or as a stimulated emission leading to electron-driven instabilities.

Collisional processes are much slower for the runaway electrons than they are for the bulk electrons. Their characteristically low collisionality does not allow runaway electrons to establish a Maxwellian distribution over the time-scales of interest. From the theory standpoint, runaway electrons require a kinetic treatment whereas the bulk is tractable with just the fluid moments.

Early theoretical studies have established two production mechanisms of the runaways: a diffusive leak of electrons from the Maxwellian tail into the runaway range [2] and knock-on collisions that multiply the runaway population exponentially [3]. The goal of those and subsequent studies was to describe the growth of a small runaway population in a given driving electric field without any feedback from the runaways on the field and without any collisional processes other than Coulomb collisions in a fully ionized plasma. The need to assess RE implications for tokamaks requires a broader approach that would allow to identify and integrate other relevant physics. In particular, high inductance of the system indicates that the electric field can hardly be viewed as a given. It changes dramatically in step with the runaway evolution whereas the current is more difficult to change. The problem thus requires a simultaneous description of the runaway kinetics and the evolving electric field as one of the research priorities. It is also necessary for an integrated physics analysis to cover very different time scales, such as collisional relaxation time in the bulk plasma, the timescale of runaway multiplication via avalanche mechanism, and the time scale of the plasma current decay. Runaway electrons are ubiquitous in the start-up plasmas during the electrical breakdown of the gas, but their current usually remains relatively small by the time plasma heats up and becomes a good conductor. They then dissipate gradually, because the low loop voltage is unable to support them in a $10 \mathrm{keV}$ temperature range. A sufficiently long time of the discharge shut-down in regular operational regimes can easily prevent reoccurrence of the runaways, but their reoccurrence becomes a clear danger when the shut-down time has to be very short in an emergency. Copious production of the runaway electrons can then be powered by the large stored energy of the poloidal magnetic field in 
the plasma [4, 5]. The potential damage of the plasma facing components of fusion reactors by these electrons is a matter of serious concern for all large tokamaks including ITER [6]. These mission-driven considerations are omnipresent in the runaway studies that we attempt to review in this paper now, 40 years after the then thorough review [7], 30 years after review focused on electron driven instabilities [8] and more than 20 years after the milestone work on avalanche production of the runaways [5].

The following physics themes have received particular attention in the last 20 years: the hot-tail mechanism of runaway production, the role of synchrotron radiation in runaway kinetics, runaway electron transport in presence of magnetic fluctuations, micro-instabilities driven by RE in magnetized plasmas, vertical stability of the plasma, with REs, and runaway electron interaction with impurity ions. All of them are essential physics elements of the disruption mitigation problem. These developments have addressed some of the outstanding research topics identified earlier in Ref. [9], such as the role of the hot tail and the ThomasFermi-based approach to fast electron scattering by the partially ionized high-Z impurities.

Rapid cooling of plasma thermal electrons is common at the beginning of the disruption events in tokamaks. This naturally links RE production to disruptions. An extensive empirical knowledge about disruptions and their control in existing machines is still insufficient to tell how to make reactor-size machines disruption-free. As a back-up "line of defense", a disruption mitigation system (DMS) is envisioned for ITER, and RE mitigation is a substantial part of this mission. Detailed overviews of ITER DMS strategies and the related research were published in 2015 in Ref. [10] and Ref. [11], respectively. The mitigation attempts rely on impurity injection into the plasma with the goal to radiate most of the stored energy without any structural damage. Massive gas injection and pellet injection are under consideration and experimental testing. There has been a significant progress in understanding the role of impurities in runaway kinetics, which includes improved description of RE interaction with bound electrons and partially screened nuclei in high-Z gases. This knowledge is essential for optimization of impurity content and impurity deposition technique because of the known dual role of impurities. They can either facilitate runaway production via bulk plasma cooling or help to dump the existing runaways via their enhanced scattering or via triggering MHD instabilities. It is, however, unclear at the moment whether the mitigation system can be sufficiently flexible to meet most of the physics and engineering constraints. The results of the ongoing experiments themselves are not immediately scalable 
to next-generation tokamaks, which raises concerns regarding DMS capabilities and runaway control, as summarised in Refs. [12, 13]. The problem requires a creative input from the first-principles theory and numerical simulations to develop a self-consistent picture of the bulk plasma and RE responses to impurity injection.

Experimental studies of runaway electrons have advanced significantly in recent years. Decades ago, runaway electrons were merely an occasional, and sometimes, a detrimental byproduct of the experiments. In contrast, nowadays, nearly every tokamak has a dedicated experimental time for the runaway research. Despite the fact that runaway electrons seem to be inevitable in ITER, it is hardly possible to learn enough about them from existing tokamaks that are significantly smaller in size, have lower current and are limited in pulse lengths. It is particularly difficult to have experimental settings in which the runaway behavior is robustly reproducible. Nonetheless, the latter problem has been solved successfully in many modern tokamaks. They now provide credible experimental data and new insights into runaway electron physics.

The experimental studies of runaway electrons are conducted in two distinct regimes: quiescent regimes and disruptive regimes. In quiescent runaway plasmas (QREs), a lowdensity plasma is run at lower and lower density until REs begin to appear; in this regime, the background hot plasma carries most of the current and only traces of relativistic electrons can be detected. In disruptive regimes, REs are created during a disruption and the current is quickly overtaken by the relativistic electrons. The QRE plasmas are beneficial from the diagnostic point of view, due to the long time scales, nearly steady-state conditions, and ability to use standard tokamak diagnostics (e.g. charge-exchange recombination) to diagnose the background plasma. On the other hand, the disruptive regimes, while more challenging to diagnose, allow study of many important processes, such as the vertical motion of the RE current channel and RE beam final loss when REs hit the vessel wall.

Although diagnosis of REs is challenging, especially in disruptive regimes, RE diagnostics have been expanded in recent years, as described in Section XA. Modern diagnostics, for instance, give a relatively good idea of the structure of the runaway electron distribution function, at least on a slow time scale, and permit some insights into the complex disruption processes, such as the complex poloidal structure of plasma line radiation during the thermal quench. Measurement of RE instabilities has improved, despite the challenging frequencies of $100 \mathrm{MHz}$ or more, enabling first detailed study of kinetic instabilities in REs. Synchrotron 
emission imaging has greatly improved, allowing the detailed structure of the most energetic REs to be measured.

Significant progress has been achieved in recent years in development of numerical tools. These tools include: continuous and Monte Carlo solvers for the kinetic equation, guiding center and full orbit codes, MHD codes, codes for modeling of kinetic instabilities, various synthetic diagnostic tools. Being focused on the physics of runaway electrons our review does not present technical descriptions of these numerical tools. We, however, give references to those tools when discussing corresponding physics. 


\section{PARTICLE ORBITS}

\section{A. Guiding center orbits}

The presence of strong magnetic field commonly justifies a guiding center description of electron motion. The guiding center theory implies that the spatial scale of the field is larger than the particle Larmor radius and the time-scales are longer than the electron gyro-period. The equilibrium field typically satisfies these conditions, but perturbed fields may violate them. It is convenient to use Lagrangian formalism for the guiding center motion. The guiding center Lagrangian was derived by Littlejohn [14] in a non-relativistic case and generalized to the relativistic case by Wimmel [15]. The relativistic guiding center Lagrangian has the form 1

$$
L=\frac{e}{c}\left[\mathbf{A}+\frac{m c}{e} u_{\|} \mathbf{b}\right] \dot{\mathbf{X}}+\frac{m^{2} c}{2 e} J_{\perp} \dot{\zeta}-e \Phi-m c^{2} \sqrt{1+\frac{u_{\|}^{2}}{c^{2}}+\frac{J_{\perp} B}{c^{2}}} .
$$

The dynamical variables in this Lagrangian are $\mathbf{X}, u_{\|}, J_{\perp}$, and $\zeta$. The vector potential $\mathbf{A}$, the scalar potential $\Phi$, the magnetic field absolute value $B$ and its unit vector $\mathbf{b}$ are all functions of $\mathbf{X}$ and time.

The variable $J_{\perp}$ is a relativistic adiabatic invariant related to magnetic moment $\mu$ :

$$
J_{\perp}=\mu \frac{2}{m} \sqrt{1+\frac{u_{\|}^{2}}{c^{2}}+\frac{J_{\perp} B}{c^{2}}} .
$$

The variable $u_{\|}$is related to the parallel velocity of the guiding center by

$$
(\mathbf{b} \cdot \dot{\mathbf{X}})=\frac{u_{\|}}{\sqrt{1+\frac{u_{\|}^{2}}{c^{2}}+\frac{J_{\perp} B}{c^{2}}}}
$$

as follows from one of the Lagrangian equations.

The dynamical variables $\mathbf{X}, u_{\|}, J_{\perp}$ and $\zeta$ are not canonical, but transformation to canonical variables is straightforward for orthogonal magnetic coordinates in which the vector potential of the equilibrium magnetic field and the magnetic field itself have the following covariant representation:

$$
\begin{aligned}
& \mathbf{A}=A_{\varphi} \nabla \varphi+A_{\theta} \nabla \theta, \\
& \mathbf{B}=B_{\varphi} \nabla \varphi+B_{\theta} \nabla \theta,
\end{aligned}
$$

${ }^{1}$ Note that $e$ is negative for electrons. 
where $\varphi$ and $\theta$ are the toroidal and poloidal angles, respectively, and $A_{\varphi}$ does not depend on angles at a flux surface. In a large aspect ratio tokamak with circular flux surfaces, the covariant components of the vector potential and the magnetic field are

$$
\begin{gathered}
A_{\varphi}=-B_{0} \int_{0}^{r} \frac{r d r}{q(r)} ; A_{\theta}=\frac{1}{2} B_{0} r^{2}-B_{0} \cos \theta \int_{0}^{r}\left(\frac{r^{2}}{R_{0}}+r \Delta^{\prime}+r \int_{0}^{r} \frac{\Delta^{\prime}}{r_{1}} d r_{1}\right) d r \\
B_{\varphi}=B_{0} R_{0} ; B_{\theta}=B_{0} \frac{r^{2}}{R_{0} q(r)}\left(1-\frac{r}{R_{0}} \cos \theta+\Delta^{\prime} \cos \theta-\int_{0}^{r} \frac{\Delta^{\prime}}{r_{1}} d r_{1} \cos \theta\right),
\end{gathered}
$$

where $B_{0}$ is the field on magnetic axis, $R_{0}$ is the major radius, $q(r)$ is the safety factor, and $\Delta(r)$ is the Shafranov shift.

Equations (4) transform the Lagrangian to

$$
L=P_{\varphi} \dot{\varphi}+P_{\theta} \dot{\theta}+P_{\zeta} \dot{\zeta}-H
$$

where

$$
\begin{aligned}
P_{\varphi} & \equiv \frac{e}{c}\left[A_{\varphi}+\frac{m c}{e} u_{\|} \frac{B_{\varphi}}{B}\right], \\
P_{\theta} & \equiv \frac{e}{c}\left[A_{\theta}+\frac{m c}{e} u_{\|} \frac{B_{\theta}}{B}\right], \\
P_{\zeta} & \equiv \frac{m^{2} c}{2 e} J_{\perp}
\end{aligned}
$$

and the Hamiltonian $H \equiv e \Phi+m c^{2} \sqrt{1+\frac{u_{\|}^{2}}{c^{2}}+\frac{J_{\perp} B}{c^{2}}}$ needs to be expressed in terms of $P_{\varphi}, P_{\theta}, P_{\zeta}$ and $\theta$.

The angles $\varphi, \theta, \zeta$ are now canonical coordinates and $P_{\varphi}, P_{\theta}, P_{\zeta}$ are the canonical momenta. The corresponding equations of motion are the Hamilton equations.

The orthogonal magnetic coordinates can always be introduced locally, but this coordinate system is inconvenient globally for many equilibrium configurations of interest, such as elliptically shaped equilibria where it involves singularity at magnetic axis. Also, the magnetic field lines are not necessarily straight in the orthogonal coordinates. For these reasons, numerical implementation of the orthogonal coordinates is challenging for realistic tokamaks. It is however possible to redefine the toroidal and poloidal angles and thus construct global straight field lines magnetic coordinates that preserve Hamiltonian structure of the guiding center equations [16]. 


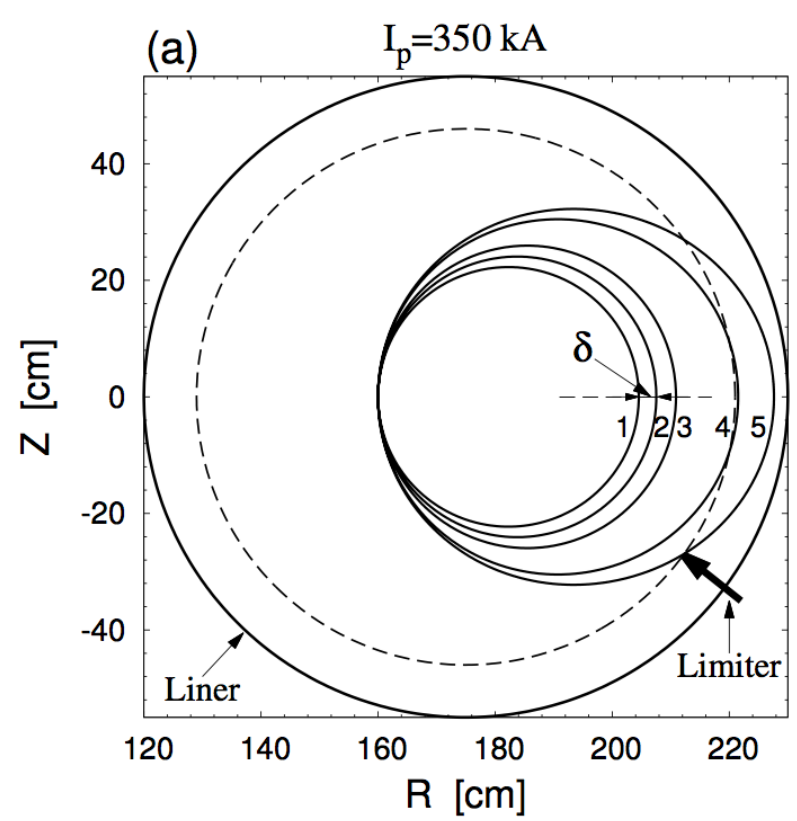

FIG. 2. Guiding-center orbits of electrons in TEXTOR corresponding to the different energies in the plasma with the plasma current $350 \mathrm{kA}$ and the toroidal field $2.5 \mathrm{~T}$ : curves 1-5 correspond to energies $\mathrm{E}=10 \mathrm{keV}, 10 \mathrm{MeV}, 20 \mathrm{MeV}, 40 \mathrm{MeV}$, and $46 \mathrm{MeV}$, respectively. Figure adopted from [17.

The guiding center motion in an equilibrium field of a tokamak is fully integrable. It preserves the toroidal magnetic moment $P_{\varphi}$, adabatic invariant $J_{\perp}$, and energy $W$. These conservation laws determine the shape of the particle orbit as

$$
W=e \Phi+\sqrt{m^{2} c^{4}+c^{2}\left(P_{\varphi}-\frac{e}{c} A_{\varphi}\right)^{2}\left(\frac{B}{B_{\varphi}}\right)^{2}+m^{2} c^{2} J_{\perp} B}=\text { const. }
$$

The unperturbed orbits are close to magnetic surfaces, which means that the shape of the orbit is to lowest order

$$
A_{\varphi}(\psi)=\frac{c}{e} P_{\varphi}=\text { const }
$$

where $\psi$ is the flux surface label. The runaway electrons have small pitch angles and move almost, albeit not exactly, along the magnetic field lines. Their orbits in the poloidal plane are generally shifted to the low-field side from the magnetic axis, as shown in Fig. 2, because electrons move opposite to the plasma current. 


\section{B. Resonant MHD perturbations}

The unperturbed motion is double periodic and characterised by the poloidal and toroidal transit frequencies $\omega_{\varphi}\left(P_{\varphi} ; W ; J_{\perp}\right)$ and $\omega_{\theta}\left(P_{\varphi} ; W ; J_{\perp}\right)$. Knowledge of these frequencies enables identification of resonant orbits (the ones that are most sensitive to perturbations). The resonance condition for a perturbation of frequency $\omega$ and toroidal number $n$ is

$$
\omega-n \omega_{\varphi}\left(P_{\varphi} ; W ; J_{\perp}\right)-l \omega_{\theta}\left(P_{\varphi} ; W ; J_{\perp}\right)=0
$$

where $l$ is an integer. For small perturbations, electron excursion from a resonant orbit scales as a square root of the perturbation amplitude, in contrast with the linear scaling for a non-resonant orbit [18]. A particle does not move far away from its unperturbed orbit as long as the resonant surfaces with different $n$ and $l$ values remain well separated in the constants of motion space. However, the motion changes sharply as soon as the neighbouring resonances overlap and the particle is then allowed to diffuse globally over a set of overlapping resonances.

The frequencies of MHD perturbations are typically much smaller than $\omega_{\varphi}\left(P_{\varphi} ; W ; J_{\perp}\right)$ and $\omega_{\theta}\left(P_{\varphi} ; W ; J_{\perp}\right)$ for relativistic electrons. Consequently, the resonance condition simplifies to

$$
-n \omega_{\varphi}\left(P_{\varphi} ; W ; J_{\perp}\right)-l \omega_{\theta}\left(P_{\varphi} ; W ; J_{\perp}\right)=0
$$

This simplification means that perturbations are effectively static and, thus, conserve the

energy of the particles. They change only the toroidal angular momentum $P_{\varphi}$, which leads to the transport of particles across the equilibrium magnetic surfaces. The resonances marked by Eq. (12) are closely related to rational magnetic surfaces, but they are not exactly there, because of the drift-orbit excursions. Consequently, stochastic diffusion of magnetic field lines does not necessarily cause the same diffusion of the fast electrons. In presence of static perturbations, fast electrons diffuse in $P_{\varphi}$ with $J_{\perp}$ and energy being conserved. Note that

$$
P_{\varphi} \approx-\frac{e B_{0}}{c} \int_{0}^{r} \frac{r d r}{q(r)}+m u_{\|}\left(R_{0}+r \cos \theta\right)
$$

in a large aspect ratio tokamak with circular flux surfaces. In this case, the equilibrium orbits of electrons with negligible pitch-angles $\left(J_{\perp}=0\right)$ deviate from the flux surface by roughly $m c u_{\|} q /\left(e B_{0}\right)$. The drift-orbit-averaged diffusion should, therefore, differ from the Rechester-Rosenbluth estimate [19] that neglects any orbit deviation from the field line. 
Calculation of the drift-orbit-averaged diffusion coefficient for passing particles in stochastic magnetic field can be found in Ref. [20]. This work also reconciles the differences between the results of earlier calculations. It is important to point out that the diffusion coefficient presented in Ref. [20] is formally a sum of delta-functions located at discrete resonances, which precludes global transport of particles unless some broadening of the delta-functions mixes the resonances into a continuous array. The sum can then be replaced by an integral over resonance labels.

Nonlinearity of the perturbed motion is commonly the dominant reason for resonance broadening [18]. The broadened resonances give rise to global quasi-linear diffusion when non-axisymmetric perturbations are large enough to overlap resonances with different $n$ and $l$ values. It is, however, not unusual that overlap of resonances with low to moderate values of $n$ and $l$ requires impractically large perturbations. Such resonances tend to form isolated phase space islands separated by Kolmogorov-Arnold-Moser (KAM) surfaces that act as transport barriers. The transport mechanism can then be a combination of particle convection within the islands and diffusion across the KAM surfaces. This suggests an advection-diffusion model for cross-field transport [21]. Although such model can reasonably replicate results of direct numerical simulations, it can hardly serve as an independent predictive tool. The problem is the inherent lack of universality in the transport coefficients.

To meet the resonance overlap condition, the perturbations should typically have large toroidal mode numbers. Such perturbations are not characteristic to linear MHD instabilities but they can be produced nonlinearly by the initial tearing mode. Simulations of existing experiments with nonlinear MHD codes NIMROD [22, 23] and JOREK [24] show that initially unstable long wavelength modes may eventually randomise magnetic surfaces within a large part of the plasma volume or even globally (see Figs. 3 and 4 ).

The magnetic field then loses its perfectly nested structure due to presumably random radial walk of every field line. The resulting diffusion coefficient for fast electrons should increase roughly as $(\delta B)^{2}$ with the perturbation level, but large uncertainty remains with regard to characteristic spatial scales and correlation lengths of the perturbations, which makes it difficult to quantify their impact on runaway confinement without speculative conjectures. It is still unfeasible for the existing codes and computers to simulate the randomized field directly, because of severe resolution requirements and the need for a kinetic rather than MHD description of the emerging short scales. This situation motivates numerous sensitivity 

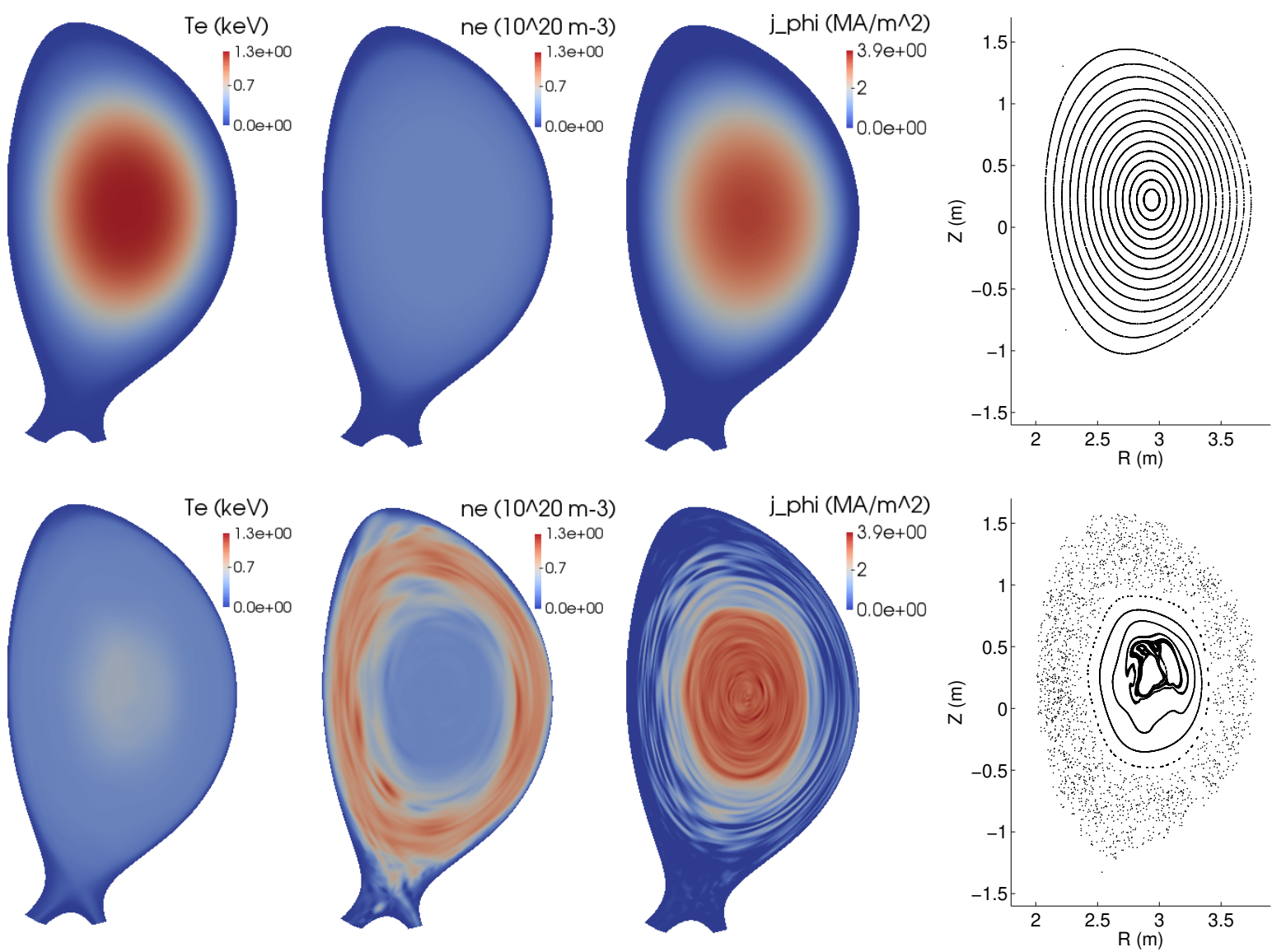

FIG. 3. Modeling of massive gas injection in JET. Poloidal cross sections of $T_{e}, n_{e}, j$, and Poincaré plots of the magnetic surfaces at the toroidal position of the injection at $t=0$ (upper row) and $t=6.2 \mathrm{~ms}$ (lower row). Figure adopted from Ref. [24].

studies of the fast electron transport to magnetic fluctuations produced by the MHD codes, created by external magnetic coils or to the arbitrarily postulated ones [21, 25, 31].

The efforts to quantify cross-field transport of runaway electrons under experimentally relevant conditions involves Monte Carlo simulations with either guiding center codes such as ANTS [27], ASCOT [32] or, more recently, a full orbit code KORC [33]. It is tempting to describe the outcome of such simulations in terms of a radial diffusion coefficient or particle confinement time, but this simplistic reduction can easily miss the critical sensitivity of the transport to the structure of nonlinear resonances and thus jeopardize the credibility of the results. The role of resonances needs, therefore, to be addressed explicitly in interpretation of the simulation results.

One of the issues resolved by numerical studies conclusively is whether external magnetic 


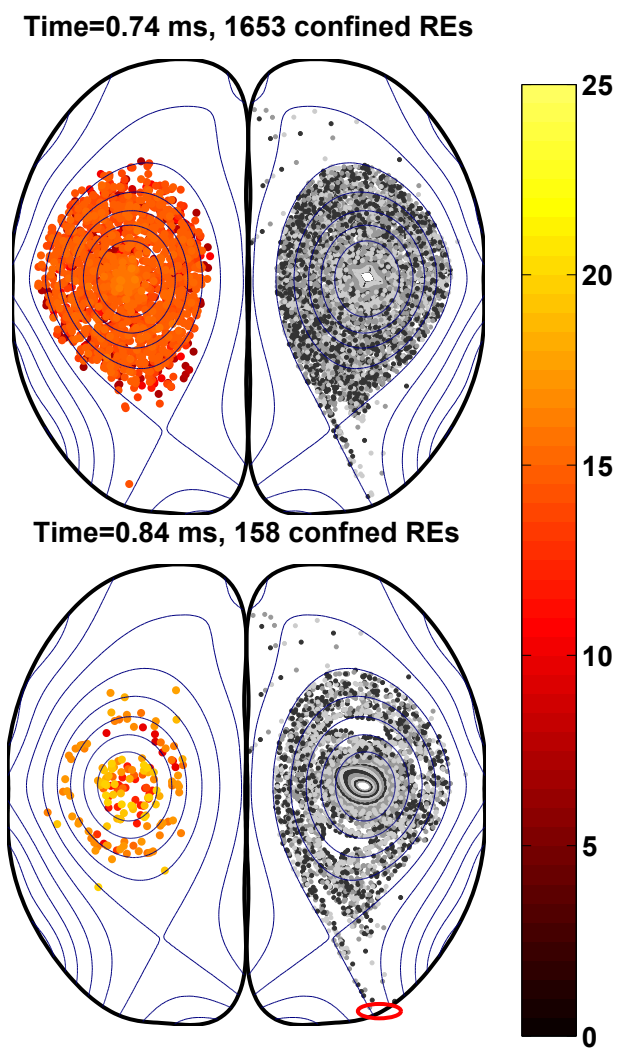

FIG. 4. Test particle simulation of runaway electron loss during thermal quench in DIII-D (shot 137620). Snapshots of the electron population before and after the thermal quench (0.74 ms and $0.84 \mathrm{~ms}$, respectively). Colored dots mark poloidal locations of the confined runaway electrons. At $0.84 \mathrm{~ms}$, the "outer ring" of runaway electrons lies mostly inside the flux tube defining the $2 / 1$ island. Each color represents electron energy in MeV. Grayscale dots on the right side are magnetic field line puncture plots with toroidally averaged poloidal flux contours superimposed. Left side plots with the color dots are mirror image cross-sections with identical poloidal flux contours. The circle in lower right indicates the poloidal location at which the escaping electrons strike the wall. Figure adopted from Ref. [28].

coils, also known as Resonant Magnetic Perturbation (RMP) coils, can be used to facilitate runaway losses [27, 34]. This may be an option for a small machine, like TEXTOR [35], but does not look practical for an ITER-size device. The reason is that the required short-scale perturbations decay fast with the distance from the coils and can extend to the plasma core only in a small machine. In larger machines, the resonances would overlap just in the outer part of the plasma, as seen in Fig. 5 that shows a Poincaré plot of the magnetic field 


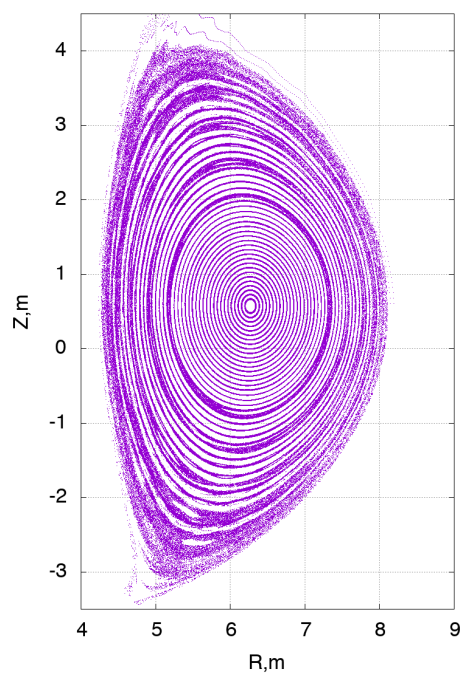

FIG. 5. Poincaré plot of ITER magnetic field with the outer magnetic surfaces destroyed by the resonant magnetic perturbation coils with the coil current of $90 \mathrm{kA}$. Figure adopted from Ref. [34].

structure in ITER when RMP is applied.

Particle tracing codes reveal that the contribution of the very short wavelength perturbations to transport is relatively small as a result of averaging along the guiding center orbit or full gyro-orbit. This is especially relevant to higher energy particles (and hence to runaways) that have larger orbit excursions from the equilibrium flux surfaces. Their diffusion coefficient should therefore decrease with energy. Depending on input parameters, one finds either global stochasticity or coexistence of stochastic areas and good magnetic surfaces within the plasma, which is instructive for understanding experimental data, but still not sufficient for conclusive first principle predictions of radial losses of the runaway electrons even when the codes simulate saturated regimes of plasma instabilities rather than just assess sensitivity to pre-specified perturbations. The co-existence of destroyed and undestroyed magnetic surfaces suggests that global diffusive losses may be hindered by transport barriers for runaway electrons, and the electrons would only escape in short pulses through statistically formed "turnstiles" 36]. 


\section{Orbit-averaged kinetic equation}

Particle motion in a tokamak is a combination of hamiltonian motion in the equilibrium magnetic field and a distortion of the equilibrium orbit by the perturbed fields and collisions. For a small population of runaway electrons, the resulting kinetic equation is linear in its distribution function and can be written schematically as

$$
\frac{\partial f}{\partial t}+\{H, f\}=\int C^{(6)}\left(Z, Z^{\prime}\right) f\left(Z^{\prime}\right) d^{(6)} Z^{\prime}
$$

with $H=H_{0}+H_{1}$, where $H_{0}$ is the Hamiltonian of the unperturbed motion, $H_{1}$ is the perturbed part of the Hamiltonian, $\{H, f\}$ is the Poisson bracket, $Z$ denotes a phase space point, the kernel $C^{(6)}\left(Z, Z^{\prime}\right)$ characterizes collisions, and the integral is over phase space volume. The perturbed Hamiltonian includes a regular inductive electric field as well as perturbations from randomly fluctuating fields. The latter are known to cause quasi-linear diffusion of the particles.

Equation (14) describes a particle distribution in 6-dimensional phase space, but the strong equilibrium magnetic field and toroidal symmetry of this field make it possible to reduce the dimensionality of the problem from 6 to 3 via a guiding center approximation and orbit averaging. The guiding center motion in the equilibrium magnetic field of a tokamak is fully integrable. It conserves particle energy, toroidal angular momentum and magnetic moment and can be characterized by canonical action-angle variables. The corresponding Hamiltonian $H\left(P_{\zeta} ; P_{\theta} ; P_{\varphi}\right)$ depends only on three canonical moments (actions) in this case. The actions are conserved quantities, and the conjugate canonical angles $\zeta, \theta, \varphi$ (related to but different from geometrical angles) are cyclic coordinates that characterize gyromotion, poloidal motion and toroidal motion respectively. An additional discrete index $\sigma$ (omitted here for brevity) labels passing and counterpassing particles.

The unperturbed guiding center motion is periodic in angles and has three characteristic frequencies, $\omega_{\zeta, \theta, \varphi}=\partial H / \partial P_{\zeta, \theta, \varphi}$. Equation (14) can thus be written as

$$
\frac{\partial f}{\partial t}+\omega_{\zeta} \frac{\partial f}{\partial \zeta}+\omega_{\theta} \frac{\partial f}{\partial \theta}+\omega_{\varphi} \frac{\partial f}{\partial \varphi}=\int C^{(6)}\left(Z, Z^{\prime}\right) f d P_{\zeta}^{\prime} d \zeta^{\prime} d P^{\prime}{ }_{\theta} d \theta^{\prime} d P^{\prime}{ }_{\varphi} d \varphi^{\prime}-\left\{H_{1}, f\right\}
$$

When the perturbed fields are small and the characteristic collision frequency is much lower

than $\omega_{\zeta, \theta, \varphi}$, the distribution function is a sum of a large slowly evolving part $\langle f\rangle$ that does not depend on angles and a small rapidly oscillating part $f_{\sim}$. The slowly evolving part will 
then satisfy a 3-dimensional equation with an angular averaged collision operator and two angular-averaged contributions from the perturbed fields (regular and random):

$$
\begin{aligned}
\frac{\partial\langle f\rangle}{\partial t} & =\int C^{(3)}\left(P_{\zeta, \theta, \varphi}, P_{\zeta, \theta, \varphi}^{\prime}\right)\langle f\rangle d P_{\zeta}^{\prime} d P^{\prime}{ }_{\theta} d P^{\prime}{ }_{\varphi}-\left\langle\left\{H_{1},\langle f\rangle\right\}\right\rangle-\left\langle\left\{H_{1}, f_{\sim}\right\}\right\rangle, \\
C^{(3)} & \equiv \frac{1}{(2 \pi)^{3}} \int C^{(6)}\left(P_{\zeta, \theta, \varphi}, \zeta, \theta, \varphi, P_{\zeta, \theta, \varphi}^{\prime}, \zeta^{\prime}, \theta^{\prime}, \varphi^{\prime}\right) d \zeta d \theta d \varphi d \zeta^{\prime} d \theta^{\prime} d \varphi^{\prime}
\end{aligned}
$$

Further simplification is possible in the case of small particle excursions from magnetic flux surfaces (zero orbit width approximation). This decouples different flux surfaces in Eq. (16) and reduces Eq. (16) to a two-dimensional equation on each flux surface. The zero orbit approximation means that radial transport of the particles can be neglected to lowest order. The orbit averaging in this case reduces to averaging over particle poloidal bounces on a given flux surface labeled by $P_{\varphi}$. Note that $P_{\varphi} \approx e A_{\varphi} / c$ in the zero orbit width approximation, and $C^{(3)}$ is a strongly peaked, delta-like function of $P_{\varphi}-P_{\varphi}^{\prime}$. The distribution function on the flux surface is a function of particle energy $\mathcal{E}$ and relativistic adiabatic invariant $J_{\perp} \equiv 2 e P_{\zeta} /\left(m^{2} c\right)$. Equation (16) then reduces to

$$
\begin{aligned}
& \frac{\partial\langle f\rangle}{\partial t}=\int C^{(2)}\left(P_{\varphi}, \mathcal{E}, J_{\perp}, \mathcal{E}^{\prime}, J_{\perp}^{\prime}\right)\langle f\rangle\left(P_{\varphi}, \mathcal{E}^{\prime}, J_{\perp}^{\prime}\right) d J_{\perp}^{\prime} d \mathcal{E}^{\prime}-\left\langle\left\{H_{1},\langle f\rangle\right\}\right\rangle-\left\langle\left\{H_{1}, f_{\sim}\right\}\right\rangle, \\
& C^{(2)}\left(P_{\varphi}, \mathcal{E}, J_{\perp}, \mathcal{E}^{\prime}, J_{\perp}^{\prime}\right)=\frac{m^{2} c}{2 e} \int \frac{C^{(3)}\left(P_{\varphi}, \mathcal{E}, J_{\perp}, P^{\prime}{ }_{\varphi}, \mathcal{E}^{\prime}, J_{\perp}^{\prime}\right)}{\omega_{\theta}\left(P^{\prime}{ }_{\varphi}, \mathcal{E}^{\prime}, J_{\perp}^{\prime}\right)} d P^{\prime}{ }_{\varphi}
\end{aligned}
$$

The collision operator in this equation splits into a sum of partial contributions from various elementary processes, which determines the following structure of Eq. (17),

$$
\frac{\partial\langle f\rangle}{\partial t}=\mathbb{C}_{1}\langle f\rangle+\mathbb{C}_{2}\langle f\rangle+\mathbb{C}_{3}\langle f\rangle+\mathbb{C}_{4}\langle f\rangle+\mathbb{E}\langle f\rangle+\mathbb{D}\langle f\rangle+\mathbb{S}\langle f\rangle
$$

where $\mathbb{C}_{1}\langle f\rangle$ is the electron drag term, $\mathbb{C}_{2}\langle f\rangle$ is the elastic scattering term, $\mathbb{C}_{3}\langle f\rangle$ describes the synchrotron radiation reaction, $\mathbb{C}_{4}\langle f\rangle$ describes bremsstrahlung losses, $\mathbb{E}\langle f\rangle$ is a contribution from the driving electric field, $\mathbb{D}\langle f\rangle$ describes quasi-linear diffusion in the presence of micro-instabilities, and $\mathbb{S}\langle f\rangle$ represents a particle source from knock-on collisions. We use the Euclid Math font in Eq. (18) to denote operators.

An explicit form of the bounce averaged kinetic equation describing Coulomb collisions, radiation friction, and a simplified knock-on collision source, can be found in Refs. [5, 37]. In these papers and elsewhere, a pitch angle parameter $\lambda$ is used as a variable instead of $J_{\perp}$. A finite orbit width correction would add a neoclassical radial transport term to the kinetic equation. We discuss specific contributions to the kinetic equation in more detail in subsequent sections of this review. 
Proper orbit averaging can be important for accurate quantitative predictions, especially for small-aspect-ratio machines, but most qualitative features of the runaway kinetics can already be revealed within a straight and uniform magnetic field set-up that we use in this review by default unless otherwise specified. Equations (19)-(25), listed below, show partial contributions to Eq. (18) for this set-up. These expressions imply that the background plasma is cold and fully ionized. The presence of partially ionized impurities does not change the structure of the listed operators but requires updated expressions for the stopping power and elastic collision frequency, as discussed further in Sections III B, III C.

- Electron drag

$$
\mathbb{C}_{1} f=\frac{1}{p^{2}} \frac{\partial}{\partial p} e E_{C H}\left(p^{2}+m^{2} c^{2}\right) f
$$

where $E_{C H} \equiv \frac{4 \pi e^{3} n_{e} \ln \Lambda}{m c^{2}}$ is the Connor-Hastie critical field.

- Elastic scattering

$$
\mathbb{C}_{2} f=\left(\frac{2 \pi e^{4}}{m^{2} c^{4}} c n_{\text {ion }} Z^{2} \ln \Lambda\right) \frac{m^{2} c^{2}}{p^{2}} \sqrt{1+\frac{m^{2} c^{2}}{p^{2}}} \frac{1}{\sin \theta} \frac{\partial}{\partial \theta} \sin \theta \frac{\partial f}{\partial \theta}
$$

- Synchrotron radiation reaction

$$
\mathbb{C}_{3} f=\frac{2 e^{4} B^{2}}{3 m^{3} c^{5}}\left[\frac{1}{p^{2}} \frac{\partial}{\partial p} \frac{p^{3} \sqrt{m^{2} c^{2}+p^{2}}}{m c} \sin ^{2} \theta f+\frac{m c}{p \sin \theta} \frac{\partial}{\partial \theta} \frac{p \cos \theta \sin ^{2} \theta}{\sqrt{m^{2} c^{2}+p^{2}}} f\right]
$$

- Bremsstrahlung

$$
\begin{aligned}
\mathbb{C}_{4} f & =n_{\text {ion }} c \int \frac{p_{1}}{\sqrt{p_{1}^{2}+m^{2} c^{2}}}\left(\frac{d \sigma_{b r}}{d^{3} \mathbf{p}}\right)_{\mathbf{p}_{1} \mathbf{p}} f\left(\mathbf{p}_{1}\right) d^{3} \mathbf{p}_{1} \\
& -n_{\text {ion }} c f(\mathbf{p}) \int \frac{p}{\sqrt{p^{2}+m^{2} c^{2}}}\left(\frac{d \sigma_{b r}}{d^{3} \mathbf{p}}\right)_{\mathbf{p p}_{1}} d^{3} \mathbf{p}_{1}
\end{aligned}
$$

where $\left(\frac{d \sigma_{b r}}{d^{3} \mathbf{p}}\right)_{\mathbf{p}_{1} \mathbf{p}}$ is the bremsstrahlung cross section (see Eq. 56p) integrated over directions of photon emission, the result of this integration can be found in Ref. [38]. This expression includes only electron-ion collisions, because the bremsstrahlung from electron-electron collisions is smaller for high-Z impurities.

- Electric field drive

$$
\mathbb{E} f=e E\left(-\frac{1}{p^{2}} \frac{\partial}{\partial p} \cos \theta p^{2} f+\frac{1}{\sin \theta} \frac{\partial}{\partial \theta} \frac{\sin ^{2} \theta}{p} f\right)
$$


- Quasilinear diffusion

$$
\mathbb{D} f=\left[\frac{1}{p^{2}} \frac{\partial}{\partial p} p^{2}\left(D_{p p} \frac{\partial f}{\partial p}+D_{p \theta} \frac{\partial f}{p \partial \theta}\right)+\frac{\partial}{p \sin \theta \partial \theta} \sin \theta\left(D_{\theta p} \frac{\partial f}{\partial p}+D_{\theta \theta} \frac{1}{p} \frac{\partial f}{\partial \theta}\right)\right]
$$

The diffusion coefficients are determined by the energy spectrum of the excited waves (see Section IX).

- Knock-on collisions

$$
\begin{aligned}
& \mathbb{S} f=\frac{n_{e}}{2 \pi^{2} m} \int \frac{p_{1}}{p} \frac{1}{\sqrt{p_{1}^{2}+m^{2} c^{2}}} \frac{1}{\sqrt{p^{2}+m^{2} c^{2}}}\left(\frac{d \sigma}{d \gamma}\right)_{\gamma \gamma_{1}} \times \\
& \frac{f\left(p_{1} ; \theta_{1}\right) d^{3} \mathbf{p}_{1}}{\sqrt{\sin ^{2} \theta \sin ^{2} \theta_{1}-\left(\cos \theta \cos \theta_{1}-\sqrt{\frac{\gamma-1}{\gamma+1}} \sqrt{\frac{\gamma_{1}+1}{\gamma_{1}-1}}\right)^{2}}}- \\
& \frac{n_{e}}{2 \pi^{2} m} f(p ; \theta) \int \frac{p}{p_{1}} \frac{1}{\sqrt{p_{1}^{2}+m^{2} c^{2}}} \frac{1}{\sqrt{p^{2}+m^{2} c^{2}}}\left(\frac{d \sigma}{d \gamma}\right)_{\gamma_{1} \gamma} \times \\
& d^{3} \mathbf{p}_{1} \\
& \sqrt{\sin ^{2} \theta \sin ^{2} \theta_{1}-\left(\cos \theta \cos \theta_{1}-\sqrt{\frac{\gamma_{1}-1}{\gamma_{1}+1}} \sqrt{\left.\frac{\gamma+1}{\gamma-1}\right)^{2}}\right.},
\end{aligned}
$$

where $n_{e}$ is the cold electron density; $\gamma \equiv \frac{1}{m c} \sqrt{p^{2}+m^{2} c^{2}}$ is the electron relativistic factor; $\frac{d \sigma}{d \gamma}$ is the Møller cross section (see Eq. (26)). 


\section{ELEMENTARY PROCESSES}

\section{A. Coulomb collision operator}

Binary collisions are generally described by the Boltzmann collision operator, which is quadratic in the particle distribution function. These collisions tend to establish a Maxwellian distribution. Small deviations from the Maxwellian can be studied with a linearized collision operator. Such linearization does not require the deviation to be small at every velocity. It is also justified when the distribution function includes a small tail of nonMaxwellian particles (say, runaway electrons), in which case linearization means that the tail particles collide just with the Maxwellian bulk rather than with each other. The linearized collision operator does not describe a closed system. Consequently, it does not conserve the number of tail particles, their momentum and energy. It must, however, return zero when applied to a Maxwellian distribution with the bulk plasma temperature. Additional physics factors allow further simplifications of the linearized collision operator.

First, the dominance of small-angle collisions enables the reduction of the integral operator to a Fokker-Planck-type operator with a large logarithmic factor in the collision frequency. The difference between the linear integral operator and the Fokker-Planck expression represents knock-on collisions that can give rise to the exponential growth of the fast particle population, albeit at a rate that is lower (by the large logarithm) than the small-angle collision rate. This growth rate can thus be calculated perturbatively, based on the lowest order long-lifetime solution of the Fokker-Planck equation.

Second, electron-ion collisions can reasonably be viewed as elastic scattering due to the small electron-ion mass ratio.

Third, the coefficients in the Fokker-Planck collision operator simplify significantly when particles of interest are much faster than the bulk electrons.

The last two simplifications were used in Ref. [39] for non-relativistic particles and in Ref. [40] in a relativistic case.

A linearized Fokker-Planck operator that applies to both, slow (thermal) and fast (runaway range) velocities is presented in Ref. [41]. This combined operator is suitable for simultaneous calculation of Spitzer resistivity and runaway dynamics in an electric field that is significantly lower than the Dreicer field. 


\section{B. Electron-electron collisions and stopping power}

A quantum-mechanical description of relativistic electron collisions based on the Dirac equation was developed by Møller in Refs. [42, 43]. The resulting differential cross-section for a fast electron collision with a target electron that is initially at rest has the form (see, for example, [44])

$$
\begin{aligned}
& \frac{d \sigma}{d \gamma}=\frac{2 \pi r_{e}^{2}}{\gamma_{0}^{2}-1}\left[\gamma_{0}^{2}\left(\frac{1}{\gamma-1}\right)^{2}+\gamma_{0}^{2}\left(\frac{1}{\gamma_{0}-\gamma}\right)^{2}\right. \\
& \left.+1-\frac{2 \gamma_{0}-1}{\gamma_{0}-1}\left(\frac{1}{\gamma-1}+\frac{1}{\gamma_{0}-\gamma}\right)\right]
\end{aligned}
$$

where $r_{e}$ is the classical electron radius and $\gamma_{0}$ is the incident electron relativistic factor. The first two terms in Eq. (26) represent the classical Rutherford cross-section; the remaining terms are due to the exchange interaction.

In hot plasmas, impurities are typically fully ionized, so that all bulk electrons are free. In contrast, cold post-disruption plasmas contain both free electrons and bound electrons residing in partially ionized impurities. The presence of bound electrons is essential when runaways are mitigated by massive injection of material. Collisions with free and bound electrons have apparent differences and need to be considered separately.

Collisions with bound electrons - Given the dominance of small energy exchanges in Coulomb collisions, the resulting energy losses can be viewed as continuous and described in terms of a stopping power derived by Bethe in Ref. [45]. The stopping power in the form derived in the paragraph "Ionization losses of fast particles" in [44],

$$
\frac{d \mathcal{E}}{d t}=-\frac{2 \pi e^{4} n_{\text {bound }}}{m c \beta}\left[\ln \frac{m^{2} c^{4}\left(\gamma^{2}-1\right)(\gamma-1)}{2 I^{2}}-\left(\frac{2}{\gamma}-\frac{1}{\gamma^{2}}\right) \ln 2+\frac{1}{\gamma^{2}}+\frac{(\gamma-1)^{2}}{8 \gamma^{2}}\right]
$$

where $I$ is the mean excitation potential, $\beta$ is the incident electron velocity normalized to the speed of light, and $\gamma=1 / \sqrt{1-\beta^{2}}$. Equation (27) can be obtained from 26 by imposing a proper constraint on the minimum energy transfer to bound electrons [46]. Note that this equation does not include the small density-effect correction associated with the dielectricresponse function of the medium. More information about the density effect can be found in Refs. [47].

The mean excitation potential for neutral gases is approximately $I \approx(10 \mathrm{eV}) Z_{0}$ [48], where $Z_{0}$ is the charge of the nucleus, which ensures accuracy of Eq. (27) to within about 
10\%. In numerical calculations, it might be preferable to use either Eq. (27) and experimentally verified ionization potentials with summation over impurity species of interest or stopping power data provided in Ref. [47]. A convenient approximation of [47] data for the ionization potentials of Argon, Neon and their ions can be found in Ref. [49].

Equation (27) is similar but still somewhat different from the earlier Bethe formula [50] for ionization losses of a heavy fast particle

$$
\frac{d \mathcal{E}}{d t}=-\frac{2 \pi e^{4} n_{\text {bound }}}{m c \beta}\left[\ln \frac{4\left(m c^{2}\right)^{2} \gamma\left(\gamma^{2}-1\right)(\gamma-1)}{I^{2}}-2 \beta^{2}\right] \text {. }
$$

Apart from the different numerical factor in the large argument of the logarithm, there is also a different $\gamma$-dependence for the electrons. This is because the incident electron has a stronger deflection in an individual collision than a heavy particle. The stopping power is therefore somewhat lower for an electron than for a heavy particle with the same incident velocity.

Collisions with free electrons - Collisions with free electrons give a stopping power with a logarithmic factor that involves plasma frequency $\omega_{p}=\sqrt{4 \pi n_{f r e e} e^{2} / m}$ and represents shielding of the incident particle field in cold plasmas [46, 51]

$$
\frac{d \mathcal{E}}{d t}=-\frac{2 \pi e^{4} n_{\text {free }}}{m c \beta}\left[\ln \frac{m^{2} c^{4}\left(\gamma^{2}-1\right)(\gamma-1)}{2 \hbar^{2} \omega_{p}^{2} \gamma^{2}}-\left(\frac{2}{\gamma}-\frac{1}{\gamma^{2}}\right) \ln 2+1+\frac{(\gamma-1)^{2}}{8 \gamma^{2}}\right] \text {. }
$$

The presence of the excitation potential in Eq. (27) and the Planck constant in Eq. (29) indicate that both expressions require a quantum mechanical derivation. The difference between the logarithmic factors in Eqs. (27) and 29) can be explained qualitatively at semiclassical level by noting that the logarithmic factor is essentially twice the classical Coulomb $\operatorname{logarithm}, \ln \Lambda$, defined as $\ln \Lambda \equiv \ln \left(\rho_{\max } / \rho_{\min }\right)$, where $\rho_{\min }$ and $\rho_{\max }$ are the minimum and the maximum values of the impact parameter. The value of $\rho_{\min }$ is the same for the free and the bound electrons, because it corresponds to the closest possible collision. The maximum impact parameter for the bound electrons is roughly $\frac{\beta c \hbar}{\sqrt{1-\beta^{2}} I}$, which means that the duration of an ionizing collision should be shorter than the characteristic orbital period of the bound electron $\hbar / I$. For collisions with free electrons, the skin depth $\beta c / \omega_{p}$ determines the maximum impact parameter. This is why $\hbar \omega_{p} \gamma$ replaces $I$ under the logarithm. Note that the large logarithmic factor in Eq. (29) differs from the classical Coulomb logarithm for non-relativistic plasma in which $\rho_{\max }$ is the Debye length. 
For $10 \mathrm{MeV}$ electrons and $10^{20} \mathrm{~m}^{-3}$ plasma density, we have $\ln \Lambda_{\text {free }} \approx 21$, whereas $\ln \Lambda_{\text {bound }}$ in Eq. (27) for $10 \mathrm{MeV}$ electrons in Argon is approximately 12. The large logarithmic factors are commonly viewed as constants, because of their relatively weak dependence on arguments. This suggests the following frequently used practical estimate for the total stopping power [5]:

$$
\frac{d \mathcal{E}}{d t}=-\frac{4 \pi e^{4}}{m c \beta} \ln \Lambda_{\text {free }}\left(n_{\text {free }}+\frac{1}{2} n_{\text {bound }}\right),
$$

although it is apparently not as accurate as expressions 27) and (29).

Close collisions (knock-ons) - Unlike collisions with small energy exchanges, the rarer events with larger exchanges cannot be described adequately in terms of the stopping power and be represented by a differential operator in the kinetic equation. The borderline between the small and large exchanges is somewhat fuzzy, which is usually acceptable, because of the weak (logarithmic) dependence of the stopping power on the choice of this borderline. In general, the large logarithm requires some adjustment to avoid double counting, as detailed in Ref. 52 .

The knock-on collisions between the primary fast electrons and the immobile target electrons create a one-step access for the secondary electrons to the runaway regime. Such collisions are characterized by the Møller cross section (26).

The outgoing electron moves at an angle $\theta_{\gamma}$ with respect to the incident electron velocity. The energy and momentum conservation laws ascertain that

$$
\cos \theta_{\gamma}=\sqrt{\frac{\gamma-1}{\gamma+1}} \sqrt{\frac{\gamma_{0}+1}{\gamma_{0}-1}}
$$

Equations (26) and (31) determine the source $\mathbb{S}$ (production rate) of the outgoing electrons in phase space in terms of the distribution function $\left(f_{0}\right)$ of the incident electrons:

$$
\mathbb{S}=n_{f u l l} c \int \frac{p_{0}}{2 \pi \sqrt{p_{0}^{2}+1}} \frac{p}{p^{2} \sqrt{p^{2}+1}} \frac{d \sigma}{d \gamma} \delta\left[\left(\mathbf{n} \cdot \mathbf{n}_{0}\right)-\cos \theta_{\gamma}\right] f_{0}\left(p_{0} ; \mathbf{n}_{0}\right) p_{0}^{2} d p_{0} d \Omega_{0}
$$

where $\mathbf{n}_{0}$ and $\mathbf{n}$ are the unit vectors along the momenta of the incident and the outgoing electrons, respectively, $d \Omega_{0}$ is the solid angle element for $\mathbf{n}_{0}$, and $n_{\text {full }}$ is the total density of free and bound electrons (assuming that the energy exchange in close collisions exceeds the ionization energy for the bound electrons). Each scattering event can be viewed as a production of two outgoing electrons (described by the source $\mathbb{S}$ ) and the disappearance of the incident electron, which requires a corresponding sink term in the kinetic equation. Implementation of this bookkeeping is straightforward in Monte-Carlo simulations [53] but 
requires some extra care within a continuous formulation to preserve particle conservation properties in the kinetic equation. Reference [52] provides such a continuous formulation along with a procedure for solving the kinetic equation numerically.

The bookkeeping problem is particularly simple when the primary electrons are ultrarelativistic and the secondary electrons of interest have much lower energies. One can then formally use Eqs. (26) and (31) in the limit of $\gamma_{0} \rightarrow \infty$ to describe production of just the secondary electrons, which automatically eliminates the need to consider a sink term separately. This simplification is justified when the electric field exceeds the runaway avalanche threshold significantly. The resulting source term has been introduced in Ref. [5] with an additional assumption that the primary electrons have a negligibly small pitch angle. Reference [54] offered an amended source term that did not use the $\gamma_{0} \rightarrow \infty$ limit and could, therefore, describe collisions with comparable energies of the primary and secondary electrons. This, however, left an open question of adding a proper sink. Also, that amended source still implied that all primary electrons had negligibly small pitch angles. The latter was then a necessary compromise to make computations affordable. Modern computational resources enable exact modeling of the source for a gyro-averaged distribution function.

The argument of the $\delta$-function in Eq. (32) involves the azimuthal angle of the electron gyro-motion. To perform gyro-averaging of the $\delta$-function, we rewrite it as

$$
\delta\left[\left(\mathbf{n} \cdot \mathbf{n}_{0}\right)-\cos \theta_{\gamma}\right]=\delta\left[\cos \theta \cos \theta_{0}+\sin \theta \sin \theta_{0} \cos \varphi-\cos \theta_{\gamma}\right]
$$

and average this expression over the azimuthal angle $\varphi$ to obtain

$$
\left\langle\delta\left[\left(\mathbf{n} \cdot \mathbf{n}_{0}\right)-\cos \theta_{\gamma}\right]\right\rangle_{\varphi}=\frac{1}{\pi} \frac{1}{\sqrt{\sin ^{2} \theta \sin ^{2} \theta_{0}-\left(\cos \theta \cos \theta_{0}-\cos \theta_{\gamma}\right)^{2}}},
$$

or, using notations from the bounce-averaged kinetic equation [5, 37],

$$
\left\langle\delta\left[\left(\mathbf{n} \cdot \mathbf{n}_{0}\right)-\cos \theta_{\gamma}\right]\right\rangle_{\varphi}=\frac{1}{\pi} \frac{1}{\sqrt{b^{2} \lambda \lambda_{0}-\left(\sigma \sqrt{1-b \lambda} \sigma_{0} \sqrt{1-b \lambda_{0}}-\cos \theta_{\gamma}\right)^{2}}},
$$

where $b \equiv \frac{B}{B_{\max }}$ with $B$ and $B_{\max }$ being the local magnetic field and the maximum magnetic field on the primary electron trajectory, respectively; $\lambda \equiv \frac{\sin ^{2} \theta}{b}$, and $\sigma \equiv \pm 1$ denotes "co" and "counter" motion with respect to the electric field.

Unlike the simplified source from Ref. [5], the exact source (32) has a finite angular spread. Figure 6 shows the distribution functions 32 of secondary electrons produced by 

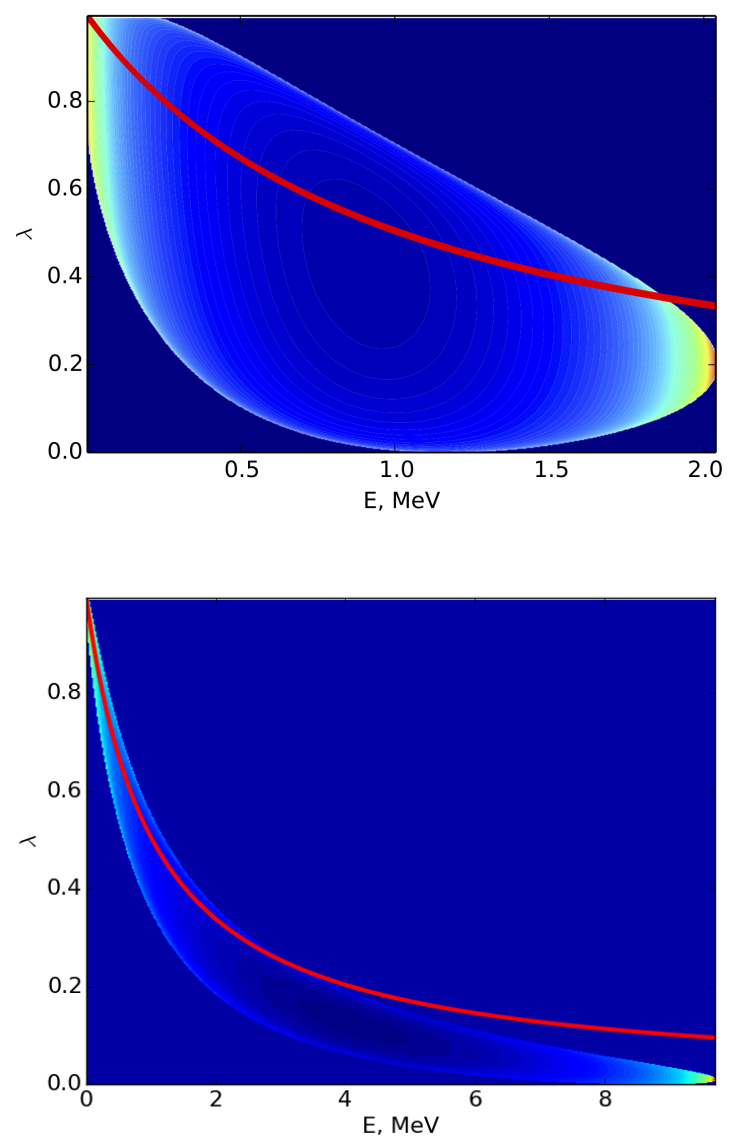

FIG. 6. Comparison of the exact knock-on collision source term with a simplified source from Ref. [5. Color-coded plots present the exact source given by Eqs. (32), (35) on magnetic axis $(b=1)$ for primary electrons with $\gamma_{0} \approx 5, \lambda_{0}=0.2$ (upper panel) and $\gamma_{0} \approx 20, \lambda_{0}=0$ (lower panel). The red curves represent the simplified source.

primary electrons with $\gamma_{0} \approx 5$ and $\gamma_{0} \approx 20$. The red curve in the figure marks the location of the simplified source from Ref. [5]. We note that the angular distribution obtained with Eq. (32) differs considerably from the simplified model in the lower energy case $\left(\gamma_{0} \approx 5\right)$, but the difference is less significant at higher energy $\left(\gamma_{0} \approx 20\right)$.

\section{Elastic scattering}

The Coulomb collision cross section scales as $Z^{2}$ with the charge of the scattering centre. As a result, elastic scattering of electrons is mostly due to their collisions with ions in plasma with high- $Z$ ions. This aspect is of particular relevance to runaway electron kinetics, because 
high-energy electrons can probe not just the total ion charge, but also the internal structure of the partially ionized impurities where shielding of the positive central charge by the bound electrons is less pronounced. The differential cross section of elastic scattering is given by

$$
\begin{aligned}
& d \sigma=\frac{1}{4}\left(\frac{e^{2}}{m c^{2}}\right)^{2} \frac{m^{4} c^{4}}{p^{4}} \frac{1}{\sin ^{4}(\theta / 2)}\left(1+\frac{p^{2}}{m^{2} c^{2}} \cos ^{2}(\theta / 2)\right)\left|Z_{0}-F(q)\right|^{2} d \Omega, \\
& q \equiv \frac{2 p}{\alpha m c} \sin (\theta / 2)
\end{aligned}
$$

where $F(q)$ is the bound electron form-factor, $p$ is the incident electron momentum, $\theta$ is the scattering angle, and $\alpha \equiv \frac{e^{2}}{\hbar c}$ is the fine structure constant. Equation (36) modifies the Mott cross section [44, 55] by including the bound electron form-factor. A quantum mechanical derivation of this equation involves the Born approximation, which is appropriate in the most important case of small-angle collisions. The corresponding collision operator for elastic scattering has a differential structure as a result:

$$
\mathbb{C}_{e l}=\frac{\nu_{e l}}{2} \frac{m^{2} c^{2} \sqrt{p^{2}+m^{2} c^{2}}}{p^{3}} \frac{1}{\sin \theta} \frac{\partial}{\partial \theta} \sin \theta\left(\frac{\partial}{\partial \theta} F\right),
$$

where

$$
\nu_{e l}=n_{i o n} c \frac{p^{4}}{m^{2} c^{2}\left(p^{2}+m^{2} c^{2}\right)} \int(1-\cos \theta) d \sigma
$$

is a characteristic elastic collision frequency for the relativistic electrons. The multiplier $\frac{p^{4}}{m^{2} c^{2}\left(p^{2}+m^{2} c^{2}\right)}$ in Eq. 38 is chosen in such a way that $\nu_{e l}$ has only weak (logarithmic) dependence on particle momentum.

The form-factor $F(q)$, which is given by

$$
F(q)=\int \rho_{\text {bound }}(r) \frac{\sin (q r)}{q r} 4 \pi r^{2} d r
$$

and the resulting collision frequency for different ion species have been evaluated in Refs. [49, 56] based on the Thomas-Fermi theory for bound electron density $\rho_{\text {bound }}(r)$. Ref. [49] presents a systematic numerical evaluation of $F(q)$, whereas Ref. [56] involves a conjectural interpolation for $F(q)$ between the limiting cases of small and large momenta. Ref. [49] provides the following expression for $\nu_{e l}$ :

$$
\begin{aligned}
\nu_{e l} & =n_{i o n} c \frac{4 \pi e^{4}}{m^{2} c^{4}} X, \\
X & \equiv Z^{2} \ln \Lambda+2 Z_{0} Z I_{1}(y)+Z_{0}^{2} I_{2}(y), \\
y & \equiv \frac{2 p}{m c \alpha Z_{0}^{1 / 3}},
\end{aligned}
$$


where $\ln \Lambda$ is the classical Coulomb logarithm and coefficients $I_{1}(y)$ and $I_{2}(y)$ grow logarithmically with $y$ as

$$
\begin{aligned}
& I_{1}(y) \approx I_{1}\left(y_{*}\right)+\left(\xi / Z_{0}\right) \ln \left(y / y_{*}\right) \\
& I_{2}(y) \approx I_{2}\left(y_{*}\right)+\left(\xi / Z_{0}\right)^{2} \ln \left(y / y_{*}\right) .
\end{aligned}
$$

Table I presents these coefficients for $y=26$ for ions with different number of bound electrons $(\xi)$. These values can be used in Eq. 41) (as $I_{1}\left(y_{*}\right)$ and $I_{2}\left(y_{*}\right)$ with $y_{*}=26$ ) to evaluate the coefficients for arbitrary momentum for various ions of elements lighter than argon. Note that single value of $\xi / Z_{0}$ corresponds to different ions of different elements, for instance, data from $\xi / Z_{0}=9 / 18$ corresponds to ions $\mathrm{He}^{+1}, \mathrm{Be}^{+2}, \mathrm{C}^{+3}, \mathrm{Ne}^{+5}$ and $\mathrm{Ar}^{+9}$.

TABLE I. Values of $I_{1}\left(y=y_{*}=26\right)$ and $I_{2}\left(y=y_{*}=26\right)$ integrals for ions with different number

\begin{tabular}{|c|c|c|c|c|c|}
\hline$\xi / Z_{0}$ & $I_{1}(26)$ & $I_{2}(26)$ & $\xi / Z_{0}$ & $I_{1}(26)$ & $I_{2}(26)$ \\
\hline $18 / 18$ & - & 2.5632 & $9 / 18$ & 1.1898 & 0.4632 \\
\hline $17 / 18$ & 2.9781 & 2.2181 & $8 / 18$ & 1.0096 & 0.3467 \\
\hline $9 / 10$ & 2.7663 & 1.9706 & $4 / 10$ & 0.8722 & 0.2676 \\
\hline $16 / 18$ & 2.7091 & 1.9061 & $7 / 18$ & 0.8382 & 0.2494 \\
\hline $15 / 18$ & 2.4587 & 1.6240 & $6 / 18$ & 0.6757 & 0.1701 \\
\hline $8 / 10$ & 2.3187 & 1.4711 & $3 / 10$ & 0.5830 & 0.1308 \\
\hline $14 / 18$ & 2.2220 & 1.3697 & $5 / 18$ & 0.5229 & 0.1078 \\
\hline $3 / 4$ & 2.1094 & 1.2526 & $1 / 4$ & 0.4504 & 0.0826 \\
\hline $13 / 18$ & 1.9969 & 1.1414 & $4 / 18$ & 0.3808 & 0.0612 \\
\hline $7 / 10$ & 1.9117 & 1.0588 & $2 / 10$ & 0.3273 & 0.0468 \\
\hline $12 / 18$ & 1.7816 & 0.9379 & $3 / 18$ & 0.2511 & 0.0291 \\
\hline $11 / 18$ & 1.5756 & 0.7577 & $2 / 18$ & 0.1368 & 0.0098 \\
\hline $6 / 10$ & 1.5369 & 0.7254 & $1 / 10$ & 0.1160 & 0.0073 \\
\hline $10 / 18$ & 1.3785 & 0.5998 & $1 / 18$ & 0.0447 & 0.0013 \\
\hline
\end{tabular}
of bound electrons $(\xi)$

More recently, a Density Functional Theory (DFT) and a numerical tool EXCITING [57] have been used in Ref. [58] to evaluate $F(q)$ and $\nu$. This reassessment introduces small corrections to the interpolation formula of Ref. [56] without revealing any significant difference from the calculations based on the Thomas-Fermi model. Figure 7 shows comparison of the factor $X$ of Eqs. (40) evaluated using Eq. (6) from [58] and using Eqs. (41). The difference in the arguments of the large logarithms is within 10\%, which gives even smaller correction to the collision frequency.

At a qualitative level, elastic collisions of fast electrons with partially ionized impurities can be described classically by a scattering potential with two different shielding radii

$$
\Phi=|e| \frac{Z_{0}-Z}{r} \exp \left(-r / r_{1}\right)+|e| \frac{Z}{r} \exp \left(-r / r_{2}\right)
$$




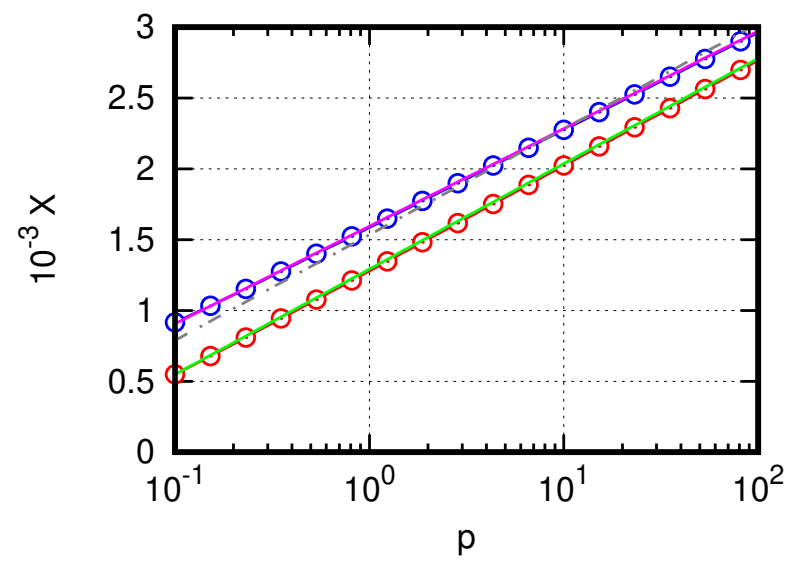

FIG. 7. Coefficient $X$ as determined from Eqs. 40 41) (solid) and Ref. [58] (markers) for $\mathrm{Ar}^{+}$(red, green) and $\mathrm{Ar}^{5+}$ (blue, purple). Grey dashed-dotted line is from the qualitative estimate Eq. (45) for Argon atom.

In this expression, $r_{1}$ and $r_{2}$ are the shielding radii for the bound electrons and free electrons, respectively. The corresponding scattering angle for a particular collision is then

$$
\Delta \theta=\left.\frac{e}{m c p} \int_{-\infty}^{\infty} d t \frac{\partial \Phi}{\partial r}\right|_{r=\sqrt{\rho^{2}+c^{2} t^{2} p^{2} /\left(1+p^{2}\right)}}
$$

where $\rho$ is the impact parameter and $u$ the electron velocity.

The collision frequency $\nu$ is simply the rate of pitch-angle spreading of the initially cold electron beam due to collisions with various impact parameters per unit time:

$$
\nu_{e l}=\pi n_{i o n} c \frac{p^{4}}{m^{2} c^{2}\left(p^{2}+m^{2} c^{2}\right)} \int(\Delta \theta)^{2} \rho d \rho .
$$

The logarithmically diverging integral in this expression needs to be truncated at the lower limit by choosing $\rho_{\min } \sim \hbar /(m c p)$. We retain only the "large logarithm" terms in Eq. (44) and take into account that $r_{1}$ is much smaller than $r_{2}$. These simplifications reduce Eq. (44) to

$$
\nu_{e l} \approx n_{\text {ion }} c \frac{4 \pi e^{4}}{m^{2} c^{4}}\left[Z^{2} \ln \frac{r_{2}}{r_{1}}+Z_{0}^{2} \ln \frac{r_{1}}{2 \rho_{\min }}+2\left(Z_{0}-Z\right) \ln 2\right] .
$$

The shielding radii $r_{1}$ and $r_{2}$ in this expression are the Thomas-Fermi radius, $r_{1}=$ $\hbar^{2} /\left(m e^{2} Z_{0}^{1 / 3}\right)$, and the Debye radius. Expression 445 is consistent with Ref. [49], but it differs parametrically from Eq. (26) of Ref. [59] in which $\rho_{\min }$ in the large logarithm $\ln \left(r_{1} / 2 \rho_{\text {min }}\right)$ appears to be the distance where the potential energy is comparable to the 
kinetic energy, whereas the quantum limitation $\left(\rho_{\min } \sim \hbar /(m c p)\right)$ is actually more restrictive and gives a somewhat lower collision frequency as a result. The logarithmic factor $\ln \left(r_{2} / r_{1}\right)$ in Eq. (45) is roughly the Coulomb logarithm and it is typically greater than $\ln \left(r_{1} / 2 \rho_{\min }\right)$, but the large $Z_{0}^{2}$ factor can easily be decisive for how to choose the correct "effective charge" for impurity ions.

\section{Synchrotron radiation and radiation reaction force in the kinetic equation}

In classical physics, acceleration of a charged particle is known to produce electromagnetic radiation, and the corresponding radiated power for a non-relativistic electron in its instantaneous rest-frame is [60]

$$
P=\frac{2}{3} \frac{e^{2}}{m^{2} c^{3}}\left(\frac{d \mathbf{p}}{d t}\right)^{2}
$$

Reference [61] provides an elegant generalization of Eq. (46) to the relativistic case. It points out that the radiated power is an invariant, because radiated energy and elapsed time transform in the same manner under Lorentz transformations. This implies that $(d \mathbf{p} / d t)^{2}$ needs to be replaced by the invariant combination

$$
(d \mathbf{p} / d s)^{2}-(d \mathcal{E} / d s)^{2} / c^{2}
$$

where $d s=d t \sqrt{1-v^{2} / c^{2}}$ is the differential of proper time. Of the two terms in expression (47) only $(d \mathbf{p} / d s)^{2}$ contributes to the radiated power when an electron moves in a static magnetic field. In a spatially uniform field, Eq. (46) simplifies to,

$$
P=\frac{2 e^{4} B^{2}}{3 m^{4} c^{7}} p^{2} c^{2} \sin ^{2} \theta
$$

where $\theta$ is the pitch angle. This radiation is entirely due to electron gyro-motion. When the field lines are curved, there is also a so-called curvature radiation resulting from the fieldaligned motion. The curvature radiation can be important in astrophysical conditions [62], but it is less significant in tokamaks [63. A more detailed description of synchro-curvature radiation, including its spectral distribution, angular distribution and polarization, can be found in Refs. 64 66].

For relativistic electrons, synchrotron radiation is mostly at high harmonics of the electron gyro-frequency. These frequencies are typically higher than the electron plasma frequency, 
which makes them immune from shielding by the plasma. Also, the runaway electrons themselves do not reabsorb this radiation, because of their low density. The system can thus be viewed as optically thin, and the outgoing radiation can provide a valuable diagnostic information about runaway electrons.

The emitted photons have much lower energies than the runaway electrons, which means that radiation reaction can be included in the kinetic equation for runaway electrons as a non-Hamiltonian friction force. The corresponding term in the kinetic equation must preserve the number of particles and can therefore be written as a divergence of flux in momentum space,

$$
\frac{\partial f}{\partial t}=\frac{1}{p^{2}} \frac{\partial}{\partial p} p^{2} A_{1} f+\frac{1}{p \sin \theta} \frac{\partial}{\partial \theta} \sin \theta A_{2} f
$$

where the distribution function is azimuthally symmetric due to gyro-averaging. To specify functions $A_{1}(p ; \theta)$ and $A_{2}(p ; \theta)$ in Eq. (49), we take into account that the energy loss rate per particle must satisfy Eq. (48) (we herein ignore corrections due to curvature radiation) and that the radiating particles preserve their parallel velocities. The latter becomes apparent if one notes that the radiating electron (irrespective of its energy) preserves its zero parallel velocity in a uniform magnetic field if the initial velocity is perpendicular to the field. This means that the parallel velocity of the radiating electron is preserved in any inertial reference frame that moves along the magnetic field. Conservation of the parallel velocity requires

$$
\int \frac{p \cos \theta}{\sqrt{m^{2} c^{2}+p^{2}}} \frac{\partial f}{\partial t} p^{2} \sin \theta d p d \theta=0
$$

which gives the following relation between $A_{1}(p ; \theta)$ and $A_{2}(p ; \theta)$ :

$$
A_{1} \cos \theta \frac{m^{2} c^{2}}{\left(m^{2} c^{2}+p^{2}\right)}=A_{2} \sin \theta .
$$

We next consider the rate of energy loss:

$$
\frac{\partial}{\partial t} \int \mathcal{E} f p^{2} \sin \theta d p d \theta=\int \mathcal{E}\left[\frac{1}{p^{2}} \frac{\partial}{\partial p} p^{2} A_{1} f+\frac{1}{p \sin \theta} \frac{\partial}{\partial \theta} \sin \theta A_{2} f\right] p^{2} \sin \theta d p d \theta .
$$

The second term on the right-hand side vanishes due to integration over $\theta$. The first term gives (upon integration by parts)

$$
\frac{\partial}{\partial t} \int \mathcal{E} f p^{2} \sin \theta d p d \theta=-\int \frac{\partial \mathcal{E}}{\partial p} A_{1} f p^{2} \sin \theta d p d \theta
$$

This expression must agree with Eq. (48), which determines $A_{1}$ :

$$
A_{1}=\frac{2 e^{4} B^{2}}{3 m^{2} c^{4}} \frac{p \sqrt{p^{2}+m^{2} c^{2}}}{m^{2} c^{2}} \sin ^{2} \theta .
$$


Taken together, Eqs. 49), (51) and (54) give [37]

$$
\frac{\partial f}{\partial t}=\frac{2 e^{4} B^{2}}{3 m^{3} c^{5}}\left[\frac{m c}{p^{2}} \frac{\partial}{\partial p} p^{2} \frac{p \sqrt{m^{2} c^{2}+p^{2}}}{m^{2} c^{2}} \sin ^{2} \theta f+\frac{m c}{p \sin \theta} \frac{\partial}{\partial \theta} \frac{p \cos \theta \sin ^{2} \theta}{\sqrt{m^{2} c^{2}+p^{2}}} f\right] .
$$

Equation (55) is more accurate than the earlier Eq. (49) of Ref. [67], because it automatically conserves the number of particles². This equation has later been re-derived in Refs. [69, 70]. Synchrotron losses can easily exceed the Coulomb collision losses if there is an efficient pitch-angle scattering mechanism for the runaways. Such scattering can be due to (1) high$\mathrm{Z}$ impurities and (2) micro-instabilities driven by the runaways.

\section{E. Bremsstrahlung}

The elastic scattering of relativistic electrons on ions is actually an approximation, in which radiation is neglected. This simplification is justified for collisions with large impact parameters, whereas closer collisions can be accompanied by non-negligible emission of photons. In non-magnetized plasmas this emission (bremsstrahlung) can be the dominant energy loss mechanism for ultra-relativistic electrons. A cross-section for the process in which an electron is scattered on a heavy particle with emission of a single photon was first derived in Refs. [71-73]. This cross section, for an electron with initial momentum $\mathbf{p}$ scattered into the solid angle $d o^{\prime}$ with momentum $\mathbf{p}^{\prime}$ and emitting a photon with momentum $\mathbf{k}$ into the solid angle $d o_{k}$, is given in Ref. [44] as

$$
\begin{aligned}
& d \sigma=\frac{Z^{2} \alpha r_{e}^{2}}{4 \pi} \frac{d \omega}{\omega} \frac{p^{\prime} m^{4}}{p q^{4}} d o_{k} d o^{\prime} \times \\
& \times\left\{\frac{q^{2}}{\kappa \kappa^{\prime} m^{2}}\left(2 \varepsilon^{2}+2 \varepsilon^{\prime 2}-q^{2}\right)+q^{2}\left(\frac{1}{\kappa^{\prime}}-\frac{1}{\kappa}\right)^{2}-4\left(\frac{\varepsilon}{\kappa^{\prime}}-\frac{\varepsilon^{\prime}}{\kappa}\right)^{2}+\right. \\
& \left.+\frac{2 \omega q^{2}}{m^{2}}\left(\frac{1}{\kappa^{\prime}}-\frac{1}{\kappa}\right)-\frac{2 \omega^{2}}{m^{2}}\left(\frac{\kappa}{\kappa^{\prime}}+\frac{\kappa^{\prime}}{\kappa}\right)\right\},
\end{aligned}
$$

where $\kappa=\varepsilon-(\mathbf{n} \cdot \mathbf{p}), \kappa^{\prime}=\varepsilon^{\prime}-\left(\mathbf{n} \cdot \mathbf{p}^{\prime}\right), \mathbf{n}=\mathbf{k} / \omega$ and $\mathbf{q}=\mathbf{p}^{\prime}+\mathbf{k}-\mathbf{p}$ is the momentum transferred to an immobile ion. Equation (56) is derived using the Born approximation, implying that $v / c \gg Z \alpha$, which in practice is well satisfied for relativistic particles and typical ions. In Eq. (56) we retained the notation of the book [44] to preserve its familiarity. In what follows we go back to our notation, with the symbol prime (') referring to the scattered particle.

\footnotetext{
${ }^{2}$ The approximate operator used in Ref. 67] was however sufficient to treat high-energy electrons considered therein. Neverthless, the error was corrected in Refs. [37, 68]
} 
In the ultra-relativistic limit $\left(\gamma, \gamma^{\prime} \gg 1\right)$ angular distribution of the photons has a specific shape of a narrow forward cone with an opening angle $\sim 1 / \gamma$. This feature can be used to infer the characteristics of the runaway electron distribution function when the angular distribution of the bremsstrahlung radiation is measured [74 76].

The spectral distribution of the radiation is obtained from Eq. (56) via integration over the secondary electron directions $\left(d o^{\prime}\right)$ and the photon directions $\left(d o_{k}\right)$. In the ultra-relativistic limit, this spectral distribution is

$$
d \sigma=4 \alpha Z^{2} r_{e}^{2} \frac{d \omega}{\omega}\left[\left(1+\frac{\gamma^{\prime 2}}{\gamma^{2}}\right) \phi_{1}+\frac{\gamma^{\prime}}{\gamma} \phi_{2}\right]
$$

where $\phi_{1}=\ln \left(2 \gamma \gamma^{\prime} m c^{2} /(\hbar \omega)\right)-1$ and $\phi_{2}=-\frac{2}{3} \phi_{1}$. Note that the resulting expression given by Eq. (57), as well as Eq. (56), diverges when $\omega \rightarrow 0$, suggesting that an infinite number of photons is emitted with zero energy - a phenomenon called "infrared catastrophe" [77. Consequently, multi-photon processes dominate in this limit rather than single-photon emission. The total "infrared" part of the cross-section then converges to the classical elastic scattering cross-section.

It follows from Eq. (57) that the energy loss of an electron is mostly due to emission of "hard" photons with $\hbar \omega \sim \gamma m c^{2}$. This means that bremsstrahlung has to be treated as a non-differential process when accounting its back-reaction on the particles [78]. Yet, a useful estimate of the average energy loss (so called radiative stopping power) can be obtained by integrating Eq. (57) over all photon energies. The resulting friction force $d \mathcal{E} / d x=$ $\int_{0}^{\gamma-1} n_{Z} w d \sigma$ is

$$
\frac{d \mathcal{E}}{d x}=-4 \alpha n_{Z} Z(Z+1) r_{e}^{2} m c^{2}(\gamma-1)(\ln (2 \gamma)-1 / 3) .
$$

We have herein replaced one of $Z$ with $(Z+1)$ to account for collisions with $Z$ electrons in plasma (the cross section for electron-electron collision coincides with Eq. (56) in the ultra-relativistic limit [44]).

Figure 8 shows the radiative stopping power defined by Eq. (58) for Argon $(Z=18)$ (grey solid curve) in comparison with the collisional (dotted curve) and radiative (dashed curve) stopping power data for Argon from Ref. [47]. As seen from the presented curves, Eq. (58) overestimates losses in high energies. The difference is due to partial screening of the ion charge by the bound electrons.

The effect of screening on bremsstrahlung is less significant than that on elastic collisions due to the much smaller impact parameters involved. Yet, the screening needs to be taken 


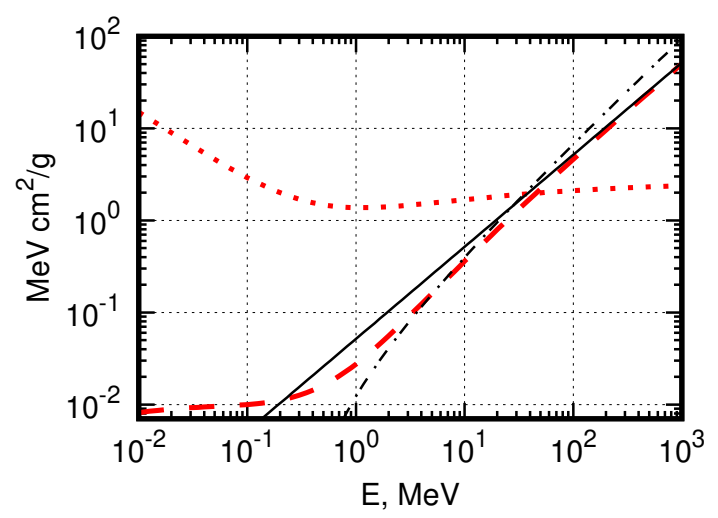

FIG. 8. Collisional (dotted curve) and radiative (dashed curve) losses of fast electron in Argon from the data of Ref. [47] and radiative losses calculated without (dashed-dotted) and with (solid) screening effects taken into account, i.e. using Eqs. (58) and (60), respectively.

into account when the impact parameter $(r)$ for a collision is comparable or greater than the atomic radius $\left(a_{Z} \approx Z^{-1 / 3} r_{B o h r} \equiv Z^{-1 / 3} r_{e} / \alpha^{2}\right)$. To account for screening, the functions $\phi_{1}$ and $\phi_{2}$ in Eq. (57) are to be replaced with:

$$
\phi_{1}=\ln \left(191 Z^{-1 / 3}\right), \phi_{2}=-\frac{2}{3} \phi_{1}+1 / 9 .
$$

The corresponding radiative stopping power

$$
\frac{d \mathcal{E}}{d x}=-4 \alpha n_{Z} Z(Z+1) r_{e}^{2} m c^{2}(\gamma-1)\left(\ln \left(191 Z^{-1 / 3}\right)-1 / 18\right)
$$

is shown in Fig. 8 for Argon with a dashed-dotted line that agrees better with the ESTAR data [47].

Indeed, the impact parameter for a $50 \mathrm{MeV}$ electron emitting half its energy in a collision with Argon is roughly $r \approx \frac{\hbar}{m c} \frac{\gamma\left(\gamma m c^{2}-\hbar \omega\right)}{\hbar \omega} \approx \frac{\gamma}{\alpha} r_{e} \approx a_{Z}$ (see, for example, Ref. [64]), indicating the need to account for screening.

The non-differential nature of bremsstrahlung losses suggests that bremsstrahlung can be viewed as a process that knocks an electron out of the runaway regime in a single collision and can be treated as a sink in calculation of the runaway avalanche growth rate. 


\section{F. Pair production}

Multi-MeV runaway electrons can create electron-positron pairs via collisions with nuclei. The cross-section of this process in the ultra-relativistic limit has the form [79]:

$$
\sigma=\frac{28}{27 \pi} r_{e}^{2} Z^{2} \alpha^{2} \ln ^{3} \gamma
$$

This asymptotic expression implies that the incident-particle energy is much greater than the $1 \mathrm{MeV}$ pair production threshold. Calculations of the cross section for lower energies, from the threshold to $100 \mathrm{MeV}$, are presented in Ref. [80]. They generally give lower values of the cross section than the formally applied Eq. (61). A more recent Ref. [81] states that the cross section is actually by a factor of 4 lower than that of Ref. [80], but Ref. [81] does not explain this disturbing discrepancy.

The ratio of the pair production to the total bremsstrahlung cross section is roughly $\approx$ $\frac{\alpha}{4 \pi} \ln ^{2} \gamma$, ensuring insignificance of the pair production as a loss mechanism for any accessible runaway electron energies. Pair production can still be of interest for diagnostic purposes.

As estimated in Ref. 82, the post-disruption runaways colliding with plasma ions will produce $10^{13}-10^{14}$ confined runaway positrons. Although this number is so small that it is difficult to detect these positrons experimentally, it is much larger than in any typical positron experiment. Ref. [81] discusses operational parameters required for positron detection via annihilation radiation during runaway production in tokamak discharges, and Ref. 83] provides an anticipated spectrum of positron synchrotron radiation that might be detectable. Synchrotron radiation from positrons has a distinct polarization, which can help to detect their signal. 


\section{THERMAL QUENCH}

Generation of runaway electrons during plasma disruptions is closely tied to the presumed disruption scenario. The phenomenology presented in current literature bases on several decades of experimental observations and, at a highest level, suggests the following sequence of events for both "intentional" and unintentional disruptions: Thermal Quench (TQ) a rapid loss of the plasma kinetic energy, Current Quench (CQ) - a subsequent decay of the plasma current, and, sometimes, Runaway Plateau - a regime when the majority of the current is carried by RE. This sequence of events is illustrated schematically in Fig. 9 .

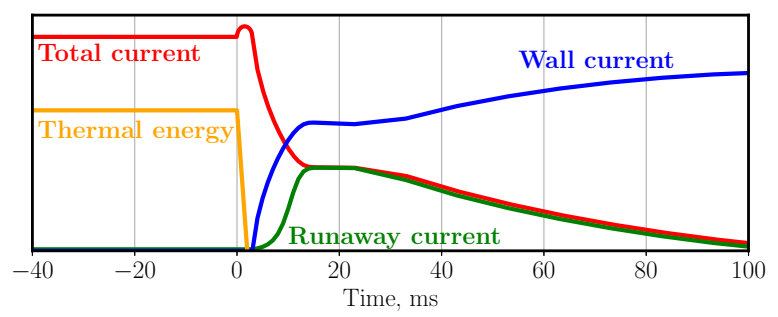

FIG. 9. Anticipated evolution of the plasma thermal energy (orange), total plasma current (red), runaway electron current (green) and the wall current (blue) in ITER. At 20ms the density of the background plasma increases to facilitate mitigation of the runaway electron current.

The reasons for fast cooling of an initially "healthy" plasma, as well as the exact routes of such cooling, vary between tokamaks as well as between experiments on a particular tokamak. A thorough statistical analysis of the events leading to a TQ in JET, for example, can be found in [84]. There are two major factors that are common to many experiments: impurity influx (or their intentional injection) resulting in strong radiative losses (line-radiation), and global MHD events that enhance heat transport via stochastization of the magnetic field lines. These two factors are often interdependent, i.e. the impurity influx leads to global MHD events and, vice-versa, an MHD event can bring impurities into the plasma from the Plasma Facing Components (PFC). The limiting cases of both impurity- and MHDdominated TQs have been observed experimentally. A fast killer-pellet injection in DIII-D $(\approx 400 \mathrm{~m} / \mathrm{s})$, reported in Ref. [85], shows very low MHD activity during the TQ, in contrast with the case of slow $(\approx 200 \mathrm{~m} / \mathrm{s})$ killer-pellet cases in similar conditions. On the other hand, some of the JET experiments reveal [86] that the plasma temperature quickly recovers after 
the MHD-dominated TQ, indicating a very low impurity content in the post-TQ plasma.

The thermal quench commonly leads to runaway electron production. This is because the toroidal current cannot change considerably on the fast TQ time-scale; the current changes on a longer (inductive) time-scale. Given that the plasma conductivity $(\sigma)$ drops down together with the temperature drop,

$$
\sigma=\frac{T^{3 / 2}}{\pi Z e^{2} m^{1 / 2} \ln \Lambda},
$$

the inductive electric field has to rise in order to preserve the current. The enhanced electric field makes it easier for the electrons to become runaways. In what follows, we discuss some aspects of theTQ in more detail.

\section{A. Anomalous electron heat transport}

The presence of MHD activity enables anomalous heat transport due to magnetic perturbations. In the case of global stochastization of the field lines, such heat transport is a powerful loss mechanism leading to a fast temperature drop. As discussed by Ward and Wesson in [87], in the case of ignorable radiative losses the resulting temperature $\left(T_{e}\right)$ is governed by the heat-diffusion equation

$$
\frac{1}{r} \frac{\partial}{\partial r} r n_{e} D \frac{\partial T_{e}}{\partial r}=\frac{j^{2}}{\sigma}
$$

where $n_{e}, j$ are the plasma density and current, respectively, and $D$ is a thermal diffusion coefficient, that depends on the level of magnetic perturbations $(\delta B / B)$ and their correlation length $(L)$. The ideal MHD perturbations tend to be elongated with a correlation length comparable to the connection length $q R$. The relatively long correlation length $(\sim q R)$ is typically longer than the mean free path $\left(\lambda_{e}\right)$ of the post-TQ plasma electrons

$$
\lambda_{e} \equiv v_{e} \tau_{e}
$$

where $v_{e}$ is the electron thermal velocity and $\tau_{e}$ denotes the electron collisional time [88]

$$
\tau_{e} \equiv \frac{3}{4 \sqrt{2 \pi}} \frac{m_{0}^{1 / 2} T_{e}^{3 / 2}}{e^{4} n_{e} \ln \Lambda} .
$$

In the limiting case of $L \gg \lambda_{e}$, the diffusivity in the stochastic magnetic field is simply a product of the parallel thermal conductivity $\chi_{\|}$[88] and the squared relative amplitude of 
magnetic perturbations $(d B / B)$, i.e.

$$
D=\chi_{\|}\left(\frac{\delta B}{B}\right)^{2} \approx 6.1 \frac{T_{e} \tau_{e}}{m}\left(\frac{\delta B}{B}\right)^{2} .
$$

An approximate solution of Eq. (63) gives the following dependence of the central temperature on the fluctuation level [87]:

$$
T_{e}[e V] \approx 15\left(B_{\theta a} Z_{e f f} \frac{B}{\delta B}\right)^{2 / 5},
$$

where $B_{\theta a}$ is the value of the poloidal magnetic field in Tesla at the plasma edge.

Equation (67) predicts the balance temperature for ITER in the range of $350 \mathrm{eV}$ to $60 \mathrm{eV}$ for the fluctuation amplitudes of $0.1 \%$ to $10 \%$. Although these temperatures do raise the inductive electric field easily above the critical Connor-Hastie level, they do not necessarily render runaway production. This is because (1) the same magnetic perturbations shorten the confinement time of the fast electrons, and (2) the plasma can quickly reheat after restoration of the broken magnetic surfaces.

\section{B. Enhanced losses of fast electrons}

It is instructive to estimate the energy gain of a collisionless test particle while this particle diffuses to the boundary through the broken magnetic surfaces. We assume that the perturbations create a set of overlapped resonances (see Section II B). Then the diffusion coefficient for such a particle is roughly

$$
D_{1}=L v_{e}\left(\frac{\delta B}{B}\right)^{2}
$$

where $L$ is the axial correlation length of the perturbations and $v_{e}$ is the particle velocity. The corresponding confinement time is $\tau \approx a^{2} / D_{1}$, where $a$ is the machine minor radius. The resulting ratio of the energy gain to plasma temperature (Eq. 67) ) is

$$
\frac{m v^{2}}{T_{e}} \approx \frac{e E v_{e} \tau}{T_{e}} \approx \frac{a}{Z_{e f f} L}\left(\frac{B}{\delta B}\right),
$$

where the inductive electric field is calculated using Spitzer conductivity for the temperature determined by Eq. (67). It follows from this estimate and Eq. (67) that the fast electrons are lost to the wall before they reach relativistic energies when the fluctuation amplitudes $\delta B / B$ are above $0.1 \%$, unless the correlation length of the perturbations is uncharacteristically short (much shorter than the minor radius). 


\section{Re-formation of flux-surfaces}

Multiple MHD simulations [24, 28] show that the flux surfaces can re-form after the TQ, which allows the plasma to be reheated ohmically by the persistent toroidal current. However, the enhanced electric field raises a question of whether a significant fraction of the current will be overtaken by energetic electrons if flux surfaces re-form. This situation is somewhat similar to the so-called "slide-away" discharges, in which the plasma current is overtaken by RE during the current rump-up phase if the plasma density is too low.

Once the flux surfaces re-form, the transport losses become negligible and the plasma temperature should rise due to the Ohmic heating, i.e.

$$
\frac{3}{2} n \frac{d T}{d t}=\frac{j^{2}}{\sigma_{0}}\left(\frac{T_{0}}{T}\right)^{3 / 2}
$$

where the subscript "0" denotes initial values defined by Eq. (67). The inductive electric field should then decrease in time as

$$
E=\frac{j}{\sigma_{0}}\left(\frac{T_{0}}{T}\right)^{3 / 2}=\frac{j}{\sigma_{0}}\left(\frac{5}{3} \frac{j^{2}}{n T_{0} \sigma_{0}} t\right)^{-3 / 5} .
$$

To proceed with estimates, we herein consider the diffusive Dreicer leak of electrons into relativistic energies as the mechanism of RE generation (see Section V A for detailed description of this mechanism). The governing parameter in this case is the ratio of the inductive field to the Dreicer field $E_{D}$ defined in Section $V$ by Eq. $(79)$. This ratio decreases in time, because

$$
\frac{E}{E_{D}}=\frac{j}{\sigma_{0}} \frac{T_{0}}{E_{c} m c^{2}}\left(\frac{T_{0}}{T}\right)^{1 / 2} .
$$

The RE seed generated before the field drops back to the Connor-Hastie critical value (Eq. (78) ) can be estimated as a time integral of the Dreicer source (Eq. (83)). Note that the Dreicer flux is calculated for a steady state whereas the electron temperature is actually time-dependent. By using the Dreicer source in a heated plasma, we obtain an upper limit for the RE seed.

We note that the Dreicer source acts predominantly at the beginning of the re-heating process. In particular, in the ITER case, for $T=T_{0}, E_{c}=0.1 \mathrm{~V} / \mathrm{m}$ and $j=1 \mathrm{MA} / \mathrm{m}^{2}$

$$
\frac{E}{E_{D}} \approx 0.02\left(T_{0}[e V]\right)^{-1 / 2}
$$

This estimate $\left(\frac{E}{E_{D}}<0.02\right)$ guaranties the ineffectiveness of the Dreicer source in the reheating scenario, as explained further in Section $\mathrm{VA}$. 
We next consider the possibility of a runaway avalanche during the re-heating process. To do that, we integrate the Rosenbluth-Putvinski expression for the avalanche growth rate Eq. (92) over the time interval of the electric field decay. The upper integration limit corresponds to the time at which the electric field drops to the Connor-Hastie level. The avalanche gain then becomes

$$
n_{r e}=n_{r e}(0) \exp \left(\frac{3}{4 \ln \Lambda}\left(\frac{n e c}{3 \sqrt{2 \pi} j}\right)^{1 / 3}\right) .
$$

For ITER conditions, the exponent is of order unity, which precludes significant multiplication of the seed runaway population.

We, therefore, conclude that magnetic perturbations alone are not likely to produce a significant runaway population in tokamak disruptions. This is because fast electrons diffuse faster than slow electrons in the presence of such perturbations (see Eq. (68)) and because Ohmic reheating is very effective. Note, however, that some fraction of the hot population may survive the stochastic phase of the disruption without being lost. Those are the magnetically trapped electrons or electrons residing in magnetic islands. Unfortunately, there is no robust estimate for the latter. The situation changes drastically when impurities are responsible for the thermal quench. In this case, the fast electrons have a longer lifetime than the slow ones, which gives rise to the so-called "hot-tail" mechanism of runaway generation.

\section{Impurities}

In the opposite limit of the significant impurity presence and negligible perturbations, hot electrons are cooled down "on impurities". They lose energy in collisions with the initially cold electrons stripped from impurities. As shown in Ref. [89], such cold population forms when there is a sufficiently large impurity content. This is because both the ionization rate and the cold-cold collisional frequency are typically faster than the rate of collisions between the hot and cold electrons. In any case, the timescale for the temperature equilibration between the hot and the cold populations is related to the hot electrons collisionality $\left(\tau_{e e}\right)$ and is typically much shorter than the subsequent radiative energy loss timescale.

The evolution of the bulk plasma temperature is governed by the power balance equation

$$
\frac{\partial\left(W_{t h}+W_{i}(T)\right)}{\partial t}=P_{s}(F)+\frac{j_{c}^{2}}{\sigma_{c o l d}}-n_{i m p} n_{c o l d} L(T)
$$


where $W_{t h}=\frac{3}{2} n_{\text {cold }} T, W_{i}$ are the ionization losses, $n_{\text {cold }}=n_{\text {hot }}^{0}-n_{\text {hot }}+n_{\text {imp }} Z(T)$. $P_{s}$ is the stopping power released by the hot population via Coulomb collisions, $L(T)$ is the cooling coefficient [90] $]^{3} \sigma_{\text {cold }}$ is the Spitzer conductivity Eq. (62) and $j_{c}$ is the Ohmic current, which stays constant on the TQ timescale in the absence of runaways.

Figure 10 shows a typical evolution of the cold bulk temperature for the runaway-free case (taken from the simulations of [89]). The initial quick rise of the temperature is associated with a fast energy transfer from the hot pre-quench population (the first term on the right hand side of Eq. (75) ) and the extended subsequent temperature drop is due to radiative energy losses (the last term on the right hand side of Eq. (75p). The latter process is typically much longer and therefore defines the length of the TQ. However, as will be discussed further, under some conditions the radiation power may prevail over the heat exchange between the hot and cold electrons, which leads to extremely short TQs.

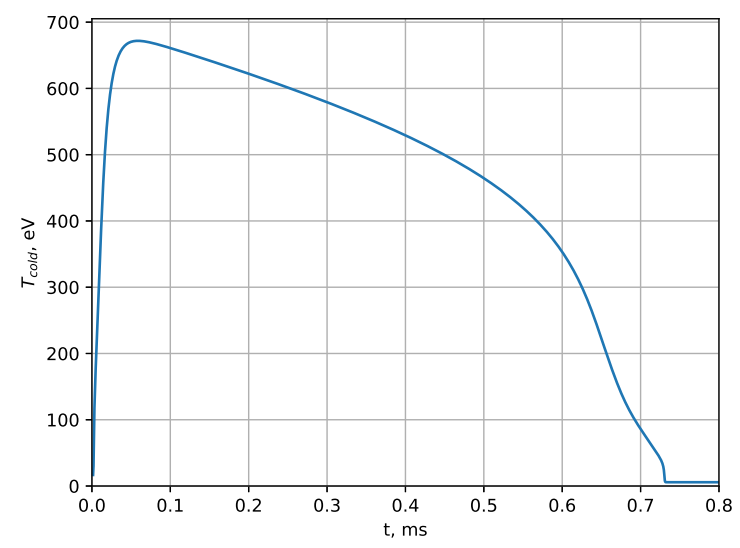

FIG. 10. Evolution of the cold bulk plasma temperature during the TQ for pre-quench plasma temperature of $5 \mathrm{keV}$, density of $10^{20} \mathrm{~m}^{-3}$ and injected Argon density of $0.3 \cdot 10^{20} \mathrm{~m}^{-3}$.

When the ionization and radiation losses can be neglected on the timescale of the initial temperature equilibration, the temperature after such "dilution" is

$$
T_{d i l} \approx \frac{n_{h o t}^{0} T_{0}}{n_{h o t}^{0}+n_{i m p} Z\left(T_{d i l}\right)},
$$

where $Z\left(T_{d i l}\right)$ denotes impurity ionization level.

\footnotetext{
${ }^{3}$ Note that the timescale of plasma cooling is longer than the relaxation time to ionization equilibrium in the plasma, which enables characterization of the radiative losses by the stationary cooling coefficient $L(T)$.
} 

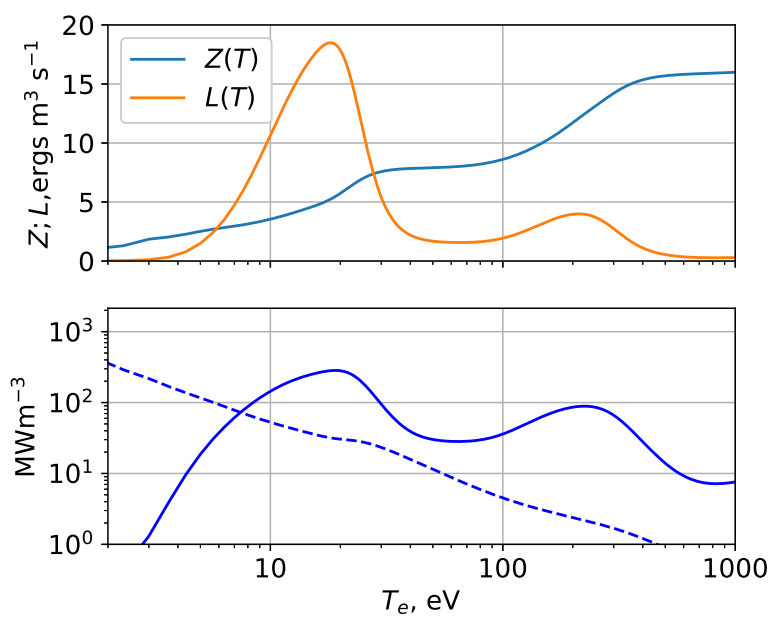

FIG. 11. Top panel: Temperature dependence of the mean ionization level $(Z)$ and radiative efficiency $(L)$ for Argon according to [90. Bottom panel: Line radiation power (solid) and ohmic heating power (dashed) for Ohmic current of $j_{0}=1 \mathrm{MA} / \mathrm{m}^{2}$, Deuterium density of $10^{20} \mathrm{~m}^{-3}$ and argon density of $10^{19} \mathrm{~m}^{-3}$.

Figure 11 presents the temperature dependencies of the mean ionization level $(Z)$ and radiative efficiency $(L)$ for Argon, as an example. The lower panel of Fig. 11 compares the Ohmic heating and line radiation temperature dependencies for a given impurity density. The radiated power is notably non-monotonic. Typically, the Ohmic heating appears to be much weaker than the line radiation when impurities are injected intentionally until the temperature drops to a few eV levels, where the two terms on the right-hand side of Eq. (75) equalize.

The peak in the radiation efficiency at moderate temperatures $\left(T_{\text {rad }}^{\max } \approx 20 \mathrm{eV}\right.$ for Argon, $\approx 40 \mathrm{eV}$ for Neon, $\approx 8 \mathrm{eV}$ for Carbon and Helium) leads to the other feasible TQ scenario. The collisional energy transfer rate from the hot pre-quench population to the cold population reduces for higher pre-quench temperature, i.e. the heating power $P_{s} \approx \frac{n_{h o t}^{0} T_{0}}{\tau_{e e}} \sim \frac{1}{\sqrt{T_{0}}}$. As a result, the radiation peak can be higher than the power deposition $\left(P_{s}\right)$ from the hot pre-quench particles at large impurity quantities. Then the cold population will remain at $T<T_{\text {rad }}^{\max }$ efficiently radiating all the transferred energy. The $T_{\text {dil }}$ will not be achieved in such a case and all plasma kinetic energy will be radiated on a very short collisional $\left(\tau_{e e}\right)$ timescale. 
The TQ duration $\left(\tau_{T Q}\right)$ itself is an important target parameter of the DMS, as elaborated in Section XI. It defines the timescale of the power deposition to the plasma facing components. Very short TQ are not desirable due to possible damage.

The TQ duration $\left(\tau_{T Q}\right)$ is shown in Fig. 12 for pre-quench deuterium density of $10^{20} \mathrm{~m}^{-3}$ and various Argon densities and pre-quench temperatures (as calculated in Ref. [89]). The horizontal axis represents radial variation of the temperature in ITER. Figure 12 shows that a very strong variation of the TQ timescales should be expected for a uniform impurity density deposition. The non-monotonicity of the TQ duration at large Argon densities $\left(>0.8 \cdot 10^{20} \mathrm{~m}^{-3}\right)$ is related to the issue of the heating power $(P)$ being lower than the $T_{\text {rad }}^{\max }$ radiation.

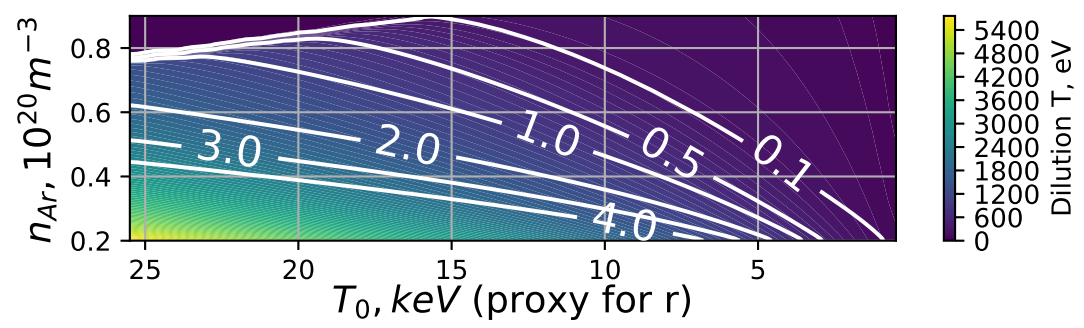

FIG. 12. Duration of the thermal quench $\left(\tau_{T Q}\right)$ (white contours in ms) for a range of initial electron temperatures $T_{0}$ and injected Argon densities. Color coded is the bulk plasma temperature after a quick dilution phase of the TQ $\left(T_{d i l}\right)$.

The non-uniformity of the impurity gas cloud can increase the radiation significantly for two reasons. First, the radiated power scales as $n_{\text {imp }} n_{\text {cold }}$ (see Eq. (75)). An initially small gas cloud should, therefore, radiate more efficiently than the same amount of uniformly distributed gas. Second, the electrons cool down adiabatically as the gas cloud expands along the field line. As demonstrated in [91], the hot electrons transfer a large fraction of their energy into the kinetic energy of impurity ions via ambipolar expansion. These two important factors have not yet been considered in TQ modeling, nor in the RE seed formation studies.

A number of studies address the RE generation during TQ [54, 89, 92 96]. Following Refs. [54, 92] the mechanism of REs formation in quickly cooled plasmas is known as the "hot tail" mechanism. 


\section{PRIMARY RUNAWAY ELECTRONS}

A plasma is a conducting medium with a distinctive feature that the rate of Coulomb collisions decreases with increasing energy. Consequently, the frictional drag force for a supra-thermal electron decreases with electron speed $v$ as $1 / v^{2}$. For an ultra-relativistic electron, this force asymptotes to

$$
\frac{4 \pi e^{4} n \ln \Lambda}{m c^{2}}
$$

where $n$ is the electron density, $m$ the electron mass, $e$ the electron charge, $c$ the speed of light, and $\ln \lambda$ the Coulomb logarithm. Application of an electric field $E$ in excess of

$$
E_{C H}=\frac{4 \pi e^{3} n \ln \Lambda}{m c^{2}}
$$

will evidently produce a runaway effect [2, 40], an unimpeded acceleration of electrons with $v>c \sqrt{E_{C} / E}$. Only a small fraction of the entire electron population falls immediately into the runaway energy range when the electric field is less than the Dreicer field,

$$
E_{D}=\frac{4 \pi e^{3} n \ln \Lambda}{T}
$$

but the runaway population tends to grow in time, because Coulomb collisions between the bulk electrons bring new particles into the runaway regime [97. The supply of new particles from the bulk itself is a diffusive process, because of the dominance of small-angle collisions within the bulk. The number of runaway electrons would grow linearly in time as a result of that. A further growth mechanism of the runaway population is via knock-on collisions between the existing runaway electrons and the bulk. The rate of the knock-on collisions does not involve the large Coulomb logarithm and is therefore relatively low, but this mechanism can easily prevail, because it gives rise to an exponential (rather than linear) avalanche-type multiplication of the runaway population.

\section{A. Dreicer source}

A tempting way to estimate the runaway production rate, $d n_{r e} / d t$, via a diffusive (Dreicer)

mechanism would be to take the density of the Maxwellian tail with $v>c \sqrt{E_{C} / E}$ and say that all these electrons become runaways within one collision time, which gives

$$
\frac{d}{d t} n_{r e} \sim \frac{e E}{\sqrt{m T}} n \exp \left(-\frac{E_{D}}{2 E}\right) .
$$


Although this simplistic estimate reflects the exponential weakness of the runaway source at $E<<E_{D}$, it is apparently too crude, because the actual distribution function of the suprathermal electrons differs considerably from the Maxwellian. This difference is of the order of unity at $v \sim \sqrt{T / m}\left(E / E_{D}\right)^{1 / 4}$, as one can see from comparing the term $(e / m)(\mathbf{E} \cdot \partial f / \partial \mathbf{v})$ in the electron kinetic equation to the collisional term. The latter can be roughly estimated as $\nu \delta f$, where $\nu \sim \frac{4 \pi e^{4} n \ln \lambda}{m^{2} v^{3}}$ is the collision frequency and $\delta f$ is the deviation of the distribution function from the Maxwellian $f$.

The need for an accurate description of the electron distribution at $v \sim \sqrt{T / m}\left(E_{D} / E\right)^{1 / 4}$ and up to the runaway velocities $v \sim \sqrt{T / m}\left(E_{D} / E\right)^{1 / 2}=c\left(E_{C} / E\right)^{1 / 2}$ was already recognized by Dreicer who used an artificial spherically symmetric model in his analysis, but prudently indicated the lack of rigor in such an approach. A subsequent work [39] has shown that the exponential factor in Eq. 80 is actually $-\frac{E_{D}}{4 E}-\left(2 \frac{E_{D}}{E}\right)^{1 / 2}$ rather than $-\frac{E_{D}}{2 E}$. Yet, it has not been precise with regard to the pre-exponential factor [98]. The preexponential factor has then been determined correctly in Ref. [99]. The resulting accurate expression for the Dreicer source in the non-relativistic limit $\left(E_{D}>>E>>E_{C}\right)$ is

$$
\frac{d}{d t} n_{r e}=C n \frac{4 \pi e^{4} n \ln \Lambda}{T \sqrt{m T}}\left(\frac{E}{E_{D}}\right)^{-3 / 8} \exp \left[-\frac{E_{D}}{4 E}-\left(2 \frac{E_{D}}{E}\right)^{1 / 2}\right],
$$

where $C$ is an order of unity constant. Connor and Hastie have extended the approach of Ref. 99] to take into account relativistic effects and the effective charge $Z$ of the plasma ions [40] (a similar independent study has also been published in Ref. [100]). Their work generalizes 81 to

$$
\frac{d}{d t} n_{r e}=C n \frac{4 \pi e^{4} n \ln \Lambda}{T \sqrt{m T}}\left(\frac{E}{E_{D}}\right)^{-h(\alpha, Z)} \exp \left[-\lambda(\alpha) \frac{E_{D}}{4 E}-\left(2 \frac{E_{D}}{E}\right)^{1 / 2} \gamma(\alpha, Z)\right],
$$

where

$$
\begin{aligned}
& \alpha \equiv E / E_{C}, \\
& h(\alpha, Z)=\frac{1}{16(\alpha-1)}\left[\alpha(Z+1)-Z+7+2 \sqrt{\frac{\alpha}{\alpha-1}}(1+Z)(\alpha-2)\right], \\
& \lambda(\alpha)=8 \alpha\left[\alpha-\frac{1}{2}-\sqrt{\alpha(\alpha-1)}\right], \\
& \gamma(\alpha, Z)=\sqrt{\frac{(Z+1) \alpha^{2}}{8(\alpha-1)}}\left[\frac{\pi}{2}-\arcsin \left(1-\frac{2}{\alpha}\right)\right] .
\end{aligned}
$$

Reference [40] also gives an excellent, instructive description of the preceding theoretical work. In particular, they correctly point out that "discussions of the pre-exponential factor 
are essentially of mathematical rather than practical significance, because the differences obtained by various authors are not large and the approximations used for the collisional term can introduce comparable errors".

The runaway flow rate defined by Eq. 82 falls steeply towards small $\frac{E}{E_{D}}$. As pointed out in Ref. [4], the electric field needs to exceed $\frac{E}{E_{D}} \sim 0.02$ to created a nonnegligible runaway population within the limited experimental time. In particular, the presence of such a threshold implies insignificance of the Dreicer generation mechanism in ITER post-disruption conditions [89, 101]. This mechanism of runaway generation is, however, competitive in many modern tokamaks.

Calculation of the Dreicer source in a tokamak should generally involve a distinction between trapped and passing electrons. As found numerically in Ref. [37], this reduces the Dreicer source by a factor $(1-0.5 \sqrt{2 \epsilon})$, where $\epsilon$ is the inverse aspect ratio. Reference [37] provides the following explanation for such reduction: "The formula can be understood in a simple way, by noting that the approximate fraction of trapped electrons (which are not affected by the electric field) is given by $\sqrt{2 \epsilon}$, and that half of these trapped electrons would have been accelerated in the electric field direction in the absence of trapping." .

A more recent work [102] reports a much stronger reduction with a numerical factor 1.2 rather than 0.5 , but it does not offer any comments on such disagreement with the previous results. Incidentally, Ref. [102] also contains a problematic conclusion (see Section VI]below) that the effect of toroidicity on the runaway avalanche growth is much stronger than that predicted by analytical theory and observed in independent numerical simulations.

Equation 82 refers to fully ionized plasmas. In the presence of partially ionized impurities, the effective charge $Z$ becomes energy-dependent, and drag from the bound electrons needs to be taken into account [103]. Reference [103] addresses this aspect numerically based on the model discussed in Section IIIC. The effect is shown to be small for all values of $E / E_{D}$ when the background plasma temperature is in a few-eV range. However, the partially ionized impurities can affect the Dreicer flow significantly at higher plasma temperatures. 


\section{B. Compton effect and Tritium decay}

Two radioactivity effects can bring seed electrons into the runaway regime: $\gamma$-radiation from the activated walls and radioactive decay of Tritium in the plasma volume.

The fusion reactor walls become radioactive under the DT neutron flux, and the emitted $\mathrm{MeV}$-range $\gamma$-rays can then create a runaway electron seed via Compton scattering on bulk plasma electrons. The density $\left(n_{\text {seed }}\right)$ of the seed electrons grows in time as

$$
\frac{d n_{\text {seed }}}{d t}=n_{e} \Gamma \sigma
$$

where $\Gamma$ is the $\gamma$-ray flux, $\sigma$ is the Compton scattering cross section, and $n_{e}$ is the bulk electron density. The Compton scattering cross section (as determined by the Klein-Nishina expression [104]) is herein of order or less than the Thomson cross-section $\sigma=\frac{8 \pi}{3} r_{e}^{2}=$ $6.6 \cdot 10^{-29} \mathrm{~m}^{2}$. The $\gamma$-ray flux can be roughly estimated from above as the neutron flux to the wall, because there is no more than one $\gamma$-quant per neutron. Let $P$ be the DT fusion power in MW. Each reaction produces $17.6 \mathrm{MeV}$. We than have

$$
\Gamma \sigma\left[s^{-1}\right] \leq \frac{P[M W]}{17.6 \times 1.6 \cdot 10^{-19}} \frac{6.6 \cdot 10^{-29}}{S\left[m^{2}\right]} \approx 2.3 \cdot 10^{-11} \frac{P[M W]}{S\left[m^{2}\right]}
$$

where $S$ is the reactor wall surface. Note that the ratio of $P[M W] / S\left[m^{2}\right]$ is an order of unity quantity in ITER. A more detailed evaluation of the Compton scattering rate can be found in Ref. [101.

The tritium half-life is about 12.5 years, and the related rate of seed production is therefore

$$
\frac{1}{n_{T}} \frac{d n_{\text {seed }}}{d t} \approx 1.8 \times 10^{-9} s^{-1}
$$

where $n_{T}$ is the Tritium density. Given that $n_{T} \approx n_{e} / 2$ in fusion plasmas, we find that the decay of Tritium is generally a faster mechanism for seed production than Compton scattering. Yet, both of them appear to be less efficient than the hot-tail mechanism discussed in Section VD,

\section{Magnetic Reconnection}

Although a thermal quench is an apparent prerequisite for runaway electron production in a tokamak plasma, this is not the case in space plasmas where magnetic reconnection 
produces energetic electrons. In particular, magnetic reconnection plays a decisive role in solar flares [105]. There are three general physics ideas regarding particle acceleration during reconnection events: (1) acceleration by the DC electric field at special locations where two opposite magnetic fluxes are forced into each other, i.e. near the $\mathrm{X}$ points of the magnetic islands; (2) stochastic acceleration of resonant particles that interact with various linear waves, such as whistlers or Alfven waves; (3) particle acceleration at the shock fronts. While these ideas are conceptually clear, their details are less than straightforward. A conclusive theory of energetic particle generation during magnetic reconnection still remains to be developed. An overview of the physics principles and current status of this work can be found in Refs. [105] and [62]. The relevance of space plasmas observations and analysis to RE generation in tokamaks is not apparent at this point. The presence of strong toroidal magnetic field in tokamaks, unlike in space plasma, requires acceleration to be aligned with the toroidal field. A recent review of magnetic reconnection in a laboratory plasma can be found in [106].

Unlike in space plasmas, evidence of energetic particle generation during magnetic reconnection in tokamaks is relatively scarce (we herein refer to the cases in which there is no thermal quench). One of such rare examples is the presence of suprathermal electrons (20-100 keV) in the sawtooth crashes at the T-10 tokamak [107]. These experiments show that energetic electrons appear around the islands $\mathrm{X}$ points during the crash and prior to the temperature drop in the density limit disruption. More recently, energetic electrons were observed on EAST during forced reconnection caused by externally applied non-axisymmetric magnetic perturbations [108]. There are also observations of energetic ions during magnetic reconnection, such as in MAST during internal events [109] and merging-compression plasma start-up [110].

Implications of fast magnetic reconnection for ITER are discussed in Ref. [13], where a phenomenological helicity conserving mean-field Ohm's law is used in order to assess the RE fate during magnetic relaxation. In particular, Ref. [13] discusses uncertainties arising from incomplete stochastization of the magnetic surfaces during the thermal quench. 


\section{Hot-tail}

Fast delivery of gas to a magnetic surface tends to create a two-component electron distribution consisting of the original hot electrons (with density $n_{h o t}$ ) and secondary cold electrons produced via ionization of the added gas (with density $n_{c}$ ). We herein consider a radially local problem and put aside the question of how deep the gas can penetrate, especially in large devices. All hot electrons slow down and lose energy to the cold population as a result of collisional drag, but it takes longer for the higher energy electrons to slow down than for the lower energy ones because the collision frequency decreases with energy. The surviving tail of the hot Maxwellian pre-quench population is strongly susceptible to running-away, as first observed in numerical calculations of [54, 92]. Reference [93] provides the first analytic description of the high energy tail of the cooled down electron population. This description, as well as a subsequent Ref. [94], assumes that the cooling rate is much lower than the collision frequency between the hot electrons, in which case the pre-quench population remains nearly Maxwellian during the cooling down, and its deformed tail is just a small perturbation.

It is, however, more likely that the cooling rate will actually be faster than the hothot collisions when the amount of impurities is as large as considered for the disruption mitigation system. This is clearly the case when $n_{c} \gg n_{h o t}$. The self-collisions within the hot population are then subdominant to the hot-cold collisions, and the initial Maxwellian distribution of the hot electrons should, in the absence of the inductive electric field, evolve as 95

$$
F=\frac{4 c n_{h o t}^{0}}{\sqrt{\pi} v_{0}} \frac{v^{2}}{v_{0}^{2}} \exp \left[-\left(\frac{v^{3}}{v_{0}^{3}}+S\right)^{\frac{2}{3}}\right],
$$

where $v_{0} \equiv \sqrt{2 T_{0} / m}, S \equiv 3 s \frac{c^{3}}{v_{0}^{3}}$, and $v=p c / \sqrt{p^{2}+1}$ is the particle velocity. It is interesting to note that the mean energy of such distribution grows in time whereas the density decreases exponentially

$$
\frac{n_{h o t}(S)}{n_{h o t}^{0}}=\frac{2 S^{\frac{1}{3}}}{\sqrt{\pi}} \exp \left[-S^{\frac{2}{3}}\right]+1-\operatorname{erf}\left[S^{\frac{1}{3}}\right] .
$$

The strong inequality $n_{c} \gg n_{h o t}$ is difficult to satisfy at early times. This raises a question of whether self-collisions can affect the hot-tail formation significantly. It is, however, provable that the hot-hot collisions can be neglected even in the case of equal densities of the cold and hot populations. The reason is that the hot population is initially Maxwellian, and 
it remains close to Maxwellian at the early stage of cooling. The hot-hot collision integral vanishes for Maxwellian distribution, which reduces the role of such collisions significantly. More specifically, the kinetic equation with the Landau collisional integral (see Eq. 11.18 in [111) can be written as:

$$
\begin{aligned}
& \frac{\partial f}{\partial t}+\frac{1}{v^{2}} \frac{\partial}{\partial v}\left(v^{2} \frac{j_{i} v_{i}}{v}\right)=0 \\
& \frac{j_{i} v_{i}}{v}=\frac{2 \pi e^{4} \ln \Lambda}{m^{2}} \int\left[\frac{f(v)}{v^{\prime}} \frac{\partial f\left(v^{\prime}\right)}{\partial v^{\prime}}-\frac{f\left(v^{\prime}\right)}{v} \frac{\partial f(v)}{\partial v}\right] K\left(v ; v^{\prime}\right) 4 \pi v^{\prime 2} d v^{\prime},
\end{aligned}
$$

where

$$
K\left(v ; v^{\prime}\right)= \begin{cases}\frac{2}{3} \frac{v}{v^{\prime}} & \text { for } v^{\prime}>v, \\ \frac{2}{3}\left(\frac{v^{\prime}}{v}\right)^{2} & \text { for } v^{\prime}<v .\end{cases}
$$

We herein ignore weak anisotropy of the electron distribution.

Figure 13 shows snapshots of the correction to the velocity flux $\frac{j_{i} v_{i}}{v}$ due to hot-hot collisions (top panel) in comparison with the flux due to hot electron collisions with the cold Maxwellian population (bottom panel). This figure refers to the distribution function given by Eq. (87). The initial temperature of the hot population is $5 \mathrm{keV}$. Initial distributions have equal densities. We observe that hot electron collisions with the cold electrons (of the same density) dominate in the velocity space by at least an order of magnitude.

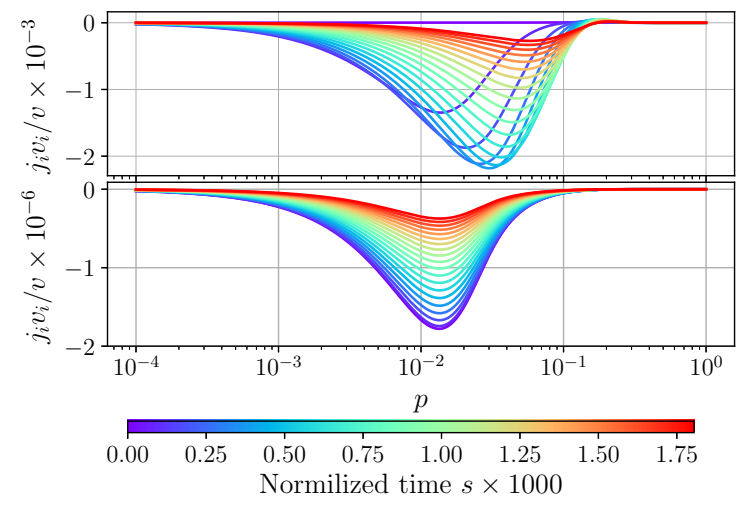

FIG. 13. Comparison of the evolution of the momentum-space flux resulting from hot-hot collisions (top panel) with the flux resulting from slowing down of the hot population on the cold background (bottom panel) at the beginning of the evolution. Note three orders of magnitude difference in scales on these panels. 
The presented evaluation of the Landau collisional integral for the cooling down distribution function confirms that it suffices to consider slowing down of the hot population on the cold bulk for accurate prediction of the hot-tail formation.

Reference [95] gives an estimate of the hot-tail seed density by counting electrons that enter the runaway regime, i.e., those above the critical velocity $v_{c}$ for runaway regime entrance:

$$
\frac{d n^{h o t-t a i l}}{d t}=\frac{\partial}{\partial t} \int_{v_{c}}^{\infty} f(t) 4 \pi\left(v^{2}-v_{c}(t)^{2}\right) d v .
$$

However, neither Ref. [95], nor some later work (such as [96]) describe the time behavior of the hot distribution function $(f)$ in Eq. $(90)$ and the inductive electric field self-consistently.

More recent work [89] provides the needed self-consistent description based on the assumption of a constant total current density

$$
j_{0}=\int e v_{\|} F d p \sin \theta d \theta+\sigma_{c o l d} E,
$$

where $j_{0}$ is the pre-quench plasma current density and $v_{\|}$is the parallel component of the electron velocity. Equation (91) together with the kinetic equation for the hot population and the power balance equation 75 form a closed set, which allows to track the evolution of the hot population distribution function during the impurity dominated TQ.

Reference [89] identifies two distinct regimes, depending on the amount of injected impurities. In the case of abundant impurity injection, the hot population carries most of the total current throughout the entire quench, because of the restrictively low conductivity of the cold background. Figures 14 and 15 illustrate the transformation of the nearly isotropic initial Maxwellian distribution into a beam-like distribution in such a case. The electric field rises in step with the slowing down of the hot population until the hot electrons reach an order-of-unity anisotropy, after which the friction force is already unable to balance the electric field drive for the current-carrying electrons. The energy of the current-carrying electrons then increases continuously whereas their density decreases and asymptotes to a finite value as these electrons form a beam-like distribution and approach relativistic energies (blue and red contours in Fig. 14a). The electric field decays in step with their acceleration, and it eventually approaches the critical level over a long time. This case represents a prompt conversion regime, in which runaway electrons carry the entire plasma current after the quench, while their energy remains in the sub-MeV range. 


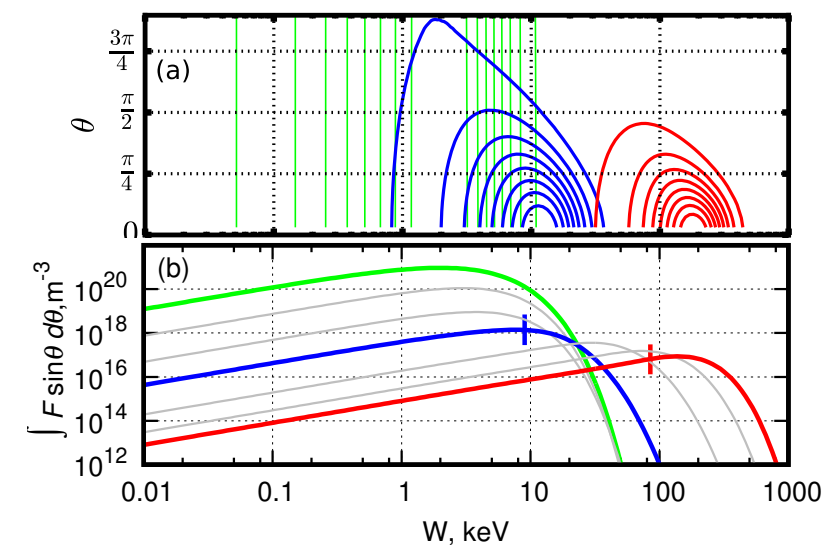

FIG. 14. Snapshots of the evolving distribution function (a) and its isotropic part (b). The colors in (a) and (b) correspond to $0 \mathrm{~ms}$ (green), $0.3 \mathrm{~ms}$ (blue) and $1.5 \mathrm{~ms}$ (red). The contours in panel (a) mark $0.9,0.8 \ldots 0.2$ of the distribution function maximum. Some intermediate profiles of the pitch-angle-averaged distribution (b) are shown in gray. The red and blue vertical strokes in (b) mark the instantaneous entrance energies to the runaway regime.

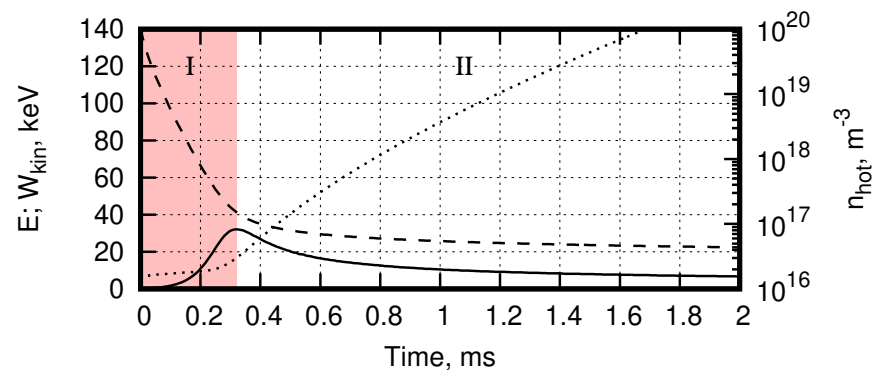

FIG. 15. Evolution of the density of the energetic electrons (dashed curve), their mean kinetic energy (dotted curve), and the inductive electric field (solid curve).

In the opposite case of moderate impurity injection, the bulk plasma conductivity remains large enough, so that the bulk electrons carry a significant part of the total current. The conducting bulk lowers the electric field initially, which facilitates the slowing down of the hot population. The cold population temperature then decreases due to radiation losses, as illustrated in Fig. 10, and the electric field quickly rises as a result. If the density of the survived hot electrons is too low to carry the total current at the time when the electric field is strong enough to prevent their slowing down, then the cold ohmic plasma has to carry most of the current. In this case, the long-lasting strong electric field accelerates the 
survived hot population to ultra-relativistic energies until new factors limit the energy gain. This scenario represents the "hot tail seed" regime. The accelerated population can then multiply subsequently via avalanche mechanism.

A closely related topic of interest is the effect of RF current drive on the hot tail formation. The RF current drive usually creates a significant non-Maxwellian tail of the electron distribution function. This may prevent any further production of the runaway electrons because the thermal quench and the resulting drop in Spitzer conductivity will not necessarily create a strong inductive electric field [112]. An assessment of runaway generation in the presence of RF current drive remains a topic for future studies. 


\section{RUNAWAY AVALANCHE IN A STRONG ELECTRIC FIELD}

This section estimates fast avalanche multiplication of the runaway population during Ohmic dissipation of the magnetic energy. The fast avalanche develops after the thermal quench when the inductive electric field becomes sufficiently strong, but all sources of the primary runaway electrons are too weak to allow the primary electrons to carry the total current. The growing current of the runaway electrons eventually dominates over the Ohmic current when the fast avalanche saturates. A subsequent slow decay of this current will be discussed in Section VIII. A distinctive feature of the fast avalanche is that the inductive electric field is much stronger than the avalanche threshold field during most of the runaway current build-up. In earlier studies [3 5], the Connor-Hastie field was deemed to represent the avalanche threshold field. It later became clear that the actual threshold is higher due to the combined effect of pitch-angle scattering and synchrotron losses (see Section VII), but the fast avalanche is insensitive to this aspect because it implies that both thresholds are very low compared the electric field of interest. It is, therefore, adequate to rely on Ref. [5] in what we discuss further in this section.

Reference [5] reduces its simplified knock-on source to a differential form and provides approximate analytical expressions for the avalanche growth rate for the idealized case of ignorable pitch-angle scattering $(Z=-1)$ and in the limit of very strong scattering $(Z \gg 1)$. These expressions follow from the corresponding solutions of the bounce averaged FokkerPlanck equation that includes acceleration by electric field, small and large angle collisional operators, but no radiative limitations on the runaway energy gain.

Based on the analytically examined limiting cases, Ref. [5] provides the following interpolated expression for the runaway avalanche growth rate:

$$
\begin{aligned}
& \frac{1}{n_{r e}} \frac{\partial n_{r e}}{\partial t}=\frac{1}{\tau \ln \Lambda} \sqrt{\frac{\pi a}{3(Z+5)}}\left(\frac{E}{E_{c}}-1\right) \\
& \times\left(1-\frac{E_{c}}{E}+\frac{4 \pi(Z+1)^{2}}{3 a(Z+5)\left(E^{2} / E_{c}^{2}+4 / a^{2}-1\right)}\right)^{-\frac{1}{2}},
\end{aligned}
$$

where $\tau \equiv m c / e E_{c}, a(\epsilon) \approx(1+1.46 \sqrt{\epsilon}+1.72 \epsilon)^{-1}$, with $\epsilon$ denoting the inverse tokamak aspect ratio.

Note that the values of the Coulomb logarithm $\ln \Lambda$ and the effective charge $Z$ in this expression need to reflect the presence of partially ionized impurities, which has not been fully detailed in Ref. [5]. In particular, Ref. [5] involves Eq. (30) for the stopping power, 
but the critical energy for runaway electrons lies in the non-relativistic range in the strong field case. The contribution from the bound electrons can be reduced significantly because of that. Reference [101] suggests correcting Eq. (92) via evaluating the Coulomb logarithm at critical energy and counting both free and bound electrons in the description of knockon collisions. This predicts more efficient avalanching than what follows from Eq. (92). However, the proposed amendments are not quite complete. They do not take into account that the critical runaway energy can be comparable to the ionization potential for many bound electrons. The knock-on collisions with energy transfer in that range may not be able to bring those bound electrons into the runaway regime because of the need to overcome the ionization potential.

Equation (92) has been compared with multiple numerical calculations of the growth rate. One of the earliest numerical benchmarks is a Monte-Carlo simulation reported in the original paper [5], where good agreement was found for various values of $Z$ and $\epsilon$ at $E>10 E_{c}$, while there was a significant $(\sim 20 \%)$ discrepancy at lower values of $E$.

Reference [54] reports good agreement between Eq. (92) and calculations with a continuous code CQL3D. This comparison involved the most general version of the knock-on source (Eq. (32)) as well as its zero-pitch-angle limit. The authors conclude that the resulting growth rate is insensitive to the form of the knock-on source used in the calculations. It should, however, be pointed out that both, the CQL3D simulations and the Monte-Carlo simulations of Ref. [5], ignore radiative losses that limit the runaway energy gain. This limitation is most pronounced at moderate electric fields, in which case the difference between the general (Eq. (32)) and the simplified sources becomes evident, as we discuss further in Section VII.

Reference [37] compares Eq. 922 with the results from a Monte-Carlo code ARENA. It reports a good agreement between the two, except for one noteworthy case of finite $\epsilon$ in the limit when the pitch angle scattering is neglected, i.e. $Z=-1$. As noted in Ref. [37], the secondary electrons produced via knock-on collisions tend to be in the trapped particle domain due to their large pitch-angles. These electrons would remain trapped in the absence of pitch-angle scattering. Consequently, they would not be accelerated by the electric field and would not contribute to the avalanche. However, the same Ref. [37] explains that the collisional de-trapping time is in fact always shorter than the avalanche growth time for realistic values of $Z$. Because of that, magnetic trapping of the secondary electrons should 
not affect the avalanche growth rate. This understanding seems to be missing in more recent work [102] that claims a much stronger impact of toroidicity on the avalanche growth rate than that predicted by Eq. (92) and found in other simulations.

Equation (92) simplifies considerably when the electric field is very strong. It then reads

$$
\frac{1}{n_{r e}} \frac{\partial n_{r e}}{\partial t} \approx \frac{1}{\sqrt{Z+5} \ln \Lambda} \frac{e E}{m c} .
$$

Given the exponential growth of the secondary electron population and continuous acceleration of those electrons, we note that the per particle energy gain is roughly $T \approx$ $m c^{2} \ln \Lambda \sqrt{(Z+5)}$ when the population doubles. The energy spectrum of the avalanching runaways is therefore insensitive to plasma parameters and takes the form [4, 5]

$$
\frac{d n_{r e}}{d \mathcal{E}} \propto \exp (-\mathcal{E} / T)
$$

Numerical simulations [5, 54] show that $T$ actually scales linearly with $Z$, i.e. $T \sim 2 m c^{2}(1+$ $Z / 6) \ln \Lambda$.

In plasma disruptions, the decay of the plasma current determines the loop voltage. Because the avalanche growth rate and the Ohmic losses are both proportional to the toroidal electric field, the saturated runaway current turns out to be insensitive to the evolution of plasma resistivity or the duration of the avalanche. This gives a simple relation between the seed runaway current and the avalanche-produced current when the radial profile effects are not considered.

The inductive electric field $E$ in the toroidal plasma volume can be estimated as

$$
E=-\frac{L}{2 \pi R} \frac{\partial I}{\partial t}
$$

where $I$ is the total toroidal current, $L=2 \pi R l_{i} / c^{2}$ is the inductance that characterizes the poloidal magnetic flux in the plasma, $R$ is the major radius, and $l_{i}$ is an order of unity factor. This inductive field is related to the Ohmic current $I-I_{r e}$ by the bulk plasma resistivity $\eta$ as

$$
E=\frac{\eta}{S}\left(I-I_{r e}\right)
$$

where $I_{r e}$ is the runaway electron current and $S$ is the plasma cross-section. We now rewrite Eq. (93) as

$$
\frac{1}{I_{r e}} \frac{\partial I_{r e}}{\partial t}=\frac{1}{\sqrt{Z+5} \ln \Lambda} \frac{e E}{m c}
$$


and combine Eqs. (95)- 97 into

$$
\frac{1}{I_{r e}} \frac{\partial I_{r e}}{\partial t}=-\frac{1}{\sqrt{Z+5} \ln \Lambda} \frac{l_{i}}{I_{A}} \frac{\partial I}{\partial t}
$$

where $I_{A}=\frac{4 \pi m c}{e \mu_{0}}=17 k A$ is the Alfvén current.

This equation can be integrated straightforwardly assuming that the internal inductance factor does not change during evolution of the current. We then obtain (see Ref. [113])

$$
\ln \frac{I_{r e}(\infty)}{I_{r e}(0)}=\frac{l_{i}}{\sqrt{Z+5} \ln \Lambda} \frac{I(0)-I_{r e}(\infty)}{I_{A}}
$$

at the end of the evolution when runaway electrons carry all the remaining current, i.e. $I_{r e}(\infty)=I(\infty)$.

Although the internal inductance may change in time, we still note that no other parameter enters Eq. (99). In particular, plasma resistivity and its evolution have no effect on the saturated RE current. Figure 16 shows how the total current and the runaway current evolve in dimensionless time units when the time is normalized to the Ohmic decay time $\tau_{p}=\frac{L S}{2 \pi R \eta}$.

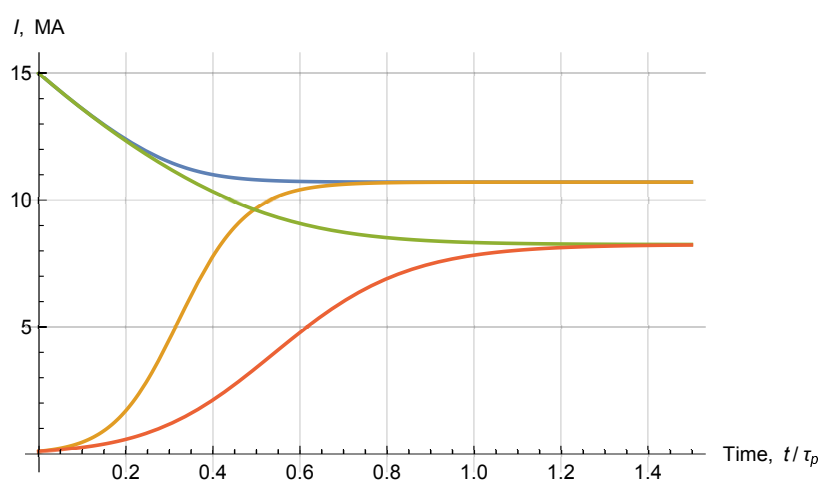

FIG. 16. Evolution of the total and runaway current during disruption. Seed RE current $I_{r e}(0)=$ $100 k A, Z=4$. Total current is indicated by blue and green curves, RE current by orange and red curves. Blue and orange curves correspond to the case with $l_{i}=1.0$, while green and red curves correspond to $l_{i}=0.6$.

The saturated value of the the RE current $I_{r e}(\infty)$ as a function of the seed RE current $I_{r e}(0)$ is shown in Fig. 17 for three cases: blue $-l_{i}=1.0, Z=4$; green $-l_{i}=0.6, Z=4$ and orange $-l_{i}=1.0, Z=12$. Note the exponential dependance of $I_{r e}(\infty)$ on $I(0)$ in Eq. (99). 


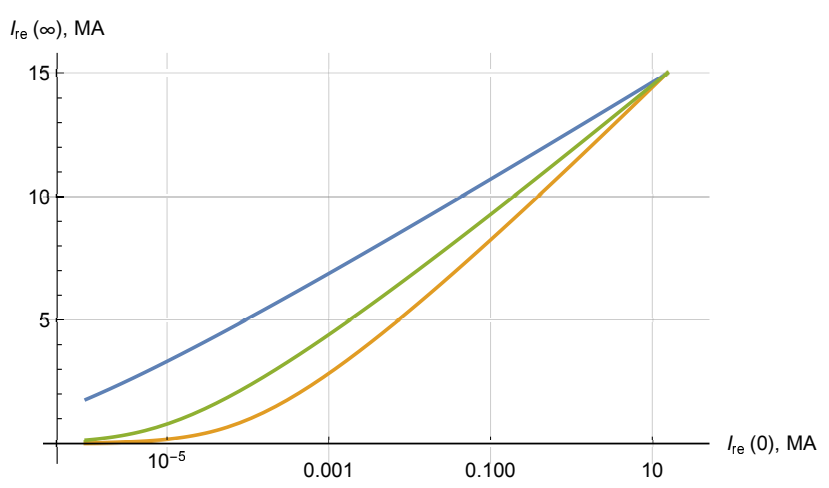

FIG. 17. Saturated value of the runaway current $I_{r e}(\infty)$ as a function of the seed RE current $I_{r e}(0)$ for $I(0)=15 M A$ and three combinations of $l_{i}$ and $Z$ : blue $-l_{i}=1.0, Z=4$; green $-l_{i}=0.6$, $Z=4$ and orange $-l_{i}=1.0, Z=12$.

While amplification of the runaway current in ITER-size tokamaks is expected to be very strong, it is much smaller in 1MA-scale devices.

When realistic plasma profiles are considered, the saturated current differs from the simple estimate (99) [113, 114]. The internal inductance tends to increase during the avalanche, and the saturated runaway current is usually more peaked at the core than the pre-quench plasma current.

An example of evolving runaway current, plasma current and electric field profiles in an ITER-like scenario during the fast avalanche is shown in Fig. 18. In this simulation, the runaway seed current profile is flat, and the total seed current is $100 \mathrm{~A}$, the plasma resistivity is calculated from the temperature balance given by Eq. (75) for a flat Argon density profile. The saturated runaway current is 4 MA. A more detailed comparison between the zerodimensional and one-dimensional calculations can be found in Ref. [113.

\section{RUNAWAY KINETICS AT NEAR-THRESHOLD ELECTRIC FIELD}

When runaway electrons overtake the majority of the plasma current, the inductive electric field drops down to a certain threshold level, governed by the "effective resistivity" of the runaway beam. This regime, dubbed "runaway plateau", is of particular practical interest, because it corresponds to a long-lasting runaway current decay and necessitates runaway mitigation. The most critical characteristics of this regime are the values of the 


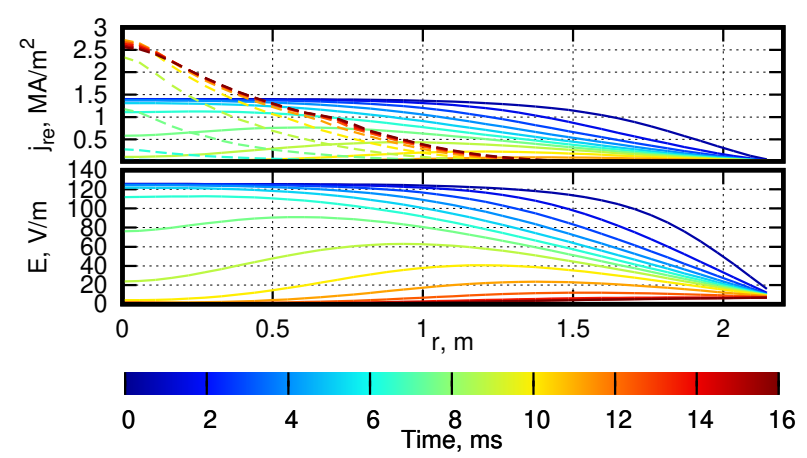

FIG. 18. Evolution of the runaway current (dashed), plasma current (solid) and electric field profiles for ITER-like parameters (data is taken from Ref. [115]).

threshold electric field and the structure of the runaway distribution function. It turns out that limitations on the runaway energy gain are essential in this regime. Because of that, the meaning of a seemingly self-explanatory term "runaway electrons" is not exactly the same as it was 60 years ago in the seminal Dreicer papers.

The initial non-relativistic studies characterize the runaway phenomenon as either unlimited acceleration of all plasma electrons in a sufficiently strong electric field or as a continuous leak of electrons from the Maxwellian tail into the runaway regime when the electric field is below the Dreicer field. A subsequent relativistic theory clarifies that the electric field has to exceed a minimum value (known as the Connor-Hastie field) to enable unlimited acceleration of the electrons. The most recent studies reveal a two-way electron flow in momentum space and establish limits on the "unlimited" acceleration. These latest findings involve a semantic conflict with the term "runaway", because the electrons do not actually run away anymore. Moreover, the accelerated electrons have a finite probability of returning to the bulk as a result of small-angle Coulomb collisions with the bulk electrons and ions. One could then think of a steady state in which a diffusive leak from the bulk (known as the Dreicer source) would balance the backflow (such situation is considered in Ref. [69]). Yet, the Dreicer source is too weak to be of interest at low temperatures, and the accelerated electrons should then have a finite lifetime as a result. This lifetime should increase with the driving electric field. The distribution function of the long-living electrons should have a distinctive shape of an eigenfunction of the electron kinetic equation. The term "long-living" implies a time-scale that is much longer than the electron drag time. 
The time-scale of knock-on collisions is also longer than the drag time, but such collisions may still be able to compete with the reverse flow and allow the long-living population to grow in an avalanche mode rather than decay. Because the avalanche growth is very slow in the near-threshold regime, we can first neglect the knock-on collisions at all and study the behaviour of the preexisting runaways. We can then use the distribution function of the preexisting runaways to understand whether they multiply or decay.

The processes that limit electron energy gain in the driving field are radial transport, bremsstrahlung, and synchrotron radiation. The role of transport can be significant in existing tokamaks, but less significant in next-generation devices, including ITER. The role of bremsstrahlung has been discussed in Refs. [63, 78, 116, 117]. Reference [116] employs Eq. (58) to estimate the cutoff energy from the acceleration-friction force balance. Reference [78] deals with a kinetic equation for the runaway distribution function established by the accelerating field and bremsstrahlung. It includes bremsstrahlung into a Boltzmann collision operator. The resultant angle-energy distribution appears to be much broader than that in the case of the mean-force approximation (Eq. (58)) for the otherwise equivalent parameters. References [63, 117] compare bremsstrahlung with synchrotron losses. They demonstrate that bremsstrahlung tends to be of secondary importance for ITER-relevant parameters. It becomes competitive only at very strong inductive electric fields that are much greater than the Connor-Hastie field. Consequently, synchrotron drag is of primary interest for large tokamaks with a strong magnetic field, including ITER. In Ref. [118] the role of the combined effect of pitch angle scattering enhanced by high-Z impurities and synchrotron radiation was emphasised.

Reference [119] describes synchrotron losses using single-particle trajectories. It shows that a set of trajectories converge to a stable focus in momentum space. This peaking is evident in Monte Carlo simulations performed in Ref. [53]. Figure 19 presents a snapshot of the numerically calculated distribution function which exhibits peaking.

A subsequent kinetic analysis in Refs. [69, 120, 121] also demonstrates the trend for fast electrons to peak in momentum space. Reference [122] emphasizes a 2-d treatment and describes a vortex structure in momentum space. The cited findings are thematically consistent: their results collectively suggest that there is an accumulation area dubbed attractor in Ref. [120] and vortex in Ref. [122], to which the runaways gather. The characteristic electron energy in this area is determined by the balance of the electric field with collisional and 


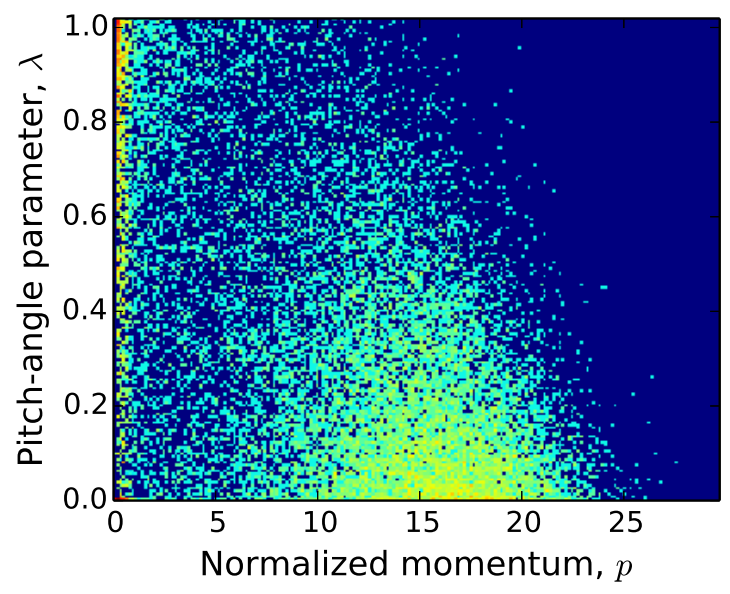

FIG. 19. Snapshot of the runaway electron distribution in momentum and pitch angle during the decay process. The pitch-angle parameter is $\lambda \equiv \sin ^{2} \theta$. Figure adopted from Ref. [53].

radiative drag. Meanwhile, the angular spread of the attractor is determined by the electric field's ability to collimate the runaways against scattering via collisions of the fast electrons with slow ions and slow electrons. The difference between the commonly assumed monotonic distribution of runaways, which is formed in the fast avalanche phase (Section VI), and the peaked distribution should apparently change the avalanche growth, the likelihood of wave excitation by the runaway beam, and the runaway mitigation assessment.

The phase space attractor comprises the main body of the fast electron distribution, but the attractor also has weakly populated wings. A description of the wings, particularly the wing that extends towards the thermal bulk, is crucial for determining the attractor lifetime because the electrons from that wing can leak into the slowing down region of momentum space. This leakage is due to elastic scattering.

The pre-existing runaways are described by the following normalized kinetic equation:

$$
\begin{aligned}
& \frac{\partial f}{\partial t}+\frac{1}{p^{2}} \frac{\partial}{\partial p} p^{2}\left[|e E| \cos \theta-\nu_{d r a g} m c\left(1+\frac{m^{2} c^{2}}{p^{2}}\right)-\frac{p \sqrt{p^{2}+m^{2} c^{2}} \sin ^{2} \theta}{m c \tau_{\text {rad }}}\right] f= \\
& \frac{1}{\sin \theta} \frac{\partial}{\partial \theta} \sin \theta\left(\frac{|e E| \sin \theta}{p} f+\frac{\nu_{e l}+\nu_{d r a g}}{2} \frac{m^{2} c^{2} \sqrt{p^{2}+m^{2} c^{2}}}{p^{3}} \frac{\partial f}{\partial \theta}+\frac{m c}{\tau_{\text {rad }}} \frac{\cos \theta \sin \theta}{\sqrt{p^{2}+m^{2} c^{2}}} f\right)
\end{aligned}
$$


where

$$
\begin{aligned}
\tau_{\text {rad }} & =\frac{3 m^{3} c^{5}}{2 e^{4} B^{2}}, \\
\nu_{\text {drag }} & =\frac{4 \pi e^{4} n_{\text {free }}}{m^{2} c^{3}}\left(\ln \Lambda_{\text {free }}+\frac{n_{\text {bound }}}{n_{\text {free }}} \ln \Lambda_{\text {bound }}\right),
\end{aligned}
$$

and $\nu_{e l}$ is defined by Eq. (40) for each ion species. The sum of $\nu_{e l}$ and $\nu_{d r a g}$ in the scattering operator includes the contribution of bulk electrons.

The quantities $\nu_{\text {drag }}$ and $\nu_{e l}$ have logarithmic dependence on particle momentum, but this weak dependence is inconsequential for practical applications, as explained in more details at the end of this Section. We, therefore, treat $\nu_{d r a g}$ and $\nu_{e l}$ as constants (evaluated a posteriori at the characteristic momentum of the sought-for distribution function) and transform Eq. (100) to the following dimensionless form

$$
\begin{aligned}
& \frac{\partial F}{\partial s}+\frac{\partial}{\partial p}\left[\hat{E} \cos \theta-1-\frac{1}{p^{2}}-\frac{p \sqrt{1+p^{2}}}{\hat{\tau}_{\text {rad }}} \sin ^{2} \theta\right] F= \\
& \frac{1}{\sin \theta} \frac{\partial}{\partial \theta} \sin \theta\left[\hat{E} \frac{\sin \theta}{p} F+\frac{\left(Z_{\text {eff }}+1\right)}{2} \frac{\sqrt{p^{2}+1}}{p^{3}} \frac{\partial F}{\partial \theta}\right. \\
& \left.+\frac{1}{\hat{\tau}_{\text {rad }}} \frac{\cos \theta \sin \theta}{\sqrt{1+p^{2}}} F\right],
\end{aligned}
$$

where

$$
\begin{aligned}
F & \equiv p^{2} f \\
s & \equiv t \nu_{d r a g}, \\
\hat{E} & \equiv|e E| /\left(m c \nu_{d r a g}\right), \\
Z_{e f f} & \equiv \nu_{e l} / \nu_{\text {drag }}, \\
\hat{\tau}_{\text {rad }} & \equiv \nu_{\text {drag }} \tau_{\text {rad }},
\end{aligned}
$$

and the particle momentum $p$ is normalized to $m c$.

The value of $\hat{\tau}_{\text {rad }}$ is typically of order 100 for ITER-like parameters. Because of that, one can ignore the shrinking of the pitch angle caused by synchrotron radiation, i.e., the last term on the second line of Eq. (102). The large value of $\hat{\tau}_{\text {rad }}$ also means that most of the long-living electrons are ultrarelativistic $(p \gg 1)$ and have small pitch-angles $(\theta \ll 1)$. These simplifications reveal important parametric features of the long-living electrons. We herein summarize the key findings; their detailed derivation can be found in Refs. [120, 123]. 
To ensure formation of the attractor with a long (much longer than the slowing down time) sustainment of the fast electrons, the driving electric field has to be greater than the sustainment threshold defined in Ref. [120] as

$$
\begin{aligned}
& \hat{E}_{0} \approx 1+\frac{\alpha \sqrt{2}}{\sqrt[6]{1+8 \alpha^{2}}}, \\
& \alpha \equiv\left(Z_{\text {eff }}+1\right) / \sqrt{\hat{\tau}_{\text {rad }}} .
\end{aligned}
$$

or, equivalently,

$$
\begin{aligned}
& E_{0} \approx \frac{m c}{|e|} \nu_{d r a g}\left(1+\frac{\alpha \sqrt{2}}{\sqrt[6]{1+8 \alpha^{2}}}\right) \\
& \alpha \equiv\left(Z_{\text {eff }}+1\right) / \sqrt{\nu_{\text {drag }} \tau_{\text {rad }}}
\end{aligned}
$$

in dimensional notations. The multiplier $\frac{m c}{|e|} \nu_{d r a g}$ equals the Connor-Hastie field (Eq. 78) when the plasma is fully ionized.

We note that the sustainment field depends on a single parameter, a combination of $Z$ and $\hat{\tau}_{\text {rad }}$. This field also limits the avalanche threshold from below. Two limiting cases are of particular interest: $\alpha \ll 1$ and $\alpha \gg 1$.

First, at $\alpha \ll 1$ the sustainment field is barely larger than $\frac{m c}{|e|} \nu_{\text {drag }}$. In this limiting case, angular distribution of the fast electrons establishes much faster than their energy distribution at $\hat{E}-1 \ll 1$, and the total distribution function can to lowest order be approximated as

$$
F=G(s ; p) \frac{A}{2 \sinh A} \exp [A \cos \theta]
$$

where

$$
A(p) \equiv \frac{2 \hat{E}}{(Z+1)} \frac{p^{2}}{\sqrt{p^{2}+1}},
$$

and $G(s ; p)$ is found by inserting Eq. 106 into Eq. (102) and integrating over all pitchangles, which eliminates the lowest order terms and gives a one-dimensional continuity equation:

$$
\frac{\partial G}{\partial s}+\frac{\partial}{\partial p} U(p) G=0
$$

with a "flow velocity" defined as

$$
\begin{aligned}
& U(p) \equiv-\left[\frac{1}{A(p)}-\frac{1}{\tanh [A(p)]}\right] \hat{E}-1-\frac{1}{p^{2}} \\
& +\frac{Z+1}{\hat{E} \hat{\tau}_{\text {rad }}} \frac{p^{2}+1}{p}\left[\frac{1}{A(p)}-\frac{1}{\tanh [A(p)]}\right]
\end{aligned}
$$


and plotted in Fig. 20 for three different values of the driving field. As shown in Fig. 20, the electrons tend to accumulate at $p_{\max }$ when the driving field exceeds $E_{0}$. They ultimately form a Gaussian peak around $p_{\max }$. The shape of this peak is determined by next-order correction to Eq. (108) that adds a diffusive term to this equation (see Ref. [123]).

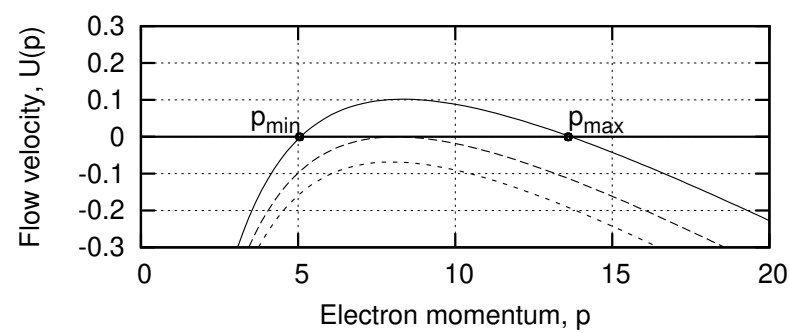

FIG. 20. A plot of flow velocity $U(p)$, given by Eq. (109). The values of the electric field are $\hat{E}>\hat{E}_{0}$ for the solid curve, $\hat{E}=\hat{E}_{0}$ for the dashed curve, and $\hat{E}<\hat{E}_{0}$ for the dotted curve.

The electrons with $p<p_{\text {min }}$ flow back into the cold core. Electrons from the wings of the Gaussian peak can also leak into the core through the lower side of the $\left[p_{\min }, p_{\max }\right]$ interval, and the lifetime of the peak depends therefore exponentially on the ratio between the width of the $\left[p_{\min }, p_{\max }\right]$ interval and the width of the peak Ref. [123]. The electric field, at which the roots $\left[p_{\min }\right.$ and $\left.p_{\max }\right]$ coincide, represents the runaway sustainment threshold $\hat{E}_{0}$ [120]. Equation 104 has been verified numerically with an adjoint Fokker-Planck equation approach in Ref. [124] and with a direct evaluation of the Fokker-Planck equation in Ref. [81. The threshold, $E_{0}$, is an important quantity in physics of runaway electrons - it determines the dissipation rate of runaway electron current in tokamaks (see Section VIII). The characteristic momentum of runaway electron population at the threshold field $\hat{E}_{0}$ for a range of $\hat{\tau}_{\text {rad }}$ and $Z_{\text {eff }}$ values is shown in Fig. 21.

In the limit of $\alpha>>1$, a large electric field (much greater than the Connor-Hastie field) is needed to sustain the fast electrons. An even larger field would be required for the avalanche onset. In this limiting case, we have $\hat{E}>>1$, and the electron drag is much weaker than the synchrotron drag for most of the sustained electrons. Consequently, a straightforward 


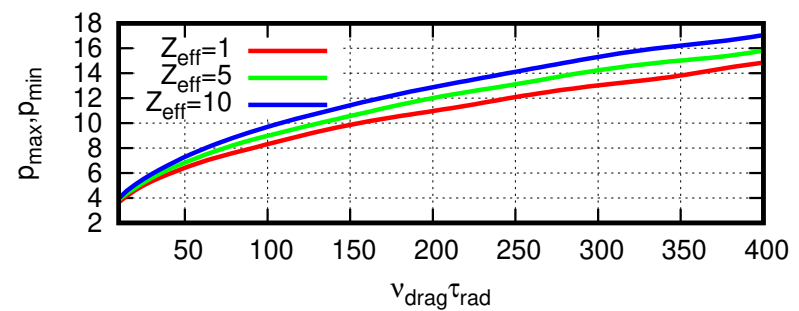

FIG. 21. Characteristic momentum of runaway electron population at the threshold field $\hat{E}_{0}$, for which $p_{\min }\left(E_{0}\right)=p_{\max }\left(E_{0}\right)$. Figure adopted from Ref. [125].

rescaling procedure,

$$
\begin{aligned}
& p=\frac{(\hat{E}-1) \hat{E} \sqrt{\hat{\tau}_{\text {rad }}}}{\alpha} P, \\
& \theta^{2}=\frac{\alpha^{2}}{(\hat{E}-1) \hat{E}^{2}} \Theta^{2}, \\
& s=\frac{(\hat{E}-1) \sqrt{\hat{\tau}_{\text {rad }}}}{\alpha} T,
\end{aligned}
$$

where the new variables are capitalized, reduces Eq. (102) to a universal form:

$$
\frac{\partial F}{\partial T}+\frac{\partial}{\partial P}\left(1-P^{2} \Theta^{2}\right) F=\frac{1}{P \Theta} \frac{\partial}{\partial \Theta} \Theta\left(\Theta F+\frac{1}{2 P} \frac{\partial}{\partial \Theta} F\right)
$$

It follows from universality of Eq. (111) that $P$ and $\Theta$ are both of order unity quantities for the sustained electrons. Consequently, Eq. (110) gives parametric scaling laws for the attractor position and width. The momentum scaling law coincides with the prediction of the single particle trajectory analysis [119] and in general agreement with the numerically verified force balance estimates of [69] and [126]. Incidentally, these scaling laws differ from what is predicted by Eq. (3.8) of Ref. [121]. They however agree better than Eq. (3.8) with the simulation results presented in Fig. 3 of Ref. [121]. The deficiency of the analytical estimate in Ref. [121 originates from unnecessary speculative assumptions involved in its derivation, whereas derivation of Eqs. (110) and (111) is free from such assumptions.

The contour plot of the slowly decaying self-similar solution of Eq. 102 for $\hat{E}=4$, $Z_{\text {eff }}=10$, and $\hat{\tau}_{\text {rad }}=5.5$ is shown in Fig. 22. Such characteristic shape of the distribution function is commonly observed in numerical simulations [69, 122, 126].

Based on rescaling (110), one might expect the lifetime of the seed electrons to be simply proportional to the driving field, which is actually not the case. The reason is that the 
lowest eigenvalue of Eq. (111) is in fact zero rather than being of order unity, because this simplified equation formally precludes any leak of electrons into the cold core. Applicability of Eq. (111) is therefore restricted to the main body of the sustained electrons, whereas calculation of the lifetime requires an asymptotic expression for the distribution function far away from its core.

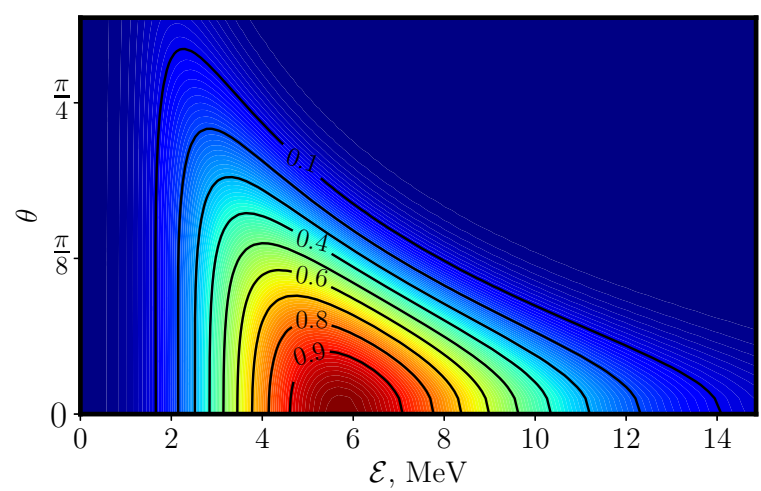

FIG. 22. Contour plot of the slowly decaying self-similar solution of Eq. 102 for $\hat{E}=4, Z_{\text {eff }}=10$, and $\hat{\tau}_{\text {rad }}=5.5$. The normalized distribution function has a steady profile peaked around $6 \mathrm{MeV}$. Figure replotted from data of Ref. [127].

The convective motion of the electrons in phase space shown in Fig. 23 connects the high-momentum and low-momentum areas at the wings of the distribution function. The scarcity of electrons in the high-momentum tail does thereby lead to their scarcity at low momenta. This feature and a refined asymptotic expression for the distribution have been used in Ref. [123] to find that the lifetime of interest will increase exponentially with the driving electric field at $\hat{E} \gg \hat{E}_{0}$, as shown in Fig. 25. An asymptotic expression for the distribution function in the ultra-relativistic tail was previously obtained in Ref. [67] as

$$
F \sim \exp \left(-2 P-P^{2} \Theta^{2}\right)
$$

This expression exhibits an exponential decay with pitch angle, but its applicability fails when $P^{2} \Theta^{2} \geq P$. A refined pitch-angle dependence at large $P^{2} \Theta^{2} \geq P$ is [123]:

$$
F \sim \exp \left(-P^{3} \Theta^{4}\right)
$$

The above described analytically tractable limiting cases of $\alpha \gg 1$ and $\alpha \ll 1$ capture 


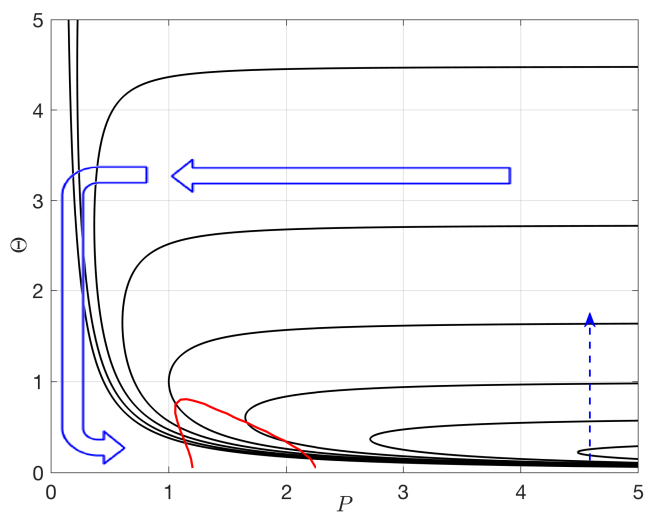

FIG. 23. Particle motion around the phase space attractor. Convective flows along solid black curves carry particles from large pitch angles and large momenta to smaller pitch angles and smaller momenta, as indicated by the blue block arrows. The dashed blue arrow represents the flux due to pitch angle diffusion. The red curve marks the phase space attractor. Figure adapted from Ref. [123].

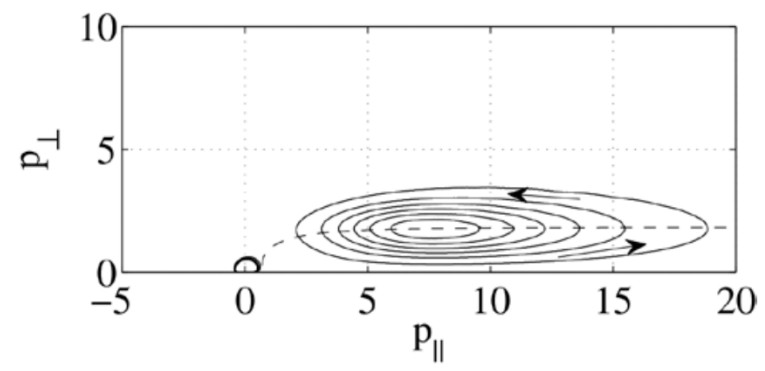

FIG. 24. Contours of the momentum space fluxes near the attractor region for a steady-state distribution. Contours are linearly distributed such that the steady-state fluxes can be inferred from the distance between two contours. Figure adopted from Ref. [69].

the essential qualitative characteristics of the seed electron population and provide useful input for experimental validation and quantitatively accurate numerical simulations.

Once the pre-existing runaway electrons have a sufficiently long lifetime, they can multiply via knock-on collisions with bulk electrons, giving rise to a runaway avalanche. The growth or decay of the population will depend on the competition between the knock-on source and 


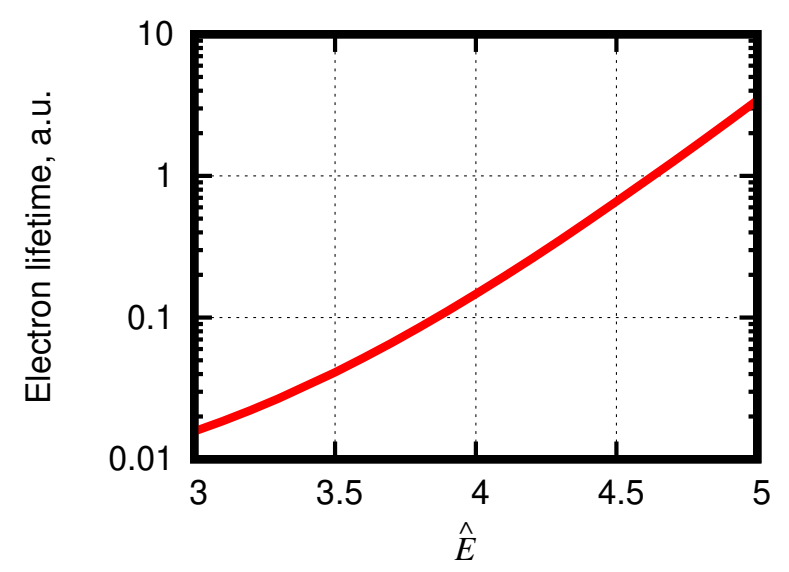

FIG. 25. Steep growth of the fast electron lifetime with the electric field. The plot is for $Z_{\text {eff }}=10$, and $\hat{\tau}_{\text {rad }}=5.5$. Figure adopted from [127].

the diffusive sink of the pre-existing population to the bulk. We note that the lifetime of the pre-existing population depends exponentially on the electric field $\hat{E}$ whereas the knock-on source has a much slower $\hat{E}$ dependence. This means that the threshold for the growth/decay of the runaway population should scale as the sustainment threshold $\hat{E}$ given by Eq. 104 and can differ from Eq. (104) only by a logarithmic factor. This conclusion is consistent with several numerical simulations [81, 128] where the Fokker-Planck equation is solved taking into account the knock-on collisions. Reference [81] uses a fully conservative knockon operator (see. Section III B). It is particularly important to ensure energy conservation for accurate calculations of the avalanche growth rate in the near-threshold regime, because large energy transfer from pre-existing electrons can knock them out of the attractor region. Such collisions act as a particle sink from the attractor, as demonstrated in Ref. [120] where the avalanche growth rate was estimated under the assumption that all pre-existing electrons are located in the attractor near $p_{\max }$. Numerical simulations in Ref. [81] do not involve such an assumption and, therefore, provide a more accurate prediction of the avalanche growth rate.

Reference [129] discusses corrections to $E_{0}$ due to momentum dependence of the logarithmic factors in Eq. (105) and due to the bremsstrahlung losses. In practice, the characteristic momentum of runaway distribution function at the threshold field $E_{0}$ varies only within a limited range (from 5 to 15) for realistic parameters (see Fig. 21). Figure 26 compares the value of the effective critical field $E_{0}$ (given by Eq. 105 for $p=5$ and $p=15$ ) with the 
results of Ref. [129] for the parameters relevant to runaway current mitigation in ITER.

For this comparison, we evaluate $\nu_{e l}$ and $\nu_{d r a g}$ by using our Eqs. (40), (27) and (29), and we replace the Coulomb logarithm $\ln \Lambda^{e e}$ in Eq. (23) of Ref. [129] with the one given by our Eq. (29). The reason for such replacement is that the relativistic Coulomb logarithm used in Ref. [127] is problematic: it depends on background plasma temperature, whereas the collisional drag force is insensitive to the background temperature for relativistic electrons (see Section III B). As seen from Fig. 26, the corrections discussed in Ref. [129] are minimal, except at high Argon densities where such corrections are predominantly due to bremsstrahlung losses.
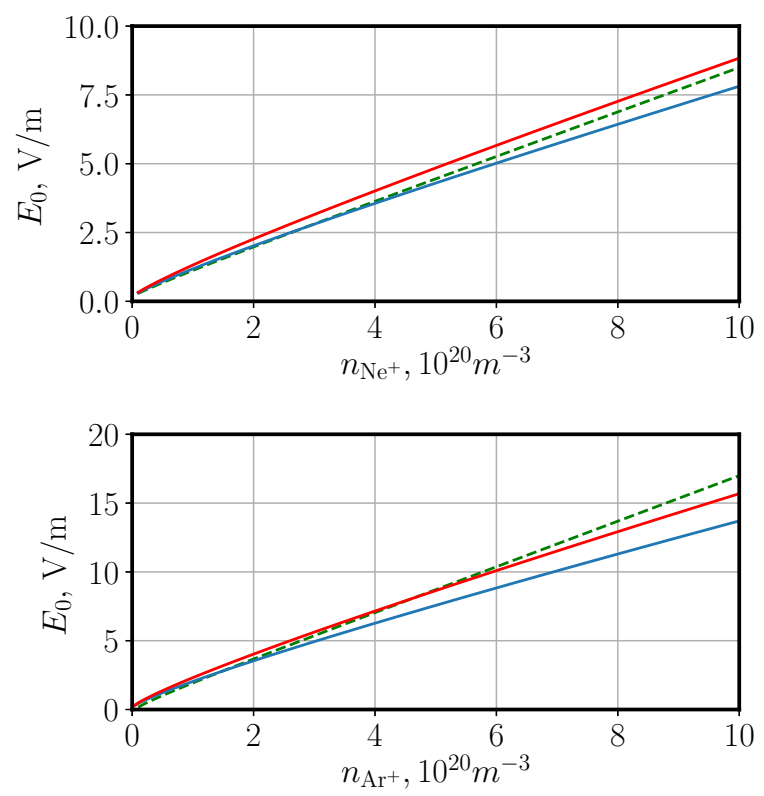

FIG. 26. Effective critical field $E_{0}$ (Eq. 105) for Deuterium plasma with $\mathrm{Ne}^{+}$(upper panel) and $\mathrm{Ar}^{+}$(lower panel) impurities. The deuterium density is $10^{20} \mathrm{~m}^{-3}$. The magnetic field is $B=5.2 \mathrm{~T}$. The blue and red curves correspond to electron momenta $p=5 m c$ and $p=15 m c$, respectively, in the logarithmic factors. The dashed curves represent Eq. (23) of Ref. [129].

\section{RUNAWAY CURRENT DECAY}

The existence of a toroidal current and the related strong poloidal magnetic field allow to view any tokamak as an inductive storage device. The stored poloidal field energy is always 
greater than the plasma thermal energy and it is much greater than the directed energy of the current-carrying particles. Consequently, the decay time of the tokamak current is considerably longer than the thermal energy confinement time. It is also much longer than the growth time of the runaway electron avalanche in any electric field that exceeds the avalanche threshold significantly. The avalanche-produced runaway current should decay in line with dissipation of the stored magnetic energy after the fast avalanche saturates. This decay can be described in terms of self-sustained marginal criticality [130], which implies that the inductive electric field stays close to the avalanche threshold within a slowly evolving current channel. Also, the runaway current should vanish at any point where the field is subcritical, because the runaway population cannot be sustained there. These two conditions specify a nonlinear "Ohms law" for the runaway current. To illustrate the marginal criticality regime, we herein adopt Eq. (104) for the threshold field and assume, in the spirit of Ref. [5], that the time-scale of the avalanche growth can be estimated as

$$
\tau_{a v} \sim \frac{m c}{e E} \sqrt{Z} \ln \Lambda
$$

Based on dimensional arguments, the knowledge of the threshold field gives an immediate estimate for the lifetime of the runaway current $I$ [130]

$$
\tau \sim \frac{I}{c^{2} E_{0}}
$$

The marginal criticality scenario implies that the ratio of $\tau / \tau_{a v}$ is large, i.e.

$$
\tau / \tau_{a v} \sim \frac{e I}{m c^{3} \sqrt{Z} \ln \Lambda} \frac{E}{E_{0}}=\frac{I}{I_{A} \sqrt{Z} \ln \Lambda} \frac{E}{E_{0}}>>1
$$

which is indeed the case when the runaway current is in a MA range.

Once combined with Ampere's law and Faraday's law in a nearly cylindrical configuration of a large aspect ratio torus, the marginal criticality condition gives a simple analytical model [130] of the slowly evolving runaway current profile. This model eliminates the avalanche time-scale from consideration and extends the analysis of Refs. [67, 131].

When geometrical factors are taken into account the estimate 115 gives the following current decay rate

$$
\frac{d I}{d t} \approx \frac{2 \pi R}{L_{i}} E_{0}=\frac{4 \pi}{l_{i} \mu_{0}} E_{0}
$$

Figure 27 shows this decay rate expected for ITER conditions in comparison with the results of the 1D current decay modelling which takes into account current profiles evolution [132] (see also Ref. [101]). 


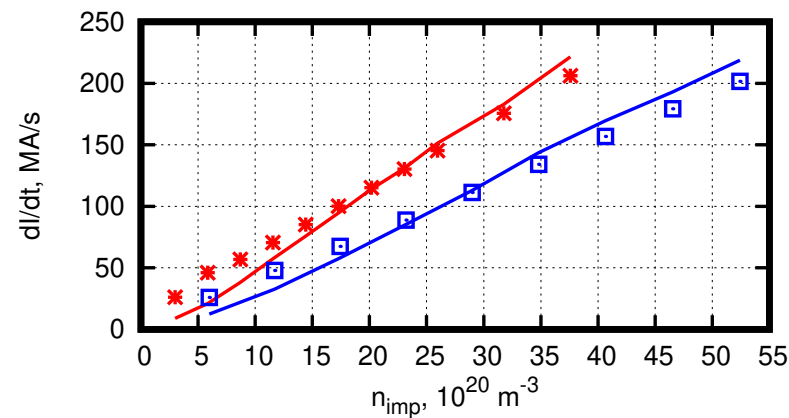

FIG. 27. The total current decay rate in ITER conditions as a function of impurity amount: Argon (red) and Neon (blue). Figure adopted from Ref. [132].

It should be pointed out that the marginal criticality scenario of the current decay implies global equilibrium and macroscopic stability of the system whereas evolution of the current profile may provoke MHD instabilities. Linear MHD-analysis of the post-disruption plasma in ITER reveals a variety of unstable MHD modes [133]. Ideal MHD instabilities do not depend on whether the bulk electrons or runaway electrons carry the current and are, therefore, the same in plasmas with and without runaway electrons, as long as the current profile is the same. Resistive instabilities are somewhat different. Their threshold is still determined by just the current profile, but their growth rate and the saturation amplitude depend on the resistivity of thermal electrons and are hence affected by the runaways [134]. Self-consistent assessment of the runaway impact on unstable MHD modes is a yet unfulfilled challenge. In particular, a radially non-uniform spatial profile of the runaway current can change in a convective way, which is essential for MHD simulations. Moreover, as commonly observed in experiments and simulations, the plasma column tends to move vertically during the current quench phase of the disruption [6]. Runaway electrons from the plasma core can strike the first wall during this motion [34]. It is predicted numerically [135], found experimentally [136], and examined in more detail in Refs. [132, 137, 138] that a significant fraction of the poloidal magnetic field energy can be converted into the runaway electron energy when the plasma touches the wall. The vertical displacement events (VDE) can change the runaway behavior significantly.

Consequently, runaway kinetics and VDEs need to be modeled self-consistently. This effort is presented in Refs. [132, 138]. 
The timescale of vertical displacement events (VDE) in tokamaks is resistive rather than Alfvénic [139, 140]. This suggests that a VDE is an adiabatically slow transition from one equilibrium state to another in a force-free way. A force-free model has been implemented in a two-dimensional code DINA [141]. A more recent code JOREK [142, 143] does not involve such simplification. It resolves the Alfvénic time-scale, and it is also three-dimensional. References [144, 145] illustrate the physical mechanism of a VDE by considering a set of inductively coupled current rings (see Fig. 28).

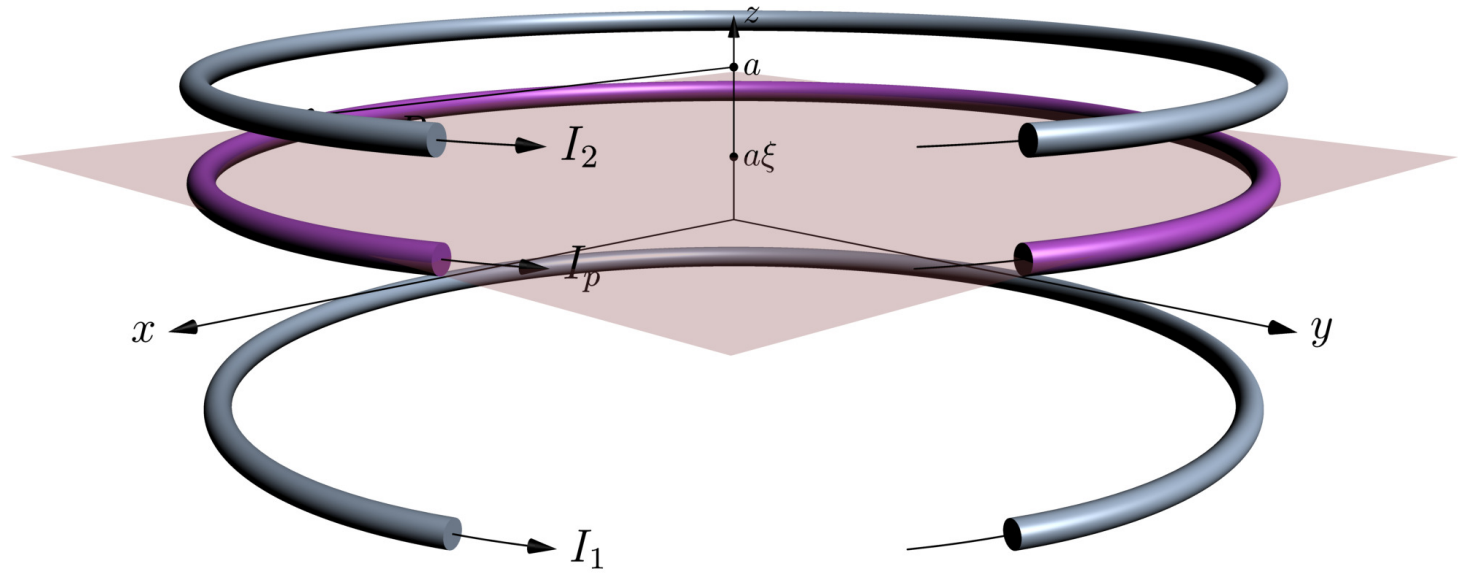

FIG. 28. Three-loop model of the toroidal plasma (middle loop) and the conducting wall (outer loops) $\xi \in[-1,1]$ is a normalized vertical displacement of the plasma from the midplane.

The three coaxial circular loops in this figure represent the plasma and the chamber wall. Reference [145] retains rapid Alfvén oscillations in such a model, whereas Ref. [144] reduces the problem to just the slow motion by using the force-free approximation. The three-loop model of Ref. [144] also includes a static external magnetic field created by two constant circular currents $I_{e}$. The corresponding circuit equations are

$$
\begin{gathered}
L_{w} \frac{d I_{1}}{d t}+L_{12} \frac{d I_{2}}{d t}+L_{w p} \frac{d}{d t}[1-\kappa \ln (1+\xi)] I_{p}=-R_{w} I_{1}, \\
L_{12} \frac{d I_{1}}{d t}+L_{w} \frac{d I_{2}}{d t}+L_{w p} \frac{d}{d t}[1-\kappa \ln (1-\xi)] I_{p}=-R_{w} I_{2}, \\
L_{w p} \frac{d}{d t}[1-\kappa \ln (1+\xi)]\left(I_{1}+I_{e}\right)+L_{w p} \frac{d}{d t}[1-\kappa \ln (1-\xi)]\left(I_{2}+I_{e}\right)+L_{p} \frac{d I_{p}}{d t}=-R_{p} I_{p},
\end{gathered}
$$

where $I_{1}$ and $I_{2}$ are the currents in the two immobile loops (wall currents), $I_{p}$ is the current in the movable loop (plasma current), $\xi$ is the normalized vertical displacement of the plasma, $\kappa=\left(\ln \left[8 R_{0} / a\right]-2\right)^{-1}$, and all the resistance and inductance coefficients are defined in [144]. 
Equations (118) together with a force-free constraint

$$
\xi=\frac{I_{1}-I_{2}}{I_{1}+I_{2}+2 I_{e}},
$$

form a closed set. Of particular interest is a limiting case of perfectly a conducting wall $\left(R_{w}=0\right)$, as it mimics the envisioned mitigated disruption scenario in ITER where the plasma current decay time is expected to be much shorter than the wall resistive time [146].

In this limiting case, Eqs. 118 and 119 reduce to an algebraic relation between $I_{p}(t)$ and $\xi(t)$, which predicts a monotonic relation between the plasma current and plasma displacement. The absolute value of $\xi$ should then increase in step with the current decay irrespective of the current carriers and current decay timeline. This adiabatically slow evolution implies that the plasma remains stable with respect to ideal MHD perturbations. It is, however, shown in [144 that the plasma column becomes unstable when the displacement reaches a certain critical value. This would apparently accelerate the VDE and make it less controllable. For ITER parameters, the critical displacement can be a substantial fraction of the minor radius.

In the presence of runaway electrons, the current $I_{p}$ in Eqs. (118), (119) becomes a sum of the bulk plasma current (characterized by Spitzer resistivity) and the runaway current, for which Ref. [144] adopted an Ohm's law proposed in Ref. [5]. Figure 29 presents a corresponding numerical solution of Eqs. (118), (119) with impurity content chosen in such a way that the current decay time was shorter than the wall resistive time. As indicated in Fig. 29, the plasma becomes unstable once the displacement passes $\xi_{c} \approx 0.5$. This ideal instability is similar to the one discussed in Ref. [147] on page 400.

It is instructive to compare the tendencies inferred from Ref. [144] with simulations performed with the DINA code for realistic plasma profiles [148]. DINA [141] describes the disruptive plasma by a magnetic field diffusion equation, Grad-Shafranov equation, transport equations, and circuit equations all solved self-consistently, but the built-in forcefree assumption does not allow DINA to identify the MHD stability boundary. JOREK does not have that limitation because it solves reduced MHD equations dynamically [143] and can, therefore, characterize both the force-free evolution and the unstable regime.

Figure 30, inferred from the ITER-relevant disruption database of DINA [149], relates the plasma vertical position $z$ to the instantaneous toroidal current $I$. In order to check the algebraic relation between the vertical displacement and the decaying current, the figure 

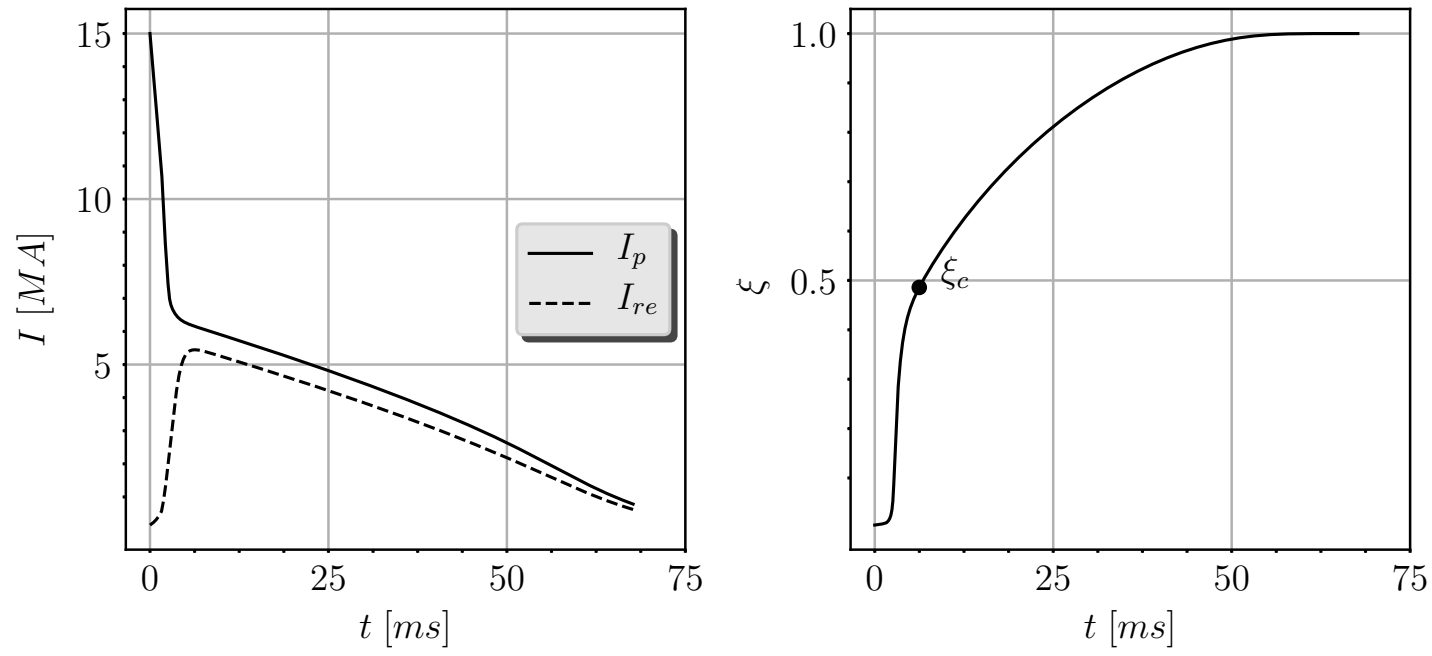

FIG. 29. Numerical solution of equations (118) and (119) with a fraction of runaway electron current. The case of the mitigated disruption for ITER plasma parameters with the massive gas injection of argon $n_{A r}=3 \cdot 10^{21} m^{-3}$. Left panel: time evolution of the plasma current $\left(I_{p}\right)$ and the runaway electron current $\left(I_{r e}\right)$. Right panel: time evolution of the plasma vertical displacement $(\xi)$.

presents disruptions with both the predominantly bulk current (solid lines) and the predominantly runaway current (dashed lines). The timescales of the current quench and the relation between $z$ and $I$ are indeed in line with the idealized model of coupled loops [144]. In particular, Fig. 30 shows that $z(I)$ has a monotonic dependence and that this dependence is universal in the ideal wall limit. 


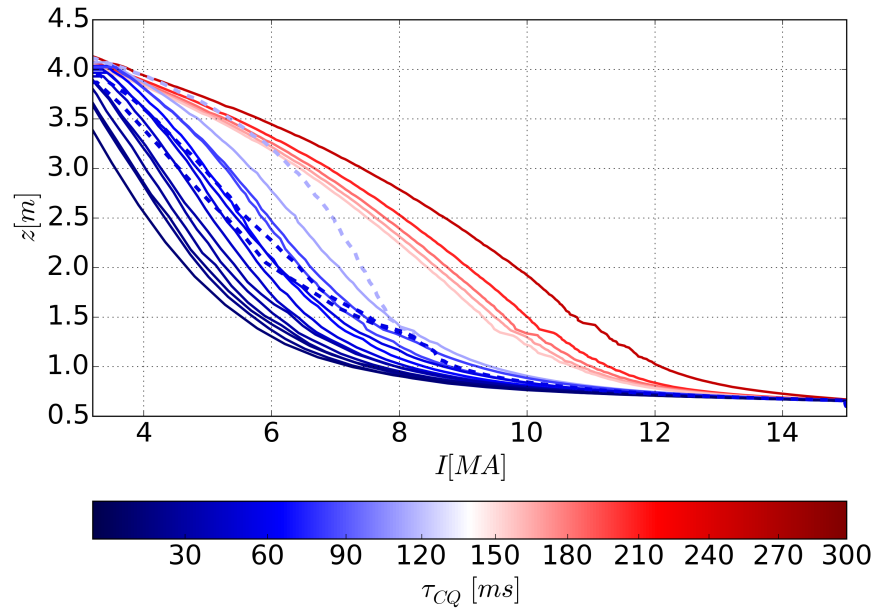

FIG. 30. DINA code results. The plasma vertical position as a function of the plasma current. Colors represent the current quench duration (red - plasma current decay time is of the same order as the wall resistive time, blue - plasma current decay time is much shorter than the wall resistive time). Figure adopted from Ref. [149]. 


\section{KINETIC INSTABILITIES OF RUNAWAY ELECTRONS}

A strongly anisotropic distribution of the runaway electrons is generally prone to high frequency and short wavelength kinetic instabilities that cause enhanced scattering of the runaway electrons. The potential benefit from enhanced scattering is a dramatic enhancement of energy losses via synchrotron radiation. The kinetic instabilities change primarily the momentum distribution rather than the spatial distribution of the fast electrons. Excitation of these instabilities can therefore be more consequential than fast electron losses to the walls.

Excitation of high frequency electron waves by runaway electrons has been observed in the early tokamaks, such as TM-3, T-6 [150, 151], TFR [152] and others, and most recently in TFTR [153] and DIII-D [154]. The early experiments have been interpreted qualitatively in Refs. [8, 155], where the observed intermittent pattern of wave saturation has been explained by the competing roles of the anomalous Doppler resonance and Cherenkov resonance. This interpretation was based on an idealized spatially local analysis. The analysis was also limited to the nonrelativistic case and it dealt exclusively with magnetized plasma waves. These limitations have motivated later studies by other authors [156-158] in an attempt to develop a more complete theory, but that effort involved controversial steps with problematic conclusions regarding instability thresholds, as explained in Ref. [159].

In what follows, we review the linear formalism used in Ref. [159] and outline a quasilinear approach to modeling runaway electrons in the presence of kinetic instabilities.

The high frequency and short wavelength oscillations are usually tractable within the WKB approximation as wave packets (quasi-particles) that have Hamiltonian equations of motion in the $(\mathbf{k}, \mathbf{r})$ phase space,

$$
\begin{aligned}
\frac{d \mathbf{r}}{d t} & =\frac{\partial}{\partial \mathbf{k}} \omega(\mathbf{k}, \mathbf{r}), \\
\frac{d \mathbf{k}}{d t} & =-\frac{\partial}{\partial \mathbf{r}} \omega(\mathbf{k}, \mathbf{r}),
\end{aligned}
$$

where the Hamiltonian is the wave frequency $\omega(\mathbf{k}, \mathbf{r})$ that is determined by the local dispersion relation. A cold plasma approximation is generally adequate for the waves of interest. The corresponding wave equation and dispersion relation have the form

$$
\left[N_{\alpha} N_{\beta}-\delta_{\alpha \beta} N^{2}+\varepsilon_{\alpha \beta}\right] E_{\beta}=0
$$




$$
\operatorname{det}\left[N_{\alpha} N_{\beta}-\delta_{\alpha \beta} N^{2}+\varepsilon_{\alpha \beta}\right]=0,
$$

where $N_{\alpha} \equiv k_{\alpha} c / \omega$ is the wave refractive index,

$$
\varepsilon_{\alpha \beta}(\omega) \equiv \varepsilon \delta_{\alpha \beta}+i g e_{\alpha \beta \gamma} b_{\gamma}+(\eta-\varepsilon) b_{\alpha} b_{\beta}
$$

is a dielectric tensor, $b_{\alpha} \equiv B_{\alpha} / B$ is the unit vector along the magnetic field, and $E_{\beta}$ is the wave polarization vector. It follows from Eq. (122) that the wave polarization vector can be written as

$$
\begin{aligned}
& \mathbf{E}=\frac{E_{x}(\mathbf{N}-\mathbf{b}(\mathbf{N} \cdot \mathbf{b}))+E_{y}[\mathbf{b} \times \mathbf{N}]}{\sqrt{N^{2}-(\mathbf{N} \cdot \mathbf{b})^{2}}}+E_{z} \mathbf{b}, \\
& E_{x}=1, \\
& E_{y}=i \frac{g}{\varepsilon-N^{2}}, \\
& E_{z}=-\frac{(\mathbf{N} \cdot \mathbf{b})}{\eta-N^{2}+(\mathbf{N} \cdot \mathbf{b})^{2}} \sqrt{N^{2}-(\mathbf{N} \cdot \mathbf{b})^{2}} .
\end{aligned}
$$

In the absence of collisions and fast particles, the dielectric tensor is Hermitian, and its components are

$$
\begin{aligned}
& \varepsilon=\varepsilon^{H} \equiv 1-\sum_{e ; i} \frac{\omega_{p}^{2}}{\omega^{2}-\omega_{c}^{2}}, \\
& g=g^{H} \equiv-\sum_{e ; i} \frac{\omega_{c}}{\omega} \frac{\omega_{p}^{2}}{\omega^{2}-\omega_{c}^{2}}, \\
& \eta=\eta^{H} \equiv 1-\sum_{e ; i} \frac{\omega_{p}^{2}}{\omega^{2}},
\end{aligned}
$$

where $\omega_{p}$ and $\omega_{c}$ are the plasma frequency and the non-relativistic cyclotron frequency, respectively. Expression (126) involves summation over particle species with $\omega_{c}$ having opposite signs for electrons and ions.

Electron-ion collisions in the bulk plasma introduce a small anti-Hermitian addition, $\varepsilon_{\alpha \beta}^{A}$, to the dielectric tensor with a resulting collisional damping of the high-frequency waves. The collisional damping rate $\Gamma_{\nu}$ can be determined via the standard perturbation technique applied to the wave equation (122), which gives the following expression for $\Gamma_{\nu}$ :

$$
\Gamma_{\nu}=-i \frac{E_{\alpha}^{*} E_{\beta} \omega^{2} \varepsilon_{\alpha \beta}^{A}}{E_{\alpha}^{*} E_{\beta} \frac{\partial}{\partial \omega} \omega^{2} \varepsilon_{\alpha \beta}^{H}} .
$$




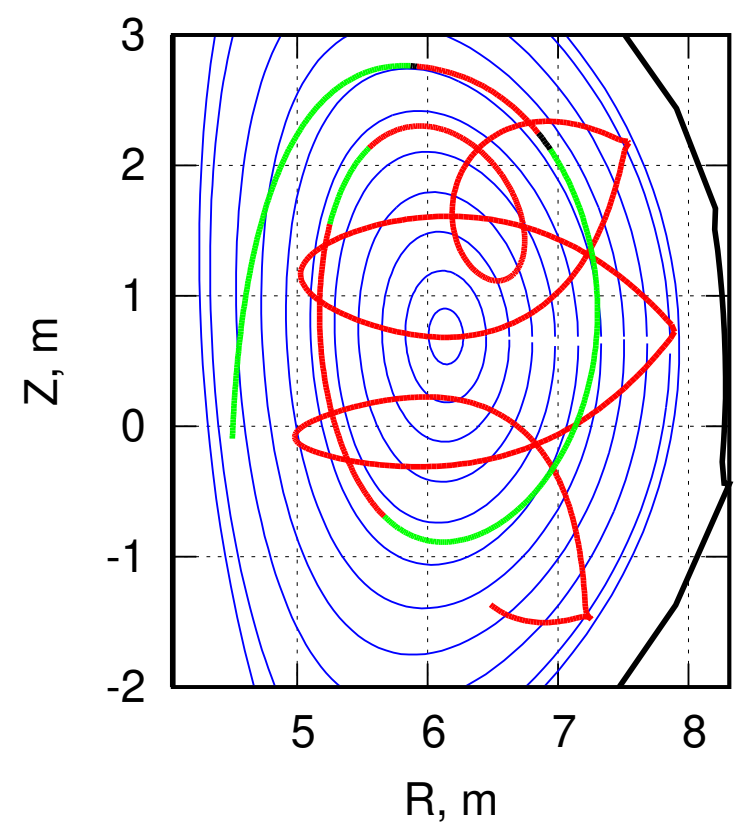

FIG. 31. An example of a wave packet trajectory. Green (light) parts of the curves - magnetized plasma branch, red (dark) part of the curve - whistler wave branch.

In particular, for high-frequency electron waves that do not involve ion motion,

$$
\Gamma_{\nu}=\nu_{e i} \frac{E_{\alpha}^{*} E_{\beta} \omega \frac{\partial}{\partial \omega} \omega\left(\varepsilon_{\alpha \beta}^{H}-\delta_{\alpha \beta}\right)}{E_{\alpha}^{*} E_{\beta} \frac{\partial}{\partial \omega} \omega^{2} \varepsilon_{\alpha \beta}^{H}},
$$

where

$$
\nu_{e i}=\frac{4 \sqrt{2 \pi} e^{4} \ln \Lambda}{3 m^{1 / 2} T_{e}^{3 / 2}} \sum_{Z} Z^{2} n_{Z}
$$

is the electron-ion collision frequency.

Equations 127), 129) correct the earlier inaccurate expressions for collisional frequency [160] and collisional damping [156 158]. The origin of those inaccuracies is explained in Section 3 of Ref. [159].

Ref. [159] also presents a ray-tracing code COIN (convective instability) that is designed to quantify the instability threshold taking into account collisional damping of the waves and spatial non-uniformity of the system for any given equilibrium configuration of the plasma and any distribution function of the runaway electrons. The code evaluates an amplification factor of the wave, $K=\int\left(\Gamma_{b}-\Gamma_{\nu}\right) d t$, by integrating the kinetic drive $\Gamma_{b}$ and the collisional damping rate $\Gamma_{\nu}$ along the wave packet trajectory. 


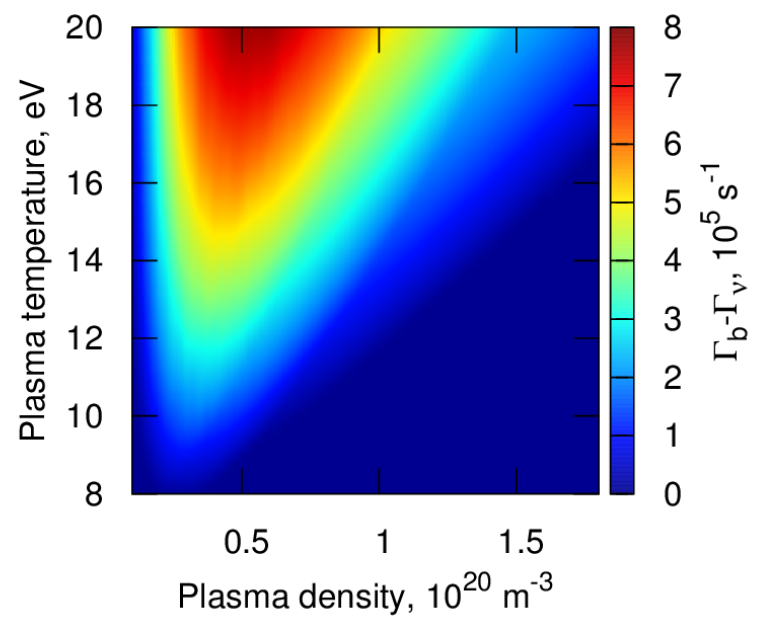

FIG. 32. Maximized growth rate for whistlers (colour coded) shows the existence of instability for a range of plasma densities and temperatures.

Radial non-uniformity of the tokamak plasma creates a cavity for whistlers and magnetized plasma waves with multiple transformations of these two modes into each other as shown in Fig. 7 of Ref. [159] and in Fig. 31. The calculated instability thresholds are consistent with previous experimental observations of the runaway-driven instability in several tokamaks (T-3, T-6, TFR, and T-10). The COIN code also specifies an instability window for ITER-relevant parameters (see Fig. 32). The resulting instability thresholds have recently been confirmed in Ref. [161] with the use of a ray tracing code GENRAY [162, 163]. Collisional damping effectively precludes excitation of kinetic instabilities by runaway electrons for post-disruption ITER parameters with electron temperature less than about $20 \mathrm{eV}$. Such instability can however develop at higher temperatures and lower plasma densities, which is the case in recent DIII-D experiments [154].

For a given shape of the runaway distribution function, the instability drive scales linearly with the runaway current density. The marginal stability argument then suggests that reduction of the collisional damping would lower the runaway current via enhanced electron scattering by the marginally sustained waves. The level of such waves would then be proportional to the driving electric field. This scenario implies that the kinetic instability actually hinders the exponential growth of the runaway current, which calls for nonlinear modeling of the instability consequences. 
Recent quasi-linear simulations within an idealized spatially uniform model [164] exhibit a strong effect of the excited waves on the RE electron spectrum. The authors also conclude that the instability can increase the avalanche threshold field significantly above the ConnorHastie value, which is qualitatively consistent with DIII-D observations.

It would be important to use such simulations to test the validity of the marginal stability concept explicitly, but that requires a dedicated analysis of the quasi-stationary electron distribution function together with the spectrum of waves that maintains a momentum balance between the driving electric field, collisional processes, and quasilinear diffusion. Besides, the quasilinear diffusion operator used in Ref. [164] is inaccurate and needs to be corrected to the flux divergence form to ensure particle conservation.

Another important aspect is that the quasi-linear code developed in Ref. [164] needs to be generalized to cover the role of plasma non-uniformity, which is essential for accurate quantitative interpretation of the current experiments and predictions for ITER. Such a generalization is feasible within a WKB approximation in the spirit of a GENRAY/CQL3D code bundle [162, 165] or C3PO/LUKE [166]. The two effects of non-uniformity are: (1) violation of the wave-particle resonance and (2) multiple bounces of the wave packets within the plasma channel. The corresponding formalism is well established, but its numerical implementation is still challenging, because the required generalization increases dimensionality of the problem. Such quasi-linear modeling did not look affordable in the past, but present-day computers make it feasible.

Quasi-linear diffusion of magnetized fast electrons in momentum space results from stimulated emission and absorption of waves via wave-particle resonances. This classical process can be conveniently described by the balance equations for particles and waves (quasiparticles) in a quantum process of their resonant interaction. The wave growth rate and the particle diffusion coefficient are then related to the probability for an electron to emit a wave with a frequency $\omega$ when the electron changes its parallel momentum from $p_{\|}$to $p_{\|}-\hbar k_{\|}$ and the quantum number of its gyro-motion by an integer $l$. The corresponding classical resonance condition,

$$
\omega(\mathbf{k}, \mathbf{r})-k_{\|} v \cos \theta-l \omega_{c} / \gamma=0
$$

reflects the energy-momentum conservation laws in this process, and the emission probability is therefore proportional to $\delta\left(\omega(\mathbf{k}, \mathbf{r})-k_{\|} v \cos \theta-l \omega_{c} / \gamma\right)$, where $v=p / m \gamma$ is the particle velocity, and $\gamma$ is the relativistic factor. The emission probability has an additional 
positive factor $U_{l}(\mathbf{p} ; \mathbf{k})$ in front of the delta-function. This factor is a function of the particle momentum and the wave-vector, and it is specific to the type of waves under consideration.

The resulting coupled kinetic equations for particles and waves have the following structure

$$
\begin{aligned}
\frac{d f}{d t} & =\left[\frac{1}{p^{2}} \frac{\partial}{\partial p} p^{2}\left(D_{p p} \frac{\partial f}{\partial p}+D_{p \theta} \frac{\partial f}{p \partial \theta}\right)+\frac{\partial}{p \sin \theta \partial \theta} \sin \theta\left(D_{\theta p} \frac{\partial f}{\partial p}+D_{\theta \theta} \frac{1}{p} \frac{\partial f}{\partial \theta}\right)\right] \\
D_{p p} & =\int d^{3} \mathbf{k} \sum_{l} W_{\mathbf{k}}(\mathbf{r}, t) U_{l}(\mathbf{p} ; \mathbf{k}) \delta\left(\omega-k_{\|} v \cos \theta-l \omega_{c} / \gamma\right) \\
D_{\theta \theta} & =\int d^{3} \mathbf{k} \sum_{l}\left(\frac{l \omega_{c} / \gamma-\omega \sin ^{2} \theta}{\omega \sin \theta \cos \theta}\right)^{2} W_{\mathbf{k}}(\mathbf{r}, t) U_{l}(\mathbf{p} ; \mathbf{k}) \delta\left(\omega-k_{\|} v \cos \theta-l \omega_{c} / \gamma\right) \\
D_{\theta p}= & D_{p \theta}=\int d^{3} \mathbf{k} \sum_{l}\left(\frac{l \omega_{c} / \gamma-\omega \sin ^{2} \theta}{\omega \sin \theta \cos \theta}\right) W_{\mathbf{k}}(\mathbf{r}, t) U_{l}(\mathbf{p} ; \mathbf{k}) \delta\left(\omega-k_{\|} v \cos \theta-l \omega_{c} / \gamma\right) \\
& \frac{d W_{\mathbf{k}}(\mathbf{r}, t)}{d t}=2 \Gamma_{b} W_{\mathbf{k}}(\mathbf{r}, t) \\
& \Gamma_{b}=\frac{1}{2} \int d^{3} \mathbf{p} \sum_{l} U_{l}(\mathbf{p} ; \mathbf{k}) \delta\left(\omega-k_{\|} v \cos \theta-\frac{l \omega_{c}}{\gamma}\right) v\left[\frac{\partial f}{\partial p}+\frac{\frac{l \omega_{c}}{\gamma}-\omega \sin ^{2} \theta}{\omega \sin \theta \cos \theta} \frac{\partial f}{p \partial \theta}\right]
\end{aligned}
$$

where $f$ is the particle distribution function in phase space, and $W_{\mathbf{k}}(\mathbf{r}, t)$ is the local spectral energy density of the waves. For waves in cold plasma, the $U_{l}(\mathbf{p} ; \mathbf{k})$ factor is

$$
U_{l}(\mathbf{p} ; \mathbf{k})=8 \pi^{2} e^{2} \frac{\left\{\frac{l \omega_{c}}{k_{\perp} v \gamma} J_{l}+E_{3} \cos \theta J_{l}+i E_{2} \sin \theta J^{\prime}{ }_{l}\right\}^{2}}{\left(1-E_{2}^{2}\right) \frac{1}{\omega} \frac{\partial}{\partial \omega} \omega^{2} \varepsilon+2 i E_{2} \frac{1}{\omega} \frac{\partial}{\partial \omega} \omega^{2} g+E_{3}^{2} \frac{1}{\omega} \frac{\partial}{\partial \omega} \omega^{2} \eta}
$$

where the argument of the Bessel function $J_{l}$ and its derivative ${J^{\prime}}_{l}$ is $k_{\perp} v \gamma \sin \theta / \omega_{c}$.

The total time derivatives in Eqs. (131), 132 are derivatives along the unperturbed particle orbit and the wave-packet trajectory, respectively. The latter is governed by the WKB equations (120, 121), so that

$$
\frac{d W_{\mathbf{k}}(\mathbf{r}, t)}{d t} \equiv \frac{\partial W_{\mathbf{k}}(\mathbf{r}, t)}{\partial t}+\frac{\partial \omega}{\partial \mathbf{k}} \frac{\partial W_{\mathbf{k}}(\mathbf{r}, t)}{\partial \mathbf{r}}-\frac{\partial \omega}{\partial \mathbf{r}} \frac{\partial W_{\mathbf{k}}(\mathbf{r}, t)}{\partial \mathbf{k}}
$$

Equations 131), 132 conserve the number of particles, $\int f d^{3} \mathbf{p} d^{3} \mathbf{r}$ and the total energy of particles and waves, $\int f \sqrt{p^{2} c^{2}+m^{2} c^{4}} d^{3} \mathbf{p} d^{3} \mathbf{r}+\int W d^{3} \mathbf{k} d^{3} \mathbf{r}$. These conservation laws hold irrespective of the emission probability specified by $U_{l}(\mathbf{p} ; \mathbf{k})$.

Once developed, a numerical code for solving Eqs. (131) and 132 will enable accurate predictions of micro-instability impact on the spatially global evolution of the runaway electrons in a tokamak, where excitation of whistler waves via anomalous Doppler resonance is 
the primary candidate for enhanced scattering of the runaways. Such a code would naturally complement the gyrokinetic codes that are not designed to model high-frequency kinetic instabilities. It will also have broader applications to problems in which fast instabilities govern electron transport. 


\section{EXPERIMENT}

Experimentally, runaway electrons have been observed and studied from the very beginning of experiments in toroidal devices [7]. There exists therefore a vast body experimental work related to REs in tokamaks spanning multiple decades, and any review must necessarily be limited in scope. Overall, REs have been observed in the energy range from the thermal plasma temperature (keV range) to highly relativistic energies (several 10s of MeV). Generally, the energy distribution appears to fall with increasing energy, although evidence for non-monotonic (beam-like) energy distributions has been observed. The toroidal current carried by the REs varies from very small (several $\mathrm{kA}$ for QREs or disruption RE seeds) to very large (nearly $1 \mathrm{MA}$ for largest post-disruption RE plateaus). Here, an overview of RE diagnostics is given in Section XA. An overview of quiescent runaway electron experiments is given in Section XB. Post-disruption RE plateaus and their dissipation are introduced in Section XC, while RE plateau position control and vertical stability are discussed in Section XD, RE seed formation during disruptions is discussed in Section XE, while the

prompt loss of these RE seeds due to magnetic perturbations is discussed in Section XF. Experimental work on the RE avalanche and its suppression is discussed in Section X G. Finally, interactions between REs and the first wall are discussed in Section XH.

\section{A. RE diagnostics}

Runaway electrons (REs) span a broad energy range from the thermal electron energies (of several keV during a typical discharge) to tens of $\mathrm{MeV}$, thought to be limited by synchrotron emission, ripple loss, and drift orbit loss [167]. This wide energy range cannot be surveyed by any single diagnostic. A variety of diagnostics are therefore used to characterize the RE energy, pitch angle, and spatial distributions [7, 168 172].

\section{Bremsstrahlung}

Bremstrahlung emission is one of the most widely used diagnostic tools for diagnosing REs in tokamaks [75, 173 176]. Strictly speaking, "bremsstrahlung" refers to any radiation due to slowing of charged particles, but we will use bremsstrahlung here in the more narrow sense of radiation from electrons slowing in matter. Bremstrahlung from REs can be "thin 
target" volumetric bremsstrahlung when REs are scattered by plasma ions or "thick target" bremsstrahlung when REs hit plasma-facing components. Volumetric emission dominates when REs are well confined, whereas surface bremsstrahlung prevails during times of rapid RE loss such as MHD-related prompt loss or RE plateau final loss [174], or if the diagnostic is viewing a limiter [170, 177]. The energy spectrum of emitted photons has an upper boundary at the incident electron energy and a peak at about $1 / 4$ of the incident electron energy [178]. The angular distribution of bremsstrahlung photons tends to be isotropic under $1 \mathrm{MeV}$ (soft and mid x-ray ranges, SXR and MXR) and become forward-directed above $1 \mathrm{MeV}$ (hard $\mathrm{x}$-ray, HXR range) [179]. Electron-ion collisions dominate over electron-electron collisions in producing bremsstrahlung, and the bremsstrahlung thus has a strong $Z^{2}$ nuclear charge dependence, which makes the emission sensitive to the impurity content of the plasma [178.

Detection of bremsstrahlung photons is typically done using semiconductor detectors at lower energies and scintillation detectors at higher energies. In the SXR range (1 keV to 10 $\mathrm{keV}$ ), Si detectors are most common in tokamaks [180], while in the MXR range (10 keV to $1 \mathrm{MeV}$ ), CdTe detectors are more commonly used due to their higher stopping power [170. Semiconductor detectors directly detect current from electron-hole pairs created by the photons and can achieve very high photon detection efficiencies (up to unity quantum efficiency in some cases). Scintillation detectors use high-density, transparent materials to detect visible light emission resulting from HXR impact. The visible light emission is due to recombination of electrons into bound states, while the HXR energy absorption mechanisms are the photo-electric effect (for photons under $1 \mathrm{MeV}$ ), Compton scattering at intermediate energies, and electron-positron pair production at highly relativistic energies (> $10 \mathrm{MeV}$ ) [181]. Scintillator materials used in tokamaks include NaI(Tl) [182 184], BGO [75, 174], and $\mathrm{LaBr} 3(\mathrm{Ce})$ [185]; different scintillators are chosen based on criteria such as signal level, signal decay rate, and neutron vs. HXR discrimination.

Counting of signals from bremsstrahlung photons can be done in either "current mode" or "spectroscopy mode". In current mode, the total photo-current (usually proportional to photon energy flux) is simply measured [174]. In spectroscopy mode, individual photon pulse heights are measured, allowing statistical reconstruction of the photon energy distribution function [186]. Spectroscopy mode therefore provides more information, but is more challenging experimentally, requiring higher gains, better shielding against noise, and better collimation. 
Shielding (blocking scattered HXR signals) and collimation (narrowing field of view to a specific view chord) are a challenge, in general, when measuring RE bremsstrahlung emission, as the HXR portion of the photon spectrum is extremely hard to block, requiring several $\mathrm{cm}$ of lead shielding to attenuate significantly. Often, HXR spectra are taken far from the machine with a perpendicular viewing angle [176]. This reduces shielding requirements but also makes interpretation of the received signal more challenging, as only a small fraction of the HXR photons are emitted into the perpendicular direction. An attempt has been made to perform RE HXR spectroscopy with a tangential viewing geometry in DIII-D; this geometry is superior for capturing the high-energy portion of the HXR emission, but HXR

fluxes are higher and shielding and collimation requirements are quite challenging, requiring thick $\sim 10 \mathrm{~cm}$ lead shielding and also thick $\sim 10 \mathrm{~cm}$ long tungsten view chord collimation tubes [75].

In JET, where multiple vertical HXR views are available from two poloidal locations, tomographic inversions have been performed to recover the spatial distribution of HXR emissivity in the plasma volume [187.

\section{Synchrotron emission}

In the tokamak community, "synchrotron emission" typically refers to light emission by relativistic electrons in a magnetic field, while "cyclotron emission" refers to light emission by non-relativistic electrons in a magnetic field. This nomenclature is not completely consistent with other fields; for example, in the astrophysics community, "synchrotron radiation" often refers to radiation due to electron gyro-motion around a magnetic field line, while "curvature radiation" refers to electron motion along a curved magnetic field line, and "synchro-curvature radiation" refers to the general case of helical motion along a curved field line [188]. In medium to large-sized tokamaks, the electron gyro-motion is typically the prevailing factor. In this regime, synchrotron emission power depends strongly on RE kinetic energy $\left(\right.$ as $\left.\gamma^{4}\right)$ and pitch angle $\left(\right.$ as $\left.\theta^{2}\right)$ [61, 65]. The pitch angle itself depends on the plasma impurity content, resulting in synchrotron emission, which tends to increase strongly with the amount of impurities. For typical observed near-equilibrium post-disruption RE energy distribution functions in mid-sized tokamaks, synchrotron emission comes predominantly from REs in the $30-40 \mathrm{MeV}$ energy range and peaks in the mid-IR $(2-4 \mu \mathrm{m})$ 
wavelength range [189]. Emission is very strongly forward-beamed, requiring a tangential viewing system. Tangential synchrotron emission from tokamak REs was first observed in the TEXTOR tokamak using an IR camera [169] and has since been used in e.g. C-MOD [190] and DIII-D [191, 192] using imaging with the visible and IR cameras. To-date, synchrotron emission spectra have been measured in the visible [191], but not in the IR. The use of synchrotron emission polarization as a diagnostic tool has been proposed [66] and first measurements of this were made at C-MOD [190].

\section{Cyclotron emission}

Measurement of electron cyclotron emission (ECE) in the microwave $(\sim 50-200 \mathrm{GHz})$ frequency range is one of the earliest tokamak diagnostics [168]. It employs either fast systems (radiometers) or slow systems (interferometers). Fast ECE diagnostics typically use heterodyne radiometers to split the microwave signal into separate channels, each with a separate bandpass filter to isolate a specific frequency band [193], giving very fast $(>1$ $\mathrm{MHz})$ measurements of ECE emission with coarse $(\sim 1 \mathrm{GHz})$ spectral resolution. Slower $(<100 \mathrm{~Hz})$, broadband, and higher spectral resolution $(<1 \mathrm{GHz})$ ECE measurements are usually obtained with Michelson interferometers [168, 194]. ECE measurements are usually made at a single polarization, either perpendicular to the toroidal magnetic field or parallel to the magnetic field. The observation direction is typically radially inward, in order to measure the plasma radial temperature profile.

Fast electrons increase ECE signal levels and cause a broadening of the distribution function, making ECE a potentially useful diagnostic for RE analysis [195]. To-date, however, the complexity of interpreting RE ECE contributions has limited RE ECE to mostly qualitative use. The rise of ECE signals relative to Thomson scattering was used to confirm the growth of trace REs in low density quiescent runaway electron (QRE) discharges [196]. During RE-producing disruptions, clear strong spikes in ECE emission associated with RE seeds are frequently observed [174]. During RE plateaus in DIII-D, ECE spectra indicated that lower energy REs had a higher mean pitch angle than higher energy REs [172].

Some diagnostic improvements have been proposed and implemented to improve usefulness of ECE for fast electron measurements. High-field side ECE has been implemented in TCV [171]. This gives improved protection against cutoff of fast electron ECE emission: 
because of the relativistic downshift in gyro-frequency, fast electrons are more susceptible to density cutoff than thermal electrons. Measuring ECE from the high field side, with higher gyro-frequency, can in some cases allow measurement of fast electron emission which would be absorbed before reaching the low field side. Vertical ECE measurement was demonstrated in C-MOD [197] and proposed for TCV [198]: measuring ECE along a vertical chord loses profile information, but gains information on the fast electron energy distribution, since only a single major radius (and single non-relativistic gyro-frequency) is imaged so broadening of the ECE signal is dominantly due to the energy spread along the view chord. A major challenge of this approach is avoiding reflections from the metal wall, so a viewing dump is normally used. "Oblique" ECE, i.e. a tangential or partially tangential viewing angle, has also been proposed for increased sensitivity to non-thermal electrons, and was implemented in the PBX-M tokamak [199]. While thermal ECE is emitted with a very broad angular distribution, non-thermal ECE becomes increasingly forward-beamed, so viewing tangentially gives stronger signal from non-thermal electrons. As with vertical ECE, removal of wall reflections is important to make oblique ECE data interpretation tractable.

\section{Line emission}

In principle, impurity line emission can be used to diagnose REs, but little work has been done in this area. In the RE plateau of DIII-D, it was concluded that visible line emission was dominantly due to excitation by or recombination with cold thermal electrons, limiting the usefulness of visible lines as RE diagnostics in this regime [172]. A more promising approach could be the use of more energetic line emission that requires fast electrons to excite. For example, in early fast electron studies, $12.6 \mathrm{keV}$ Krypton K $\alpha$ emission was used to diagnose fast electrons in a discharge after puffing krypton gas [200].

\section{Probe diagnostics}

Potential damage to plunging probes complicates the use of direct probes as RE diagnostics; nevertheless, probes have been used successfully in the limiter shadow (scrape-off layer) of TEXTOR plasmas to diagnose boundary REs.

A scintillator probe design was developed in TEXTOR, which used a reciprocating probe 
to insert the probe tip into the edge plasma (scrape-off layer) of RE-containing shots. The probe tip contained ten YSO (Y2SiO3:Ce) crystals coupled with optical fibers to photomultiplier tubes. The scintillators were shielded with differing amounts of tungsten shielding. Simulation of the scintillator signals was used to reconstruct the energy spectrum of boundary REs striking the probe. The simulations indicated that HXR activation of the scintillators was negligible (compared with RE impact), so the probe signals provided a fairly direct measurement of RE impact. The energy range of this diagnostic spanned approximately 5 $-20 \mathrm{MeV}$ [201].

Cherenkov probes are somewhat similar to scintillator probes in design in the sense that a probe tip containing a shielded transparent material is coupled with optical fibers to photodetectors. Material choices are different though, to optimize Cherenkov emission rather than scintillation; for example, AlN and diamond are typical Cherenkov detector materials. Cherenkov radiation is produced in any material when struck by particles with superluminal velocity, e.g. electron energies $>58 \mathrm{keV}$ in diamond or $>175 \mathrm{keV}$ in plastic. Often, both scintillations and Cherenkov emission are present and the two signals sometimes need to be separated out from each other to avoid confusion [202]. Relative to scintillator probes, Cherenkov probes tend to have the advantage of faster time response $(<1 \mathrm{~ns}$, compared with $>100 \mathrm{~ns}$ for typical scintillators) but lower signal levels [202]. Cherenkov probes have been implemented in CASTOR, ISTTOK, Tore Supra, COMPASS, and FTU tokamaks [203, 204].

A different probe technique, also used in the TEXTOR edge plasma, was a calorimeter probe, which used the heating profile of REs striking the probe to infer RE heat flux. The probe consisted of a layered stack starting with carbon fiber composite, then graphite, and then molybdenum (to absorb remaining highest energy REs), with thermocouples connected through the stack. Heat fluxes were found to be consistent with estimates of the scintillator probe [205].

\section{IR thermography}

Infra-red imaging has the advantage over probes that the measurement is remote and can be used to study the structure of the RE heat loads over extended regions of the wall; this has been done in JET [206] and DIII-D [207]. In principle, analysis of IR imaging could deduce the energy flux carried by REs when they strike plasma-facing components; 
in practice, quantitative analysis of the IR images is quite challenging. In addition to the well-known complications of IR thermography with thermal electron heat loads (non-ideal first wall material properties including surface morphology, layers and impurities [208, 209], plasma IR line emission [210], and reflections [211]), IR thermography of RE heat loads must deal with the nontrivial dependence of the RE penetration depth into the material on pitch angle and energy, because deeper RE energy deposition can be obtained either by turning up energy or increasing pitch angle [207].

\section{Neutron emission}

Under normal operation, tokamak neutron emission is typically dominated by D-D fusion reactions, especially in the presence of beam heating. However, in the presence of sufficiently large RE populations or when using $\mathrm{H}$ or He plasmas, neutron production can be primarily due to $(\gamma, n)$ photo-neutron nuclear reactions in the wall material struck by REs. The photoneutron production is usually dominated by "giant" resonances at high energies s (e.g. at $22.5 \mathrm{MeV}$ for ${ }^{12} \mathrm{C}[212]$ ). Typical detectors are $\mathrm{BF}_{3}$ counters or U-235 fission chambers which tend to have slow $(<1 \mathrm{~ms})$ time responses [170]. Scintillators can also be used to detect photo-neutrons; these can have fairly high time response $(<1 \mu \mathrm{s})$ but have the disadvantage of measuring both neutrons and HXRs, so baseline subtraction is usually needed to separate photo-neutron from HXR signals [213]. Photo-neutron Cherenkov radiation has also been used to develop a measurement of RE-wall strikes with fast (several $\mu \mathrm{s}$ ) time response in KSTAR [214]. Photo-neutron signal is typically used as evidence of the loss of highenergy REs from the plasma, without any energy resolution, although a more detailed study including the measurement of the photo-neutron spectrum with a ${ }^{3} \mathrm{He}$ counter was done in the PLT tokamak. The measured spectrum was found to be continuous with a peak energy $<2 \mathrm{MeV}$ and was seen to rise when RE energy passed the expected threshold energy $\sim 10$ $\mathrm{MeV}$ for photo-neutron production reactions in the $\mathrm{W}$ limiter [215].

\section{Laser scattering}

Thomson scattering of laser light off electrons is a well-known tokamak diagnostic and the ability of Thomson scattering to see non-Maxwellian (but non-relativistic) electrons was 
pointed out at the C-MOD tokamak [216]. The relativistic analog, laser-induced inverse Compton scattering (LICS), has been proposed for RE diagnosis [217] but has not been implemented yet. In principle, LICS could give radial profiles of RE density, much as Thomson scattering does presently for thermal electrons. This measurement will be very challenging, however, since the scattered light is upshifted into the SXR energy range where there is a large bremsstrahlung background. Successful implementation of LICS will therefore require powerful ultra-short pulse lasers and sensitive, synchronously gated SXR detectors.

\section{9. $\quad$ Pellet injection}

Pellet injection is a possible method for diagnosing REs, although the very rapid destruction of pellets by REs somewhat limits the usefulness of this technique. One use of pellets is to identify the edge of the RE beam, i.e. the location at which a small injected pellet is destroyed; this was done in some DIII-D experiments using small polystyrene pellets [174]. In different DIII-D experiments, the same Ar pellet used to disrupt the plasma and form the RE beam was also used as a diagnostic tool, i.e. the pellet survived the TQ and RE formation phase and then continued through the plasma until being destroyed by RE seeds on the high field side [207].

\section{B. Startup and quiescent runaway electrons}

REs can be created at discharge startup in tokamaks if insufficient pre-fill gas or very large RF current drive is present 218. Generally, tokamak experiments try to avoid startup REs or suppress them during the shot by raising density, but in some discharges they nevertheless survive. A full range of relative RE population levels have been observed in startup RE shots, ranging from nearly thermal (trace REs), to "slide-away" discharges (with reduced resistivity, but thermal plasma still at high temperature and still carrying current), to full RE beams (with toroidal current dominated by REs, and background thermal plasma cold). Tokamak discharges with startup REs have been studied extensively for decades. Early findings of these studies have been reviewed by [7].

Kinetic instabilities are observed frequently in startup REs, often manifesting as periodic relaxation oscillations, with rises in loop voltage correlated to HXR spikes [151, 219]. 
These relaxation oscillations have been interpreted as a nonlinear interplay of two kinetic resonances: the Cherenkov resonance (which scatters REs down in energy when $\frac{\partial f}{\partial v_{Z}}>0$ ), and the anomalous Doppler resonance (which scatters REs in pitch angle when $T_{\|}>T_{\perp}$ ) [8].

Transport loss of startup REs was studied in TM-3, LT-3, ORMAK, and Tuman-2 tokamaks. In some cases, transport was consistent with a simple drift-orbit loss model, particularly for higher-energy (multi-MeV) REs [220], but in other cases it was necessary to supplement the drift-orbit loss with a radial diffusion coefficients as high as $D_{\perp} \approx 1-10$ $\mathrm{m}^{2} / \mathrm{s}$ to match data [221, 222]. In the HT-7 tokamak, startup REs in the boundary region were observed to be lower energy $(<5 \mathrm{MeV})$ compared with the core; this was interpreted as being due to rapid radial loss of high energy REs in the edge field ripple [182]. In the COMPASS tokamak, startup REs have been shown to have enhanced loss in the presence of MHD activity, such as sawteeth [223]. A review of fast electron loss (theory and experiment) in the presence of magnetic turbulence was presented previously [224].

In the last several years, fundamental RE measurements in tokamaks have focused less on startup REs and more on quiescent runaway (QRE) discharges. QRE discharges are dedicated RE-forming discharges, created by slowly ramping down electron density until REs appear. These shots therefore enter the trace RE regime in a controlled fashion. In the trace RE regime, thermal plasma still dominates in terms of number density and current density, so that standard tokamak diagnostics (charge exchange recombination, Thomson scattering, etc) enable excellent characterization of the background plasma. QRE discharges therefore serve as good test beds for the study of fundamental RE physics, such as synchrotron emission, collisional damping, and kinetic instabilities.

One significant finding of the QRE studies has been that the driving electric field needed for runaway production appears to be unexpectedly high. This was observed by looking for RE onset at varying background densities in DIII-D, C-MOD, FTU, KSTAR, and TEXTOR [225]. Reference [225] provides a complete list of parameters chosen for these comparative studies. Figure 33(a) presents an overview of the resulting data. In these experiments, the density is lowered slowly until REs are first observed. The parallel electric field at this point was expected to be close to the Connor-Hastie value $E_{C H}$ determined by electron-electron collisional drag [40]. However, as can be seen in Fig. 33, the observed threshold field is 5-10 times larger than $E_{C H}$. 
The exact source of this discrepancy is not certain yet. In C-MOD and FTU, comparison with modeling has indicated that synchrotron damping is sufficient to explain the anomalously high measured damping [226 228].

Another possible source of the discrepancy is the time-dependence of the experiments. The measurements are necessarily time-dependent and additionally the detection threshold of the runaway electrons is substantial for any diagnostic (e.g., synchrotron emission or HXR emission). As the density is decreased, trace runaway electrons should emerge at some point; however, they might not actually increase to a level that can be observed until the density has gone down further, resulting in misinterpretation of the data. As discussed in Section $\mathrm{VA}$, the electric field has to exceed $1-2 \%$ of the Dreicer field $E_{D}$ to produce an adequate number of seed runaway electrons to be observed at all (regardless of diagnostic signal/noise) on the QRE experimental time scales of $\sim 1$ second. The electron temperature ranged from 0.75 to $2 \mathrm{keV}$ in the DIII-D QRE experiments resulting in the $E / E_{D}$ ratio of $0.5 \%$ to $2 \%$, so the experiments take place in a regime which RE growth time scales are marginal.

To avoid this growth time scale issue, a growth/decay method was developed [229]. This method uses the observed growth rate of the runaway electron population when the avalanche is expected to dominate. Fitting the observed growth rate as a function of the electric field gives a curve that intersects zero at $E \sim 4 E_{C H}$. Comparing with the onset method, this is a factor of 3 lower for DIII-D in Fig. 33(a). It is therefore conceivable that the discrepancy between the measured electric field and $E_{C H}$ is actually $2-3$ times rather than the 5-10 obtained from the onset experiments. Another interesting observation in DIII-D is that the measured growth rate depends on the energy range being measured. The growth rate was measured at different energies in DIII-D and was found to be closer to theory at higher energies, which is a sign of enhanced RE dissipation at low energies in QRE discharges [76]. This suggests that, at least in these experiments, neither synchrotron damping, nor drift orbit losses are responsible for anomalous RE dissipation in, but rather a factor acting on lower energy REs. Possible candidates could be errors in the collisional drag and/or avalanche gain evaluation, kinetic instabilities which increase pitch angle (thus reducing current), or a radial transport mechanism which is larger for lower energy REs (say, fluctuations with a length scale comparable to the drift orbits of lower energy REs). The presence of kinetic instabilities in QRE experiments was confirmed with high frequency (100 - 
(a)
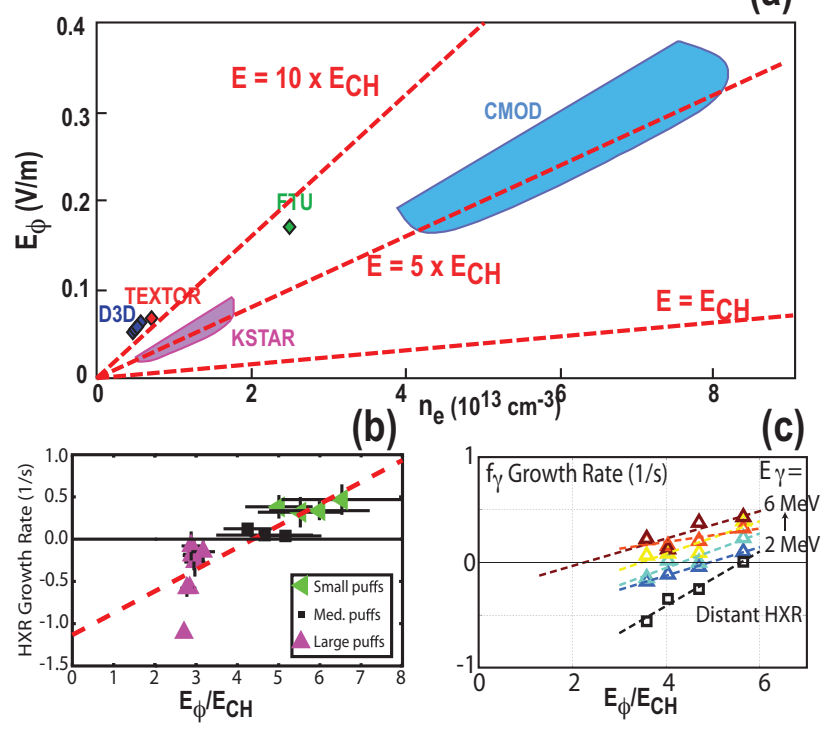

FIG. 33. (a) Overview of threshold toroidal electric field needed to observe RE onset in QRE experiments on different machines, as a function of electron density [225], (b) Improved estimate of threshold toroidal electric field for RE formation in DIII-D using growth/decay method, and (c) growth rate of HXR photon signals in DIII-D showing different growth rates at different energies [76].

$200 \mathrm{MHz}$ ) magnetics measurements in DIII-D. The density and magnetic field scaling of the observed magnetic oscillations were consistent with the whistler wave dispersion relation [154. Recent quasi-linear simulations [161 allegedly support the idea that whistlers are responsible for the high avalanche threshold field observed in DIII-D, but more work by the community is needed to ensure quantitative applicability of the numerical model and interpret the data unambiguously.

\section{Plateau runaway electrons and their dissipation}

We define plateau REs as REs which are formed after a tokamak disruption at sufficient level to dominate the plasma current. The RE plateau is thus somewhat similar to startup RE beams which can form with very low density startup. The post-disruption RE plateau is thought to establish via avalanche amplification from RE seeds formed during thermal quench (TQ), as discussed in the next section. RE plateaus form more readily in some 
tokamaks and some experimental conditions than others. The observed trends are largely (albeit not completely) understood, at least qualitatively. A clear trend seen in all machines is that disruptions with higher-Z impurities are more likely to form a RE plateau [230]. This is because higher- $\mathrm{Z}$ impurities cause a faster temperature collapse and higher loop voltage, both expected to contribute to greater RE seed formation and amplification. This trend applies to intentionally injected impurities, as well as to those coming from the wall. For example, the change from a carbon to a beryllium first wall in JET resulted in a lower likelihood of RE plateau formation following unmitigated disruptions [231]. Another trend seen across machines is that large machines with almost circular flux surfaces are more likely to form a RE plateau. The smaller and elongated discharges are more prone to magnetic island overlap and flux surface stochastization following the TQ, resulting in greater "prompt loss" of seed REs to the wall [232].

The plateau REs tend to form a narrow circular beam; this has been seen from SXR arrays [172, 233], from synchrotron imaging [191, 210, and from intentional release of the $\mathrm{RE}$ plateau into the divertor [172]. The current profile of the RE plateau has not been measured directly, but is frequently assumed to follow the SXR profile as a rough guess [136].

Low-resolution, wide-range $(5 \mathrm{keV}-50 \mathrm{MeV})$ estimates of the energy distribution function $f(\mathcal{E})$ of the RE plateau have been made in DIII-D by piecing together data from SXR, MXR, HXR, IR synchrotron, and visible synchrotron diagnostics, as well as including constraints from measured plasma current and total radiated power, Fig. 34(a). The approximate sensitivity ranges of the different diagnostics used is shown in Fig. 34(b). It can be seen that high energies are strongly constrained by synchrotron emission, while low energies are constrained by SXR and radiated power, but medium energies $(\sim 1 \mathrm{MeV})$ are poorly constrained. It was found [210] that $f(\mathcal{E})$ differed significantly from what one would have transiently in a rapidly growing avalanche. The latter is shown by the dot-dash curve in Fig. 34 (a) and has a form $f \sim \exp \left(-\frac{\sqrt{\mathcal{E}\left(\mathcal{E}+2 m c^{2}\right)}}{46 m c^{2}}\right)$ when drag on REs is dominated by bound Ar electrons [5]. Subsequent test particle modeling of the DIII-D experimental conditions provided a better match to the data, Fig. 34(d). For this comparison, the measured distribution at $t=1490 \mathrm{~ms}$ was used as initial condition and the measured electric field as an input. The distribution at $t=1730 \mathrm{~ms}$ was then calculated numerically from the relativistic kinetic equation with secondary electron production, synchrotron drag, and drift-orbit losses 
considered [207]. It can be seen that the energy distribution function is close to steady-state, i.e. the transient avalanche distribution is not even expected to be representative here. The dip in the measured distribution function, Fig. 34(a), around $1 \mathrm{MeV}$, is in a region which is poorly constrained, with large error bars, so improved diagnostics in the $1 \mathrm{MeV}$ range are needed for better reconstruction of $f(\mathcal{E})$ here.

The green dot-dash curve in Fig. 34(c) is the predicted mean pitch angle assuming steadystate force balance between the toroidal electric field $E_{\phi}$, which tends to reduce pitch angle, and collisions, which tend to increase pitch angle.

The predicted pitch angle distribution is then given by Eq. (106) (see Section VII and Ref. [210] for details), and the averaged value of $\cos \theta$ is $\langle\cos \theta\rangle=\operatorname{coth} A-1 / A$, where $A(p)$ is defined by Eq. (107).

In JET, a more narrow range $(1 \mathrm{MeV}-20 \mathrm{MeV})$, higher resolution estimate of plateau $f(\mathcal{E})$ was made using HXR pulse-height counting with a radially inward view. Peak RE energies of order $15 \mathrm{MeV}$ were observed. Two distinct energy components were inferred in some cases (although never with a no-particle gap between the two). The two components were conjectured as being the primary (seed) and secondary (avalanche-produced) RE's [176].

Estimates of the total energy of the RE plateau typically give values ranging from $30 \mathrm{~kJ}$ in TEXTOR to several $100 \mathrm{~kJ}$ in JET [234], with a linear increase in RE beam kinetic energy with RE current [205]. This linear dependence suggests that the shape of the runaway distribution function is the same for all values of the runaway currents. A quadratic increase in RE plateau magnetic energy with RE current is expected in this case. The RE plateau magnetic energy is typically larger than the RE kinetic energy in present experiments, and the RE plateau current is correspondingly large compared with the Alfven current (17 kA).

Pitch angle estimates of $\mathrm{RE}$ plateaus have been made, arriving at $\theta \approx 0.1-0.2$ for high energy (multi-MeV) REs based on the elongation of synchrotron images in TEXTOR and DIII-D [191]. Interpretation of microwave ECE spectra suggested a large mean pitch angle $(\theta \sim 1)$ for low energy ( $\sim 100 \mathrm{keV}$ range) REs in DIII-D [172]. The multi-diagnostic (SXR, MXR, HXR, synchrotron) analysis done in DIII-D also indicated a large pitch angle at low energy dropping to $\theta \approx 0.1$ at high energy, as shown in Fig. 34(c), consistent with the notion that pitch angle shrinking due to electron acceleration in the inductive electric field is balanced by the collisional scattering. 


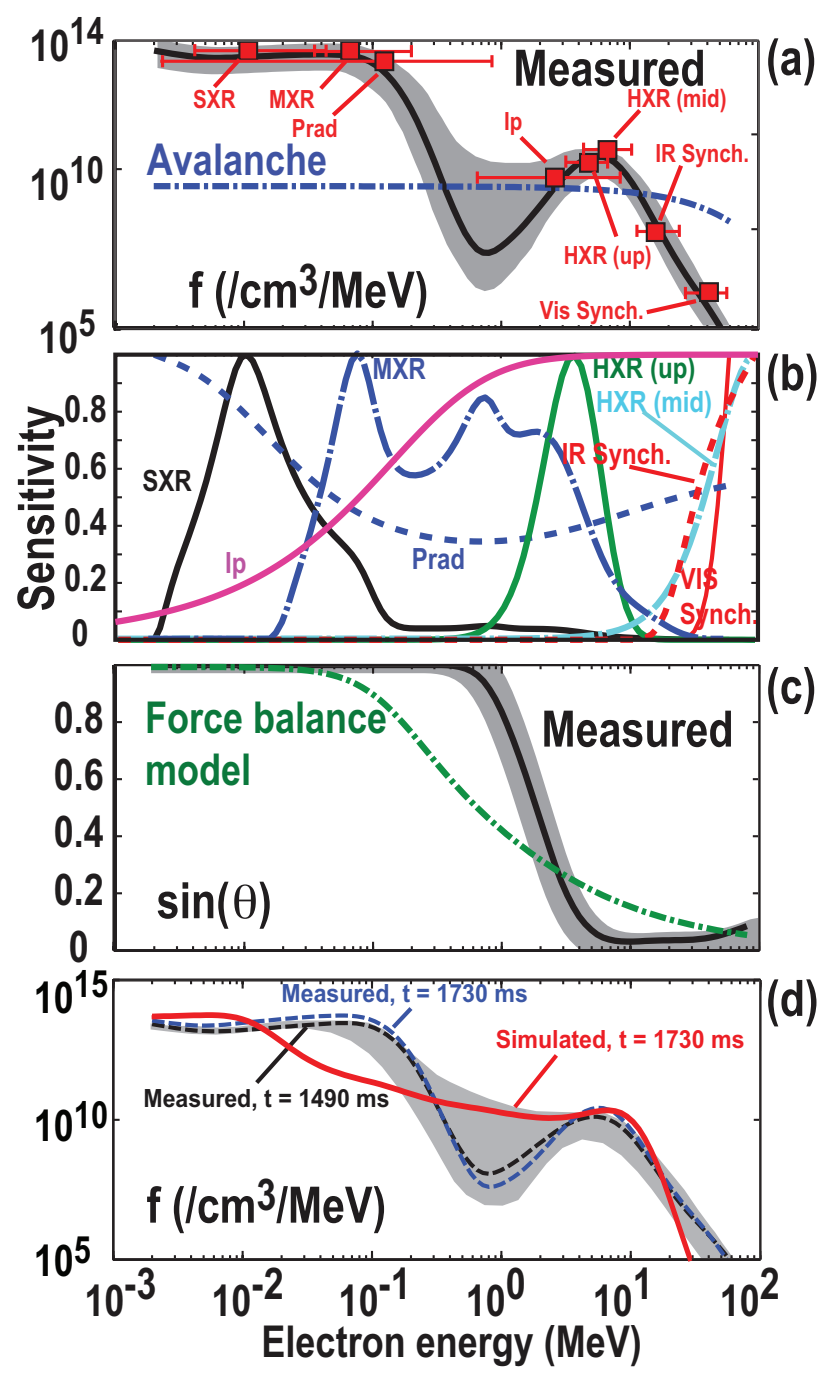

FIG. 34. (a) Runaway electron energy distribution function reconstructed from multiple diagnostics during DIII-D RE plateau; (b) approximate relative sensitivities of different diagnostics; (c) mean pitch angle reconstructed for the same data; and (d) the measured energy distribution compared with a numerically simulated energy distribution. Plots (a,b,c) from [210] and plot (d) from [207].

The current damping of the RE plateau is of fundamental importance, since it affects RE plateau energy dissipation and vertical displacement timescale.

Similar to QRE experiments, the damping of RE current in the RE plateau appears to be larger than expected from inelastic collision drag on free + bound electrons. This can be measured by applying sufficient external loop voltage to hold the RE plateau current constant. In a steady state with $\dot{I}_{P}=0$, the external and internal loop voltage are expected 
to be equal, so the toroidal electric field in the plasma is known from external sensors. Figure 35 (a) shows the measured steady-state electric field as a function of impurity content represented by mean ion charge $\langle Z\rangle$. At highest impurity levels, $\langle Z\rangle>10$, it is no longer becomes possible to balance the high background drag with the applied electric field, and the $\mathrm{RE}$ current decays. Interpretation of this data is challenging because $\dot{I}_{P} \neq 0$, so the internal electric field is not known. Because of the large plasma inductance, it is expected that the decaying plasma current will produce a counter-emf which will increase internal $E_{\phi}$. As a rough approximation, $E_{\phi} \approx E_{\phi, w a l l}-\frac{\dot{I}_{p}}{I_{p}} \frac{\bar{p}}{e}$, where $\bar{p}$ is the characteristic RE momentum. The resulting points are shown as empty inverted triangles in Fig. 35(a), labeled "corrected" data.

Overall, it can be seen that $E_{\phi}$ is typically $2-10 \times E_{C H}$ indicating that the steady-state drag on the REs is of order $2-10 \times$ larger than the purely electron drag (with bound electrons counted as $1 / 2$ free electrons). This discrepancy was previously interpreted as an indication that pitch angle scattering off high-Z nuclei could be important [210]. Section $\amalg I I C$ of this review presents theoretical models of screened impurity nucleus pitch angle scattering. The corresponding threshold electric field $E_{0}$, given by Eq. (105) in combination with Eqs. (101) and (103), is shown by red markers in Fig. 35(a). In Eq. 105) the $\nu_{e l}$ is evaluated using Eqs. (40), and Coulomb logarithms in $\nu_{d r a g}$ are evaluated according to Eqs. 27) and (29). Although pitch angle scattering raises the threshold field above $E_{C H}$, it is still not sufficient to match the data at medium $Z$, suggesting consideration of radial transport losses.

In addition to pitch angle scattering, it is possible that the energy dependence of inelastic scattering could explain the discrepancy of Fig. 35(a). The avalanche concept implies relativistic $(\mathrm{MeV}$ level), growing runaway electron current, while the experiments exhibit a broad, continuously re-filled distribution function going down to keV level energies. If significant current is carried by keV energy electrons, then the stopping power can be enhanced accordingly.

Also, the role of radial transport has not been well-quantified in the experiments. Radial transport loss is clearly present in the RE plateau, but it does not seem to be the dominant factor for centered RE plateaus where line radiation balances Joule heating. Yet, this balance ceases when the RE plateau shifts inward in major radius toward the wall, giving a rise in plasma resistivity (current damping rate) [172]. The power balance data indicate that dissipation of the RE plateau is predominantly internal (not to the wall) for centered, mid- 


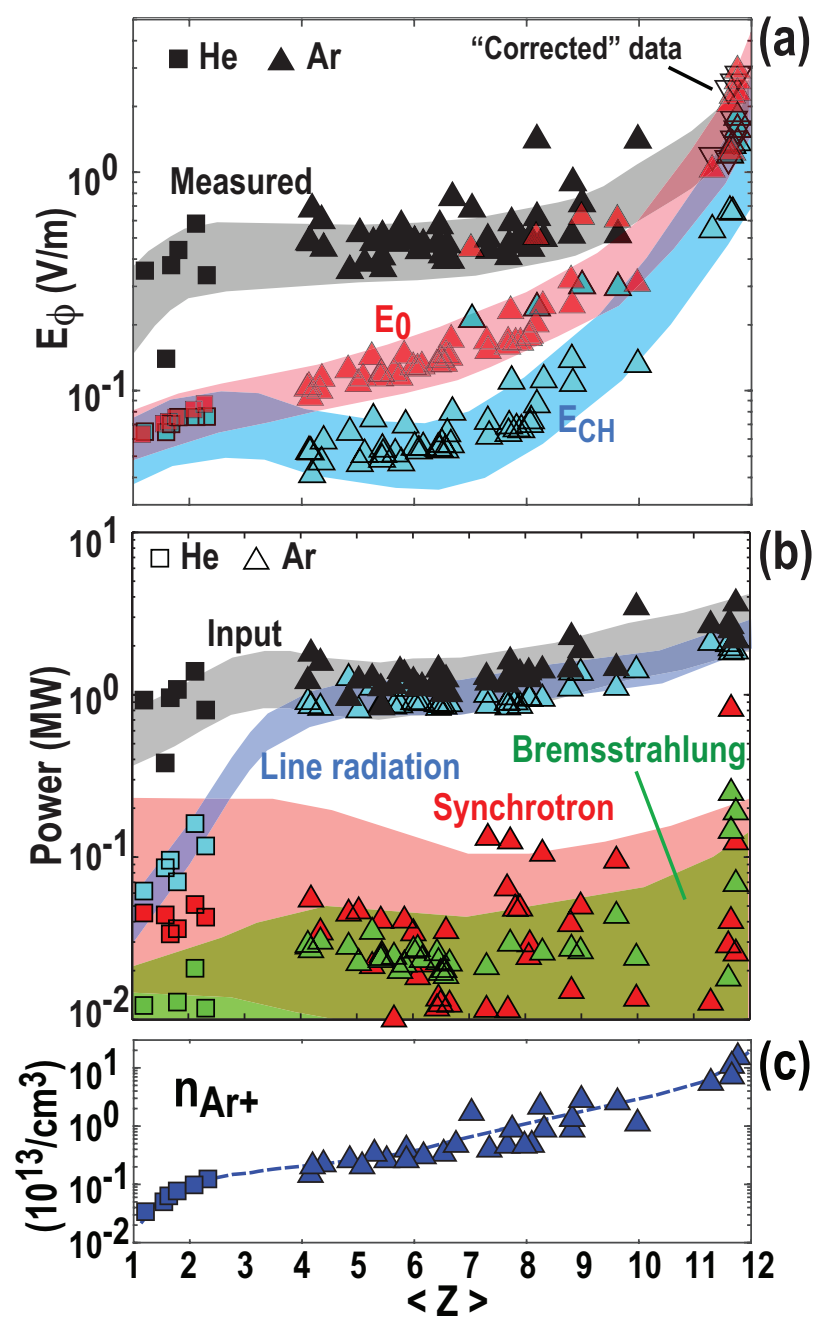

FIG. 35. RE plateau data in DIII-D as a function of mean ion charge $<Z>$ showing (a) toroidal electric field and (b) power balance (c) estimated profile-averaged $\mathrm{Ar}^{+}$density as a function of $<Z>$. Adapted from [210].

Z plateaus, Fig. 35(b). For low-Z plateaus, however, there is an imbalance in Fig. 35(b), suggesting that radial loss does become important, even for well-centered RE plateaus.

Another possible source of the disagreement seen in Fig. 35(a) is errors in the impurity content. Accurate characterization of the background plasma in the RE plateau is important for understanding the collisional dissipation of RE energy and current. In dedicated experiments, the RE plateau is typically created by causing a disruption with rapid Ar injection, either by pellet or massive gas injection (MGI), causing a large RE seed and subsequent RE plateau. As a result, Ar is the dominant impurity in the RE plateau, and collisional drag is 
dominated by the Ar content. In DIII-D, the background plasma was diagnosed with visible and UV spectroscopy, combined with interferometry. Interpretation of the spectroscopic line emission is challenging due to the presence of REs, the very low thermal electron temperature, and the uncertainties in the predicted line emission rates. Factors of 2 uncertainty in the impurity content are therefore possible, and improved data and modeling are desired in this area. In JT-60U, the background plasma temperature was measured to be about $10 \mathrm{eV}$ with Thomson scattering [235]. In DIII-D, it was estimated that the background plasma had significant density $n_{e} \approx 5 \cdot 10^{13} \mathrm{~cm}^{3}$ and was quite cold, with electron temperature $T_{e} \approx 1.5-2 e V$, and with fairly significant $\sim 10-20 \%$ Ar concentration, dominantly in the form of $\mathrm{Ar}^{+}$and $\mathrm{Ar}^{2+}$ [172]. In JET, $T_{e}$ in the RE plateau appears to be somewhat higher, perhaps of order $5 \mathrm{eV}$, as slightly higher Ar charge states are observed with UV spectroscopy [236]. The wide range of observed thermal plasma temperatures $(2-10 \mathrm{eV})$ is not understood yet, but might be related to machine size (machine size could, for example, affect the relative importance of impurity neutrals recycled at the wall and penetrating into the plasma).

Injection of additional impurities into RE plateau has been tried in an attempt to change the RE dissipation rates. This "second injection" is being considered as a method for rapidly dissipating RE in ITER in the event of a RE plateau-forming disruption. To-date there has been no clear evidence that the impurity injection method is important, i.e. either MGI or SPI (shattered pellet injection) of similar neon quantities have shown similar RE plateau current decay times, probably because cryogenic pellets are rapidly vaporized at the RE plateau edge and then behave like gas [237]. Successful assimilation of injected material and the resulting enhancement of the RE current dissipation has been observed in DIII-D [172] and Tore Supra [238]. Massive higher $Z$ injection (Ne or Ar) was seen to increase RE current dissipation in DIII-D, while low $Z$ injection such as He or $\mathrm{D}_{2}$ was observed to decrease dissipation, apparently by purging the initial Ar already present in the RE plateau. However, the mechanism for the apparent Ar purge is not understood yet [210]. The overall effectiveness of this second injection seems to be a combination of competing effects, with the ability of higher $Z$ to more effectively drag on REs competing with the diluting effect of lower $Z$ impurities. For example, the DIII-D experiments indicate that neon is the most effective (in the sense of current dissipation rate per atom injected) gas for RE plateau current dissipation, despite being only moderate $Z$. In JET, massive injection of gases with 
very high $Z$, such as $\mathrm{Kr}$ or $\mathrm{Xe}$, were found to have little effect on $\mathrm{RE}$ dissipation, although some effect was later observed with reduced first injection (smaller initial Ar content in the RE plateau) [239].

\section{RE plateau equilibrium control and vertical stability}

RE plateau control is challenging for the plasma control system because the RE plateau almost always has a smaller current (typically $50 \%$ or less) than the initial pre-disruption plasma, so there is a fairly abrupt (on the disruption time scale $\sim 10 \mathrm{~ms}$ ) drop in the current. Without control system intervention, the uncontrolled RE beam tends to shift to the tokamak center post and then move vertically. Vertical control of the RE plateau has been demonstrated, however, in DIII-D [240] and Tore-Supra [238]. This is achieved with an open-loop push of the plateau off the center post immediately following the disruption and then an Ohmic coil current ramp to a sufficiently large toroidal current to establish good vertical control. With vertical position control established, current control was subsequently demonstrated in DIII-D, with controlled RE current ramps up or down (including ramps down to zero current) 241. RE plateau position control has also been demonstrated recently in FTU [242]. Simulations of RE plateau equilibrium control are not optimistic for ITER: DINA simulations indicate that vertical control can be established only if there is (a) a pre-emptive downward shift of the ITER plasma before the disruption (requiring of order 1 s warning time) and (b) if the initial RE plateau current is sufficiently large ( $\approx 9 \mathrm{MA})$ [11].

\section{E. RE seed formation during TQ}

Understanding RE seed formation during the TQ is important for being able to design disruption mitigation methods in an effort to minimize RE seed formation and to predict the size of the RE plateau in the event that RE seeds are created. Present simulations indicate that the "hot tail" mechanism associated with a very rapid temperature collapse [95] will dominate RE seed formation during mitigated disruptions in ITER [101]. Experimentally, measuring the small RE seed during the TQ is a challenging task, because of the small signal levels relative to the thermal plasma, large spatial anisotropies, and fast time scales.

Some qualitative trends in RE seed formation can be inferred from RE plateau current 
levels measured at the end of the current quench (CQ); this is complicated, however, by the (unknown) prompt loss of REs at the end of the TQ and also the (somewhat unknown) level of avalanche amplification of REs during the CQ. For typical Ar pellet experiment in DIII-D, the final plateau current $I_{r e}(\infty)$ is $200 \mathrm{kA}$ and the initial thermal current $I(0)$ is 1.2 MA. Assuming $l_{i}=2$ and $\sqrt{Z+1} \ln \Lambda=46$ (for Argon dominating the RE drag), we find $I_{r e}(0)=15 \mathrm{kA}$ for the initial RE seed from Eq. (99). Such a seed is much smaller than the initial thermal current of 1.2 MA [230].

JT-60U, ASDEX-U, and JET have observed data supporting a $B \sim 2 \mathrm{~T}$ toroidal field threshold for RE seed formation [206, 235, 243], leading to speculation that excitation of whistler waves could prevent $\mathrm{RE}$ formation below $B=2 \mathrm{~T}$ [158]. However, later experiments in JET [244], KSTAR [245], TEXTOR [246], and J-TEXT [247] found that there is no clear toroidal field threshold, so that $B=2 \mathrm{~T}$ is not a prerequisite for $\mathrm{RE}$ formation. Nevertheless, a robust trend is observed across all machines that higher toroidal magnetic field does elevate the RE plateau levels.

Another robust trend across experiments is that higher- $Z$ injection shutdown creates more RE seeds, with low $Z$ MGI (He, $\mathrm{D}_{2}$ ) rarely creating significant RE seeds [244, 248]; this is consistent with hot tail seed formation, as higher $\mathrm{Z}$ impurities will radiate more strongly, causing a more rapid TQ.

Electron cyclotron emission (ECE) during the TQ is very sensitive to non-thermal electrons and can clearly see RE seed formation during the TQ. The RE seed emission can be partially isolated from the thermal ECE emission as being emission apparently from minor radii outside the plasma column (due to the relativistic downshift of the gyro-frequency) [85]. The TQ non-thermal ECE does not appear to be a reliable indicator of RE seed formation, however, as it is clearly seen in some RE plateau-forming shots and not in others, possibly due to the complicated impurity mixing that causes ECE cutoff in some disruptions and not in others. As a result, non-thermal ECE emission has not yet been interpreted quantitatively to determine RE seed levels.

Ar pellet ablation rates were used in DIII-D to estimate RE seed populations [85]. This was done using the same Ar pellet which is used to create the REs in the first place, i.e. Ar ablated from the pellet, caused the TQ and formed REs, after which the REs vaporized the remaining Ar pellet much more rapidly than the thermal plasma could. The resulting estimates of RE seed current had very large error bars (of order 10×) but roughly 


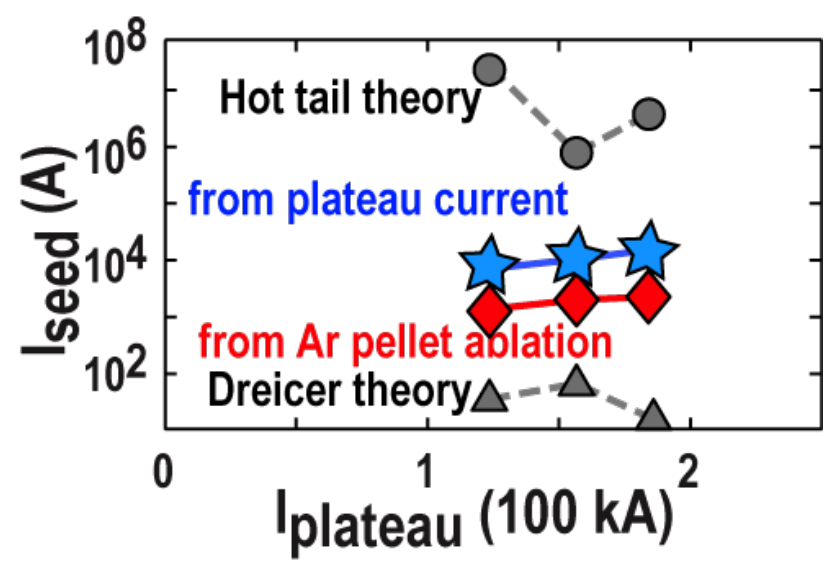

FIG. 36. RE seed estimated in DIII-D experiments from Ar pellet injection (red diamonds), from the initial RE plateau current assuming a $20 \times$ avalanche gain (blue stars), from Dreicer theory (gray diamonds), and from hot tail model [95] (gray circles). Figure adopted from Ref. [85].

consistent with $10 \mathrm{kA}$ RE seed currents. Estimates of Dreicer and hot tail seed terms for the experiments have shown that the Dreicer model [2] under-estimated the measured RE seed level by of order $100 \times$, while the hot tail model [95] over-estimated the measurement by of order $100 \times$, as shown in Fig. 36. In this comparison, the Dreicer seed was calculated using the Z-dependent version of the Dreicer formula [40]. The hot tail seed is calculated by integrating Eq. (4) of Ref. [95] over velocities using the velocity distribution function for very rapid temperature decay, Eq. (9) of Ref. [95]. More recent kinetic modeling of the seed formation (see Section VD and Ref. [89]) predicts seed currents that are smaller than the measurements if one assumes a toroidally symmetric impurity distribution. Yet, this work also predicts that toroidal asymmetries should enhance the seed significantly. Further modeling work is clearly necessary for better prediction of the disruption RE seed.

\section{F. RE prompt loss due to magnetic perturbations}

RE "prompt loss" due to magnetic perturbations is often observed in the form of HXR and SXR spikes at the end of the TQ, associated with a sudden loss of seed REs to the wall. 3D MHD simulations relate the TQ and the prompt RE loss to magnetic island overlap and destruction of good magnetic surfaces. [70, 249, 250]. Experimentally, overall trends in RE seed formation and confinement have been studied in various tokamaks [25, 206, 251]. 
Details of RE loss during the TQ is expected to be quite complicated though due to coexistence of good magnetic surfaces, islands, and ergodic regions [25]. Examples of that are shown in Figs. 4 and 5. Modeling transport across this complex magnetic topology is challenging, since the RE transport is expected to be a mixture of convection and diffusion, as described in Sections II B and IV B and in Ref. 224. As discussed there, RE transport in the TQ is not generally diffusive, can be strongly energy-dependent, and depends sensitively on the degree to which neighboring resonances overlap. Additionally, interpretation of experiments has been complicated by the lack of a good RE seed diagnostic and also by interlinking of different experimental parameters, making clear parameter scans and conclusions elusive. As discussed in Section V, predicting RE seed levels from first principles is extremely challenging, so the lack of an in-situ RE seed measurement makes interpretation of plateau RE current levels difficult. For example, lowering the initial plasma $n_{e}$ generally results in higher plateau RE current levels, but it is not certain if this is due to an increased initial hot tail seed from increasing $T_{e}$ or an increased surviving RE seed due to decreased TQ MHD prompt loss [247]. In diverted DIII-D plasmas, the SXR localization of the wall flash was shown to correspond to the divertor outer leg, consistent with the expected current direction of the shot [174]. This understanding is consistent with experiments showing larger RE loss levels in plasmas with elongated magnetic surfaces, which are predicted to be more prone to island overlap than circular discharges [174]. Prompt loss is also predicted to be larger in smaller tokamaks and could explain the absence of post-disruption RE plateaus in some of them, e.g. C-MOD. TEXTOR observed improved confinement of REs inside an $m=1$ island structure [252, while J-TEXT observed improved RE confinement inside an $m=2$ island [253]. Comparisons of RE loss times in LT-3, TM-3, ST, PLT, Ormak, and JT-60U suggested that REs are lost more rapidly than thermal electrons in the presence of macro-scale magnetic turbulence but more slowly than thermal electrons in the presence of micro-scale magnetic turbulence; this was attributed to perturbation averaging over the large RE drift orbits [25].

Many efforts have also been made to modify the TQ with externally applied nonaxisymmetric magnetic fields (RMPs) in order to affect RE prompt loss levels. In TEXTOR, an applied $n=3$ field had a clear affect on final RE levels [35]. In DIII-D, less effect was seen: both $n=1$ and $n=3$ were attempted and some effect may have been observed, but limited statistics made clear conclusions difficult to achieve [254]. Similar experiments on 
RMP effects on RE prompt loss were tried in JET, but no clear effect was seen. Experiments in ASDEX-U also applied a fixed $n=1$ field on the low field side of the torus, but with a pitch that was varied from shot to shot: a clear effect on post-TQ RE levels was seen at a pitch that was roughly field aligned at the outer midplane [255].

In addition to the TQ-related prompt loss, it was hypothesized that a second prompt loss event might be created during the CQ by additional rapid impurity injection. The additional impurities could destabilize MHD due to current profile shrinking, thus enhancing RE loss to the wall sufficiently to counteract the runaway avalanche [256]. Experiments attempting to demonstrate this were performed at Tore-Supra by using burst disk MGI to fire neon into the CQ of plasmas shut down by a first injection of Ar. Some MHD events (current flattening) and RE loss events were seen during the CQ, but no clear correlation with the presence or absence of the mid-CQ neon injection was observed [257]. As a possible explanation of this result, it was suggested that ionization of the neutral gas precludes delivery of impurities into the current channel, even in the absence of RE current [258].

\section{G. Collisional suppression of CQ RE avalanche}

In principle, sufficient impurities present in the plasma during the CQ can suppress the RE avalanche and prevent a RE plateau from forming. Many experiments have attempted to inject greater and greater quantities of impurities into tokamaks during or before disruptions to attempt to achieve this "critical density" $n_{\text {crit }}$ of free + bound electrons. The critical density increases with toroidal electric field, which itself increases as more material is injected and is therefore something of a moving target. Experiments aimed at achieving $n_{\text {crit }}$ were pursued, for example, in TEXTOR [259], ASDEX-U [243], Tore Supra [260], MAST [261], DIII-D [262], and JET [244]. A variety of injection methods were attempted, MGI valves [263], shattered pellets (SPI) [264], rupture disk gas injection [265], and large shell pellets [230]. Overall, no injection method has come close to achieving $n_{\text {crit }}$, with best results giving of order $20 \% n_{\text {crit }}$ [230]. One of the main difficulties appears to be that the amount of impurities required to shut down the plasma and cause the TQ is far less than $n_{\text {crit }}$ (often of order $1 \%$ or less) so the plasma tends to shut down before most of the impurities arrive or are assimilated. Coupled zero-dimensional current filament simulations including basic atomic physics (ionization, recombination, radiation, etc) indicate that achieving $n_{\text {crit }}$ is 
almost impossible with MGI but instead will require some form of "ideal" instantaneous deposition such as exploding shell pellets. It also follows from these simulations that even if $n_{\text {crit }}$ could somehow be achieved with a perfect impurity deposition system, the CQ duration in ITER would be too short, giving unacceptably high induced vessel forces [11].

\section{H. RE energy deposition into the wall}

If a RE plateau moves into plasma-facing components, the REs are rapidly lost into the wall, potentially damaging the components [266]. Understanding this process is important for designing components to resist RE-wall strikes and for designing an effective mitigation system to minimize RE wall damage [267].

Data on the wetted area during the RE final loss is sparse. IR images have been captured in JET, indicating multiple strike points on tile leading edges, with spot sizes of order $10 \mathrm{~cm}$ [268]. IR imaging in DIII-D has seen similar wetted area spot sizes [207]. A toroidal array of HXR sensors in DIII-D indicated a strong toroidal variation in the HXR emission [174]. The toroidal phase of the HXR emission was shown not to be random, but exhibited preferred phases, suggesting that mode locking to field errors or structures occurs during the final loss [207]. The underlying physics of this localized heat deposition is not well understood at present, but may be due to a complicated interplay of magnetic topology (MHD) and kinetic effects (drift orbits).

HXR data shows that the deposition of RE energy into the wall is not smooth in time, but occurs in sharp, narrow spikes [207]. The duration of the spikes (as short as $10 \mu \mathrm{s}$ ), Fig. 37(a), is long compared to the RE toroidal transit time (of order $30 \mathrm{~ns}$ ), but is normally very short compared with the wall poloidal field penetration time (of order $8 \mathrm{~ms}$ in DIII-D). The short duration of the spikes does not look consistent with current channel motion into the wall (scraping off). In Ref. [36], it was suggested that such spikes could result from occasional percolations of the field lines through good magnetic surfaces that surround an ergodic annulus. This would bring some RE electrons from the annulus to the wall. The spike spacing can be of order $0.1-1 \mathrm{~ms}$, Fig. 37(b), while the total duration of the final loss (integrated over the narrow loss spikes) is typically of order several ms; this duration appears to be roughly consistent with the avalanche time scale, indicating that perhaps the final loss dynamic involves periodic, rapid loss from ergodic regions, followed by flux surface 


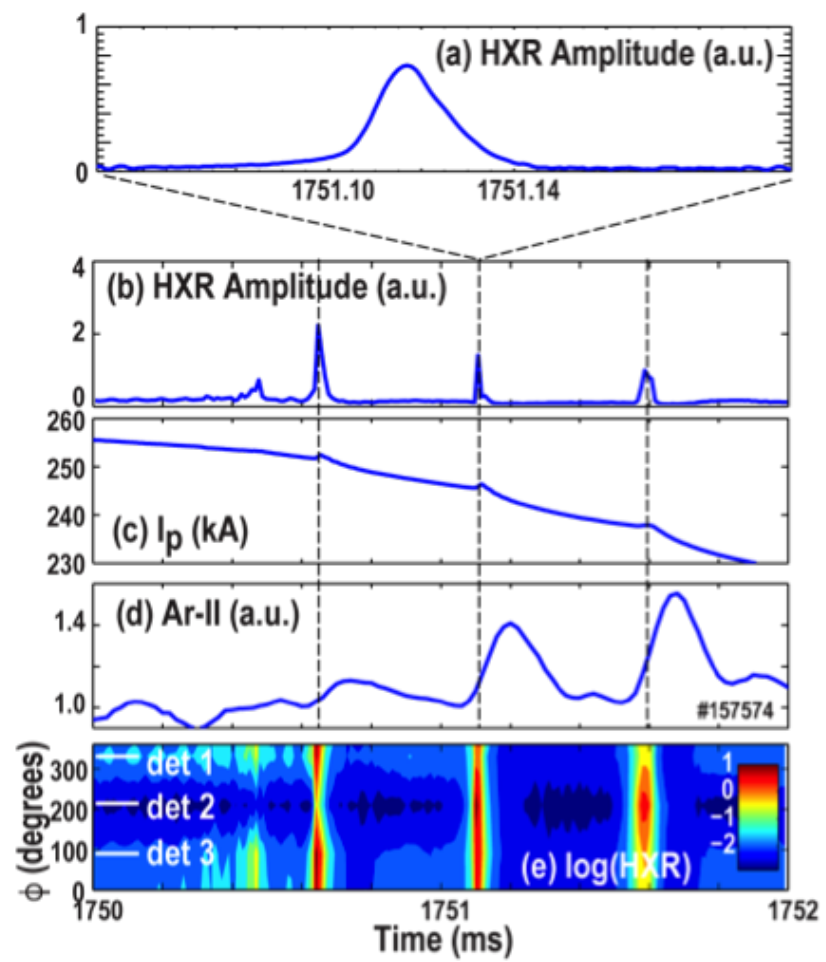

FIG. 37. Time traces during the final loss of a RE plateau to the wall of DIII-D showing: (a) a single HXR spike showing narrow spike width, (b) three spikes showing spike frequency, (c) plasma current, (d) Ar-II line emission, and (e) toroidal contours of HXR emission showing toroidal structure. Adapted from Ref. [207].

healing and RE current profile reforming due to avalanche gain [207]. A very slight rise of the plasma current is observed during the HXR spikes, Fig. 37(c), indicating a slight broadening of the current profile. A slower rise in line emission, Fig. 37(d), between spikes is consistent with the loss of REs from an annulus and a resulting temporary rise in local Ohmic current. The toroidal structure of the HXR spikes indicates significant toroidal peaking which can change phase spike-spike, Fig. 37(e).

The total energy content in the RE plateau is thought to be dominantly magnetic in present experiments. This is expected to be even more the case in ITER, since $W_{k i n} \sim I_{P}$ but $W_{\text {mag }} \sim I_{P}^{2}$. The conversion of magnetic to kinetic energy during the final loss strike is therefore expected to be the dominant source of wall-damaging RE-wall strikes in ITER. A OD coupled-circuit modeling indicates that longer RE loss times at the wall will cause greater conversion of magnetic to kinetic energy, while fast loss $\tau_{\text {Loss }}<\tau_{\text {Wall }}, \tau_{\Omega}$ (where $\tau_{\Omega}$ is the background plasma resistive time scale) will result in the RE current dominantly being 
converted into Ohmic current and vessel current [269]. This overall trend was confirmed with comparison to JET, DIII-D, and FTU data [269]. It is essential that the RE plateau current can be converted into wall current (and then dissipated resistively in the wall) for sufficiently fast loss. This would prevent conversion of the entire poloidal magnetic field energy into REs. 


\section{ITER DISRUPTION MITIGATION STRATEGY}

Disruption mitigation in ITER must be the last resort when disruption prevention and active attempts for disruption avoidance have been unsuccessful. The mitigation strategy in ITER relies on massive injection of impurities to dissipate thermal and magnetic energy through line radiation while preventing formation of runaway electrons. The present baseline concept of the disruption mitigation system (DMS) is based on the shattered pellet injection (SPI) technology [270]. This technology involves acceleration of large cryogenic pellets that break into smaller fragments at the end of the delivery tube. The fragments enter the plasma, ablate and eventually evaporate on their way through the plasma.

\section{A. Rationale for choosing SPI}

The SPI technology has been chosen for ITER for two main reasons. The first reason is the more efficient delivery of the material to the plasma compared to Massive Gas Injection (MGI), which is the widely used technique for disruption mitigation. In ITER, the injection valve is required to be located outside the port plug, due to radiation load from gamma rays and neutrons and due to the need for maintenance. Simulations for ITER have shown that the gas flow at the end of the delivery tube will be stretched significantly over time due to the significant length of the tube. As the gas streams into the vacuum vessel, the plasma will be cooled down from the edge. When this cold front arrives at the $q=2$ surface, the thermal quench will set in. However, at that time, only a small fraction of the gas stored in the MGI valve will have reached the plasma, e.g. for neon injection only about $10 \%$ [271]. Most of the gas will arrive after the thermal quench and cannot contribute to mitigating the thermal loads and avoiding runaway formation. In contrast to this, SPI will deliver the total quantity almost simultaneously to the plasma. The second reason to choose SPI for ITER is the envisioned deep penetration of the material inside the plasma. Simulations with the 3D MHD code JOREK show that SPI with deuterium indeed can lead to a much higher density rise compared to MGI [272]. More recent simulations with the same code for neon SPI show, however, an $n=0$ current contraction as the cold front moves inward with the SPI fragments [272]. This behaviour is similar to MGI. But, in contrast to MGI for

which the density rise in the plasma centre relies solely on MHD mixing, SPI is expected to 
raise the density also due to direct ablation of fragments that will continue flying towards the plasma centre during the thermal quench. The assimilation of the injected material in the pre-TQ phase depends on the fragment size, the ablation rate and the velocity of the fragments. The final distribution of impurities and electron density will then depend also on how the MHD modes will redistribute the ablated material. Optimising the fragment size distribution to ensure sufficient ablation is critical. This means ensuring sufficiently deep penetration of the fragments while avoiding that too many fragments cross the plasma and strike the opposite wall. NIMROD simulations for SPI in ITER show that the assimilation efficiency depends strongly on the initial fragment sizes [273]. Note that for late injection into an existing runaway beam, SPI is not expected to be more efficient than MGI (see Section $\mathrm{XC}$.

\section{B. Disruption Mitigation Requirements for ITER}

The design of the DMS has to ensure that the following requirements are met.

\section{Provide sufficient radiation to reduce conductive energy losses during the thermal quench}

Thermal quench mitigation aims at radiating a large fraction of the stored thermal energy before it is lost to plasma facing components through heat conduction. Studies show that the heat fluxes to the inner divertor are the most critical and it is expected that surface melting can occur on the target plates at about $20 \mathrm{MJ}$. Therefore for $E_{t h}>20 \mathrm{MJ}$ the required fraction of radiated energy is $f_{r a d}=1-20 \mathrm{MJ} / E_{t h}$.

\section{Dissipate the magnetic energy through radiation}

The dissipation of magnetic energy aims on a) avoiding excessive heat loads on plasma facing components and b) controlling electro-magnetic loads on tokamak components.

The heat load reduction goes along with the reduction in halo current by increasing radiation levels and thus increasing resistivity. These heat loads are presently under assessment for ITER. Initial studies showed that melting of the first wall beryllium panels can be expected above about $7 \mathrm{MA}$ of plasma current. In that case the required radiation fraction 
for currents above $7 \mathrm{MA}$ is $f_{\text {rad }}=1-\left(7 \mathrm{MA} / I_{P}\right)^{2}$.

The constraints for the timescale on which the magnetic energy must be dissipated are exclusively determined by the electro-magnetic forces. The faster the current quench, the higher the eddy currents are, caused by $d B / d t$. The most critical components in this respect are the blanket modules [274]. The design of these components needs to take into account fatigue lifetime and possible plastic deformation. The ITER load specifications limit the linear current quench duration (from initial $15 \mathrm{MA}$ to zero) to $>36 \mathrm{~ms}$ for 400 events and to $>50 \mathrm{~ms}$ for 2600 events. The lower limit has been determined from an experimental database that predicted the minimum current quench time in ITER to be $36 \mathrm{~ms}$ [275]. MGI experiments performed since then indicate that faster current quenches can be expected for high quantities of neon or argon. This is confirmed by simulations with the code DINA [138]. Simulations with the free boundary equilibrium code DINA have shown that current quench acceleration to below about $150 \mathrm{~ms}$ is sufficient for the required reduction in halo currents [276]. However, this current quench time is indicative only and the required current quench time and related injection quantity will be validated during the commissioning of the DMS in ITER. It has to be noted here that the plasma always becomes vertically unstable if the current decays with a rate faster than about $0.3 \mathrm{MA} / \mathrm{s}$. The halo current reduction depends significantly on the equilibrium at the start of the current quench. The less displaced the plasma is at this time, the lower the resulting halo current.

\section{Provide a radiation distribution homogenous enough to prevent first wall melting}

Although allowable levels of radiation peaking are predictable, the physics understanding is not yet mature enough to conclude how a sufficiently low peaking can be guaranteed. Work on MGI has shown how the peaking depends on injection location and MHD activity during the thermal quench [277, 278] in this case. However, with SPI, the physics basis is not mature enough to confirm that the presently foreseen injection scheme for ITER is appropriate.

Taking the melt limit for stainless steel, which is the first wall material of the diagnostic ports and thus also for the ports of the DMS, the critical energy and peaking factor can be

estimated. With a surface area of $700 \mathrm{~m}^{2}$ and a duration of the mitigated thermal quench of $1 m s$ the critical overall peaking factor PF and thermal energy $E_{t h}$ is $P F \times E_{t h}<288$ MJ. 


\section{Prevent runaway formation during all phases of the disruption}

Any action of the DMS to mitigate the thermal and electro-magnetic loads has to ensure that runaway electron formation is excluded. Presently, densification through injection of deuterium is foreseen for this purpose. This is an active field of research and this scheme is not yet confirmed as effective for ITER (see XIG).

\section{Provide fast dissipation of the runaway electron energy}

In case of accidental generation of runaway electrons during a disruption, collisional dissipation of their energy using high-Z impurities is presently envisaged. The effectiveness of this scheme is discussed further below (XIG).

\section{Injection quantities to respect upper limits from vacuum handling and deflagration risk}

The upper limits on injected quantities are determined by gas handling capabilities and deflagration limits: $1.1 \cdot 10^{25}$ molecules of $\mathrm{D}_{2}, 2.2 \cdot 10^{25}$ atoms of argon or neon, and $9 \cdot 10^{24}$ atoms of helium. These numbers include also the gas required to accelerate the pellets.

\section{Mitigation targets for runaway electrons}

To quantify the targets for suppressing or mitigating runaway electrons, it is required to determine the maximum current at which melting of plasma facing components can still be excluded. Simulations of runaway electron energy deposition in ITER have been performed for various impact energies using the codes ENDEP for calculating the energy deposition in the bulk material and MEMOS for calculating the resulting temperatures and material erosion [279]. In contrast to the heat flux deposition during the thermal quench, the impacted area from runaway electrons is very small as seen for example in JET [280]. Consequently, the critical energy above which melting occurs is very low, in these simulations around 0.3 MJ deposited on one of the roofs of the first wall panels (32 roofs are in a toroidal row in the impact area on top of the vessel). Considering, that a runaway current of $10 \mathrm{MA}$ could deposit an energy as high as $300 \mathrm{MJ}$ [269], this melt limit translates to a runaway current of about $300 \mathrm{kA}$ should all the energy be deposited on one panel or about 1.9 MA should 


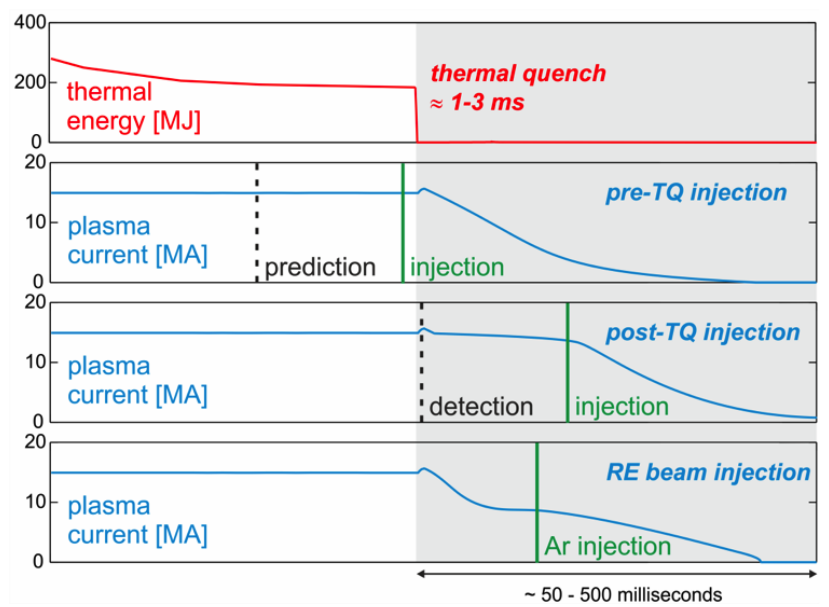

FIG. 38. Schematic representation of the different injection scenarios for the mitigation of the thermal quench, the current quench and for runaway energy dissipation. The delay between the prediction/detection of a disruption to the actual arrival of the gas is in the most optimistic case about $30 \mathrm{~ms}$.

the energy be distributed uniformly over all 36 roofs. Narrowing down the critical current would - besides code validation - require better understanding of the driving mechanism for toroidally asymmetric energy deposition as seen for example in JET [268].

\section{ITER DMS injection scenario}

The ITER DMS will be activated through a disruption predictor that aims to predict a forthcoming disruption within the reaction time of the DMS. In case prediction is successful, the DMS will be triggered early enough to initiate the thermal quench of a mitigated disruption. Since disruption prediction cannot be $100 \%$ reliable, a second algorithm will be implemented that is able to detect that a thermal quench has already occurred. Naturally, in this case, mitigation of the thermal quench is not possible anymore, but mitigation of the current quench can still be achieved and has to be achieved with high reliability to avoid melting of the first wall from conductive loss of magnetic energy, if line radiation levels are low. These two scenarios are depicted in Figure 38.

In the first scenario for which prediction has been successful, the injection from the DMS aims to radiate the thermal energy on the timescale of the thermal quench (requirement XI B 1]. This timescale is expected to be of the order of 1-3 milliseconds in ITER [281]. For 
this purpose, the DMS will inject neon atoms. From simulations with the code DINA, it is

found that (requirement XIB 2) will be fulfilled for neon quantities of up to a maximum of $5 \cdot 10^{22}$ atoms [282]. This requirement will define the maximum quantity of neon that can be injected prior to the thermal quench. In addition to neon, deuterium will be injected simultaneously to raise the density and discourage runaway electron formation. Deuterium is a weak radiator and thus can be injected in large quantities without accelerating the current quench much. However, the impact from raising the electron density on the radiated power remains to be studies. The amount of deuterium is restricted to $10^{25} \mathrm{D}_{2}$ molecules (requirement XIB 6).

The second scenario will inject neon into the early current quench to increase the radiative dissipation of the magnetic energy. Similar quantities like in the first scenario will have to be used to fulfil (requirement XIB 2). In this scenario, the DMS cannot prevent runaway seed generation from the hot tail mechanism. In contrast with the first scenario, suppression of runaway formation in this second scenario has not been studied at all so far.

The two scenarios described above aim to mitigate the thermal and the current quench while avoiding runaway formation. In addition, a second delayed injection is envisioned in the present ITER mitigation scheme. It will deliver argon atoms to provide enough argon in the plasma to dissipate the runaway energy through collisions and line radiation during vertical movement of the runaway beam that formed despite the efforts to suppress runaway formation. This injection is pre-emptive and must be delayed to avoid excessive electro-magnetic loads on the blanket modules from too fast a current quench (requirement XI B 2).

\section{E. ITER DMS layout}

The design and layout of the DMS is still under development and a plan for design validation through dedicated experiments, modelling and theory efforts and technology development has been established. The design present layout has three injection locations on top of the machine that will be used for post-TQ injection and three injection locations in the equatorial plane that will allow the injection of mixtures of neon and deuterium for thermal quench mitigation and runaway electron avoidance as well as the injection of argon pellets for runaway electron energy dissipation [283]. A total of up to 32 pellets can be 
injected from the equatorial ports with a diameter of $28 \mathrm{~mm}$ and length to diameter ratio of $L / D=2$. Each of these pellets can deliver either $9 \times 10^{23}$ argon atoms or $2 \times 10^{24}$ deuterium atoms mixed with minor quantities of neon of the order of $10^{22}$ atoms. The injection is distributed over three locations in the toroidal direction to comply with requirement XI B 3. However, the effectiveness of this strategy is still to be assessed through appropriate tokamak experiments. The velocities that can be achieved for these large pellets are expected to be of the order of $150 \mathrm{~m} / \mathrm{s}$. The pellets for post-thermal quench injection from the upper ports are up to $20 \mathrm{~mm}$ in diameter (exact value to be determined) with a length to diameter ratio of $L / D=1.5$. These pellets will be shattered to very fine fragments or to mainly gas to ensure high assimilation in the relatively cold post-thermal quench plasma. Higher velocities can be achieved for these pellets, of up to $400 \mathrm{~m} / \mathrm{s}$, minimising the delay from trigger to arrival of the material in the plasma.

\section{F. Runaway electron diagnostics in ITER}

The main diagnostic for runaway electron detection in ITER is the hard X-ray monitor (HXRM) 284]. It will be available from first plasma until the end of the non-nuclear phase of ITER operation after which it will lose its function due to the high neutron flux. The HXRM will be essential to detect possible runaway formation during the commissioning of the DMS. For this purpose, it is essential that the detection limit for confined runaway electrons is low to ensure runaway electron currents that would cause damage of the first wall (see XIC). Also for post-event analysis in case of an accidental formation of a runaway beam, data from the HXRM will be an important ingredient. Here, the de-confined runaway electrons will create high levels of hard X-rays when interacting with the in-vessel structure. Therefore, the dynamical range has to be very wide and ranges from a few kA to 10 MA. For energy resolved measurements, the dynamic range will be more restricted. The HXRM is suited to detect runaway electrons during disruptions and could be used as a trigger in mitigation schemes. However, as stated above, its availability is restricted to the non-active phase of ITER operation. Two other diagnostics that could be used for runaway electron detection during the nuclear phase of operation are ECE [285] and the radial gamma-ray spectrometer [173]. The latter will have a detection limit of $40 \mathrm{kA}$ and time resolution of about $10 \mathrm{~ms}$ and can thus be used for early detection of runaway electrons (note that this 
diagnostic is not fully credited yet). Additionally, the IR and visible cameras [286] can detect synchrotron radiation if the runaway density and energy is high enough. However, due to the high detection threshold in energy and the expected background emission, these cameras are more likely to contribute to post-event analysis rather than to generating a trigger for the DMS.

\section{G. Needs to validate the DMS design and strategy}

The main risks and gaps in physics and technology in view of runaway electrons are briefly summarised in the following.

\section{Avoidance of runaway electron formation}

Achieving high densities for disruption avoidance during the thermal quench requires the simultaneous injection of several pellets. For the assimilated quantities as predicted for example in [101] already 6 pellets with $28 \mathrm{~mm}$ diameter would be required if $100 \%$ assimilation efficiency is assumed. On the technology side, it remains to be shown that simultaneous injection with sufficiently small jitter in injection timing is possible and that the efficiency (namely the radiation and the density rise) increases linearly with the number of injected pellets. On the physics side, there are significant gaps in understanding runaway formation during the thermal quench, which makes it difficult to claim that the present schemes are able to effectively suppress runaway formation. This is mainly due to the still fragmentary understanding of the thermal quench. The challenge is to develop a complete description of the thermal quench with all its essential elements such as cooling down of the initially hot plasma, MHD activity, and atomic physics that governs radiation. First attempts to assess efficiency of deuterium injection for runaway suppression were based on the early version [95] of the hot tail mechanism and predicted that assimilated quantities of the order of $10^{24}$ deuterium atoms could be sufficient to suppress the hot tail runaway seed as well as the additional seed mechanisms during the DT phase of operation (Compton scattering and tritium decay) [101]. A more consistent approach that calculates the duration of the ther-

mal quench from the radiation balance shows that deuterium injection could enhance the generation of seed runaway electrons at high electron temperatures [115]. However, when 
taking into account the current quench phase, these simulations also qualitatively confirm that adding deuterium can reduce the final runaway electron population. Further experimental and theoretical efforts are required to assess effectiveness of this scheme and to better understand the mechanisms. Another issue that requires attention is the penetration and ablation process of the pellet fragments. Initial simulations with the code JOREK show that, although deeper penetration could be expected if the fragment sizes are optimised and adopted to the target plasma density and temperature, the spatial distribution of the resulting plasma density rise is strongly affected by the MHD activity that drives the thermal quench [272]. Since a density rise over the entire cross-section is a prerequisite for reliable runaway suppression, this issue needs more attention in modelling and experiments.

\section{Runaway electron energy dissipation}

The effectiveness of the second injection of high-Z gas into a runaway beam has been tested in several tokamaks [210, 268, 287, 288, but large uncertainties remain as to whether this scheme will be effective in ITER. Saturation in the achievable dissipation rates have been seen in experiments (e.g. [289]) that is likely caused by long transport times to bring the impurities into the background plasma of the runaway beam. First attempts to inject impurities with SPI into a runaway beam confirm the expectation that also the ice fragments are not penetrating the beam since similar dissipation rates were achieved as for MGI [237]. It was also shown that due to the high conductivity of the ITER vacuum vessel, the vertical position of the beam is a function of the runaway current [144]. Therefore, the faster the current is decaying, the faster the runaway beam will move vertically. Simulations with the code DINA including a simplified runaway model show that the scraping-off of the runaway beam during the vertical displacement will cause an increase in the electric field that will slow down the runaway decay [132]. Another consequence of the vertical displacement is that the plasma will become kink unstable while there is still a finite runaway current.

\section{H. Other runaway electron mitigation techniques}

Other means that have been discussed in the literature to mitigate runaway electrons are externally applied perturbation fields and runaway beam control to allow mitigation 
techniques that require more time, like reversed loop voltage or collisional dissipation at lower impurity levels. Magnetic perturbations can be imposed by external coils, and this has been shown to suppress the formation of a RE beam above a certain amplitude threshold. Modelling for ITER has shown that the perturbation imposed by the in-vessel ELM mitigation coils can create a stochastic layer at the plasma edge only and is therefore insufficient for RE suppression [34]. Measurements at TEXTOR give evidence that intrinsic broad band magnetic fluctuations can prevent RE beam formation [290]. Similar findings are reported from J-TEXT [247]. More recently, it was observed at DIII-D that the absence of a runaway current plateau is correlated with the existence of Alfvén-like instabilities [289]. It is also discussed whether active methods can be applied to excite kinetic instabilities to aid runaway energy dissipation. Today, it remains unclear whether the interaction between runaway electrons and waves can be effectively used for disruption mitigation.

Active destabilisation of MHD modes during the current quench had also been considered a possible method to deconfine seed runaway electrons before a high current runaway beam is formed. It was proposed to inject impurities during the current quench phase in order to destabilise MHD modes by increasing resistivity locally. For this purpose a special injector had been built using bursting disks to ensure fast gas delivery and short pulses [265]. This injector has been implemented at Tore Supra to allow injection during the current quench. Despite the deep penetration of the gas jet, no impact on the current decay or MHD activity was detected. This result is confirmed by experiments on ASDEX-Upgrade using a MGI valve to inject into the CQ. Although MHD events took place, they were not correlated with gas injection. There are two possible reasons for the absence of MHD destabilisation: a) the local change in resistivity is too small compared to the already highly resistive, low temperature current quench plasma; b) an electrical breakdown across the gas jet terminates high resistivity [258]. MHD modes that develop during the runaway beam phase have been observed experimentally and predicted numerically for ITER [133] and could lead to high heat loads on first wall components of ITER. Additionally, frequent reoccurrence of such events during the plateau phase can also facilitate high magnetic to kinetic energy conversion factors [269, 291]. It has been discussed whether impurity injection into the runaway beam aiming at collisional energy dissipation could trigger such MHD events [12]. So far it was not reported from experiments that MHD events are correlated with injections during the runaway plateau phase. This is also true for experiments at JET for which the edge safety 
factor is in the same range as can be expected for ITER [268].

Active position control of runaway beams in ITER has been studied for disruptions at 15 MA and 7.5 MA [292]. These simulations with the code DINA show that position control in ITER is only possible under very limited conditions, due to a relative weakness of the ITER control system with respect to the plasma current. Early warning of a forthcoming disruption would be required for shifting the current barycentre towards the neutral point. The current drop during the current quench must be below one third of the initial plasma current, therefore the runaway current must be above $10 \mathrm{MA}$ in case of $15 \mathrm{MA}$ disruptions. Finally, the subsequent decay of runaway current must be in the range of 0.5-1.0 MA/s. Commissioning of such a control scheme is not feasible since it would involve generating significant runaway currents for testing. Upgrading the vertical position system to allow runaway beam control for a broader parameter space is not possible with the ITER design. The current in the in-vessel vertical stabilisation coils is limited because of space restrictions. It will not be possible to accommodate a larger coil in the space between the blanket modules and the vacuum vessel. However, technical feasibility runaway beam controllability should be assessed when designing reactor-grade tokamaks. 


\section{SUMMARY}

Runaway electrons are interesting and hazardous. This review discusses both aspects and what has been learned about them from the last two decades of the theoretical and experimental studies in anticipation of ITER operation. The paper first revisits fast electron motion in the equilibrium and perturbed magnetic field of a tokamak and discusses collisional processes involved in the runaway kinetics. Two processes have received particular attention in recent literature: synchrotron losses and elastic scattering of the electrons by partially ionized heavy impurities. Taken together, they limit energy gain of the runaway electrons. The impurities also govern rapid cooling of the bulk electrons via line radiation. This appears to be the dominant mechanism of the thermal quench that triggers runaway production. An alternative mechanism is the anomalous electron heat loss to the wall along the stochastic magnetic field lines, but this loss channel is more difficult to ascertain because of its sensitivity to the level and spatial scales of the magnetic perturbations. Incomplete present understanding of the thermal quench still remains a major obstacle for predictive modeling of the runaway build-up. In addition to the unsettled debates regarding relative roles of impurities and magnetic fluctuations, there is a need for first-principle description of impurity transport from the wall into the plasma core as well as for assimilation of impurities introduced via pellet injection. In particular, the developing theory should address the expected significant role of nonuniformity of the injected impurities. There have been several essential findings in the theoretical work of recent years. A combination of pitchangle scattering and synchrotron radiation has been found to raise the critical field for runaway avalanche above the Connor-Hastie field. It has been shown that the distribution function of the runaway electrons tends to be non-monotonic in energy. Heavy impurities (irrespective of their ionization degree) have been shown to enhance elastic scattering of the runaway electrons significantly under plausible runaway mitigation scenarios in ITER. A hot tail mechanism of primary runaway production has been proposed. Self-consistent kinetic modeling of this mechanism demonstrates prompt conversion of the plasma current into a sub-MeV runaway current for heavy injection of impurities. Thresholds for the runawaydriven micro-instabilities have been quantified and initial quasilinear simulations performed in support of the ongoing experiments.

Several numerical codes now enable experimentally relevant test particle modeling of run- 
away electron transport and losses, but a self-consistent description of the runaway impact on magnetic flux surface stochastization is still a challenging task to be completed. The time-scale of avalanche-controlled runaway current decay in ITER is shown to be comparable to the time-scale of vertical displacement events (VDE), which indicates the need of self-consistent analysis of the VDEs and the runaway avalanche.

Experimental studies of the runaway electrons now become significantly more detailed due to continuous improvements in diagnostics. Runaway electrons span very broad energy range from several $\mathrm{keV}$ to nearly a hundred $\mathrm{MeV}$, and standard tokamak diagnostics were not optimized for that. More specialized measurement methods and analysis techniques are now developing, such as synchrotron imaging. Multi-machine comparison shows that runaway electrons become detectable in the density ramp-down experiments later than expected, i.e., when the driving electric field is already $5-10 \times$ times larger than the Connor-Hastie field. This is conceivably due to the weakness of the Dreicer source that is unable to create a sufficient seed for runaway avalanche. Direct measurements of the runaway seed formation during thermal quench are not yet available. This remains to be a very challenging task motivated largely by the need to validate the hot-tail mechanism. Multiple complementary diagnostics now enable reconstruction of the runaway distribution function over the entire energy range. This distribution is found to decrease much faster than the one anticipated in a growing avalanche. Dissipation of the runaway electron current in the plateau regime is observed to be stronger than what would correspond to the estimated runaway sustainment threshold, although this comparison still needs to be refined to consider radial profiles of the relevant parameters and the presence of kinetic instabilities. Micro-instabilities receive an increased experimental attention lately as a plausible mechanism of runaway suppression. They can also provide a unique information about runaway evolution in phase space.

There are now important measurements of the runaway losses to the wall in the present devices, but the physics mechanism of these losses still needs to be ascertained in order to understand how the losses scale with the machine parameters and whether they result from the hypothesized intermittent leaks from the stochastic core through the good outer magnetic surfaces.

With ITER construction in progress, reliable means of runaway electron mitigation are yet to be developed. This is an essential part of the disruption mitigation strategy. Although theoretical and experimental understandings of runaway electrons behavior in current toka- 
maks have progressed significantly during the last decade, large uncertainties remain with respect to runaway control in ITER. A part of this challenge is to reconcile the physics and engineering constraints, as explained in Section XI of this review. 


\section{ACKNOWLEDGMENTS}

The authors are grateful for contributions and helpful comments from A. Fontanilla, P. Helander, V. Izzo, D. Kiramov, S. Konovalov, A. Loarte, E. Nardon, N. Marushchenko, H. Smith and J. Snipes.

This work was supported by ITER Contract No. ITER/CT/15/4300001178 and the U.S. Department of Energy Contract Nos. DEFG02-04ER54742 and DE-SC0016283.

ITER is the Nuclear Facility INB no. 174. The views and opinions expressed herein do not necessarily reflect those of the ITER Organization. This publication is provided for scientific purposes only. Its contents should not be considered as commitments from the ITER Organization as a nuclear operator in the frame of the licensing process.

[1] C. T. R. Wilson. The acceleration of $\beta$-particles in strong electric fields such as those of thunderclouds. Mathematical Proceedings of the Cambridge Philosophical Society, 22(04):534, mar 1925.

[2] H. Dreicer. Electron and ion runaway in a fully ionized gas. i. Phys. Rev., 115(2):238-249, Jul 1959.

[3] Y. A. Sokolov. Multiplication of accelerated electrons in a tokamak. JETP Letters, 29:218$221,1979$.

[4] R. Jayakumar, H.H. Fleischmann, and S.J. Zweben. Collisional avalanche exponentiation of runaway electrons in electrified plasmas. Physics Letters A, 172(6):447-451, Jan 1993.

[5] M.N. Rosenbluth and S.V. Putvinski. Theory for avalanche of runaway electrons in tokamaks. Nuclear Fusion, 37:1355, 1997.

[6] T.C. Hender, J.C Wesley, J. Bialek, A. Bondeson, A.H. Boozer, R.J. Buttery, A. Garofalo, T.P Goodman, R.S. Granetz, Y. Gribov, O. Gruber, M. Gryaznevich, G. Giruzzi, S. Günter, N. Hayashi, P. Helander, C.C. Hegna, D.F. Howell, D.A. Humphreys, G.T.A. Huysmans, A.W. Hyatt, A. Isayama, S.C. Jardin, Y. Kawano, A. Kellman, C. Kessel, H.R. Koslowski, R.J. La Haye, E. Lazzaro, Y.Q. Liu, V. Lukash, J. Manickam, S. Medvedev, V. Mertens, S.V. Mirnov, Y. Nakamura, G. Navratil, M. Okabayashi, T. Ozeki, R. Paccagnella, G. Pautasso, F. Porcelli, V.D. Pustovitov, V. Riccardo, M. Sato, O. Sauter, M.J. Schaffer, M. Shimada, 
P. Sonato, E.J. Strait, M. Sugihara, M. Takechi, A.D. Turnbull, E. Westerhof, D.G. Whyte, R. Yoshino, and H. Zohm. Chapter 3: Mhd stability, operational limits and disruptions. Nuclear Fusion, 47(6):S128, 2007.

[7] H. Knoepfel and D. A. Spong. Runaway electrons in toroidal discharges. Nuclear Fusion, 19(6):785-829, Jun 1979.

[8] V.V. Parail and O.P. Pogutse. "Runaway electrons in a tokamak." Reviews of plasma physics. Vol. II. Plenum Press; New York, NY (USA), 1986.

[9] MN Rosenbluth, PB Parks, D Post, S Putvinski, N Putvinskaya, and HA Scott. Runaway electrons and fast plasma shutdown. 16 Fusion Energy Conference Proceedings, Montreal, Canada, 7-11 October 1996, 2:979-986, 1996.

[10] M. Lehnen, K. Aleynikova, P.B. Aleynikov, D.J. Campbell, P. Drewelow, N.W. Eidietis, Yu. Gasparyan, R.S. Granetz, Y. Gribov, N. Hartmann, and et al. Disruptions in iter and strategies for their control and mitigation. Journal of Nuclear Materials, 463:39-48, Aug 2015 .

[11] E. M. Hollmann, P. B. Aleynikov, T Fulop, D. A. Humphreys, V. A. Izzo, M. Lehnen, V. E. Lukash, G. Papp, G. Pautasso, F. Saint-Laurent, and et al. Status of research toward the iter disruption mitigation system. Phys. Plasmas, 22(2):021802, Feb 2015.

[12] Allen H. Boozer. Runaway electrons and ITER. Nuclear Fusion, 57(5):056018, mar 2017.

[13] Allen H. Boozer. Pivotal issues on relativistic electrons in ITER. Nuclear Fusion, 58(3):036006, jan 2018.

[14] Robert G. Littlejohn. Hamiltonian formulation of guiding center motion. Physics of Fluids, 24(9):1730, 1981.

[15] H. K. Wimmel. Lagrangian formulation of a consistent relativistic guiding center theory. $Z$. Naturforsch, 38a:601-607, 1983.

[16] Meng Li, Boris N. Breizman, and Linjin Zheng. Canonical straight field line magnetic flux coordinates for tokamaks. Journal of Computational Physics, 326:334-341, dec 2016.

[17] S.S. Abdullaev, K. H. Finken, K Wongrach, and O Willi. Theoretical and experimental studies of runaway electrons in the textor tokamak. Technical report, Forschungszentrum Jülich GmbH Institute of Energy and Climate Research Plasma Physics IEK-4, 2016.

[18] Boris V Chirikov. A universal instability of many-dimensional oscillator systems. Physics Reports, 52(5):263-379, may 1979. 
[19] A. B. Rechester and M. N. Rosenbluth. Electron heat transport in a tokamak with destroyed magnetic surfaces. Physical Review Letters, 40(1):38-41, jan 1978.

[20] J. R. Myra, Peter J. Catto, H. E. Mynick, and R. E. Duvall. Quasilinear diffusion in stochastic magnetic fields: Reconciliation of drift-orbit modification calculations. Physics of Fluids B: Plasma Physics, 5(4):1160-1163, apr 1993.

[21] Konsta Särkimäki, Eero Hirvijoki, Joan Decker, Jari Varje, and Taina Kurki-Suonio. An advection-diffusion model for cross-field runaway electron transport in perturbed magnetic fields. Plasma Physics and Controlled Fusion, 58(12):125017, nov 2016.

[22] V.A Izzo. A numerical investigation of the effects of impurity penetration depth on disruption mitigation by massive high-pressure gas jet. Nuclear Fusion, 46(5):541-547, mar 2006.

[23] V. A. Izzo, D. G. Whyte, R. S. Granetz, P. B. Parks, E. M. Hollmann, L. L. Lao, and J. C. Wesley. Magnetohydrodynamic simulations of massive gas injection into alcator c-mod and DIII-d plasmas. Physics of Plasmas, 15(5):056109, may 2008.

[24] E Nardon, A Fil, M Hoelzl, and G Huijsmans and. Progress in understanding disruptions triggered by massive gas injection via $3 \mathrm{~d}$ non-linear MHD modelling with JOREK. Plasma Physics and Controlled Fusion, 59(1):014006, oct 2016.

[25] R. Yoshino and S. Tokuda. Runaway electrons in magnetic turbulence and runaway current termination in tokamak discharges. Nuclear Fusion, 40(7):1293, 2000.

[26] T. Hauff and F. Jenko. Runaway electron transport via tokamak microturbulence. Physics of Plasmas, 16(10):102308, oct 2009.

[27] G Papp, M Drevlak, T T Fulop, and G I Pokol. The effect of resonant magnetic perturbations on runaway electron transport in ITEr. Plasma Physics and Controlled Fusion, 54(12):125008, Nov 2012.

[28] V A Izzo, D A Humphreys, and M Kornbluth. Analysis of shot-to-shot variability in postdisruption runaway electron currents for diverted DIII-d discharges. Plasma Physics and Controlled Fusion, 54(9):095002, jul 2012.

[29] Sadrilla Abdullaev. Magnetic Stochasticity in Magnetically Confined Fusion Plasmas. Springer International Publishing, 2014.

[30] G. Papp, M. Drevlak, G. I. Pokol, and T. Fülöp. Energetic electron transport in the presence of magnetic perturbations in magnetically confined plasmas. Journal of Plasma Physics, 81(05), jul 2015. 
[31] C. Sommariva, E. Nardon, P. Beyer, M. Hoelzl, G.T.A. Huijsmans, and D. van Vugt and. Test particles dynamics in the JOREK 3d non-linear MHD code and application to electron transport in a disruption simulation. Nuclear Fusion, 58(1):016043, dec 2018.

[32] E. Hirvijoki, O. Asunta, T. Koskela, T. Kurki-Suonio, J. Miettunen, S. Sipilä, A. Snicker, and S. Äkäslompolo. ASCOT: Solving the kinetic equation of minority particle species in tokamak plasmas. Computer Physics Communications, 185(4):1310-1321, apr 2014.

[33] D. Del Castillo Negrete. Full orbit and backward monte carlo simulation of runaway electrons. PPPL TDS Workshop, 2017.

[34] P. Aleynikov, A.A. Ivanov, R.R. Khayrutdinov, S.V. Konovalov, V.E. Lukash, S.Yu. Medvedev, and S. Putvinski. Simulations of runaway electron transport under mhd perturbations in iter. In 37-th EPS conference on Plasma Physics, Dublin, Ireland, 2010.

[35] M. Lehnen, S. A. Bozhenkov, S. S. Abdullaev, and M. W. Jakubowski. Suppression of runaway electrons by resonant magnetic perturbations in TEXTOR disruptions. Physical Review Letters, 100(25), jun 2008.

[36] Allen H. Boozer and Alkesh Punjabi. Loss of relativistic electrons when magnetic surfaces are broken. Phys. Plasmas, 23:102513, 2016.

[37] L.-G. Eriksson and P. Helander. Simulation of runaway electrons during tokamak disruptions. Computer Physics Communications, 154(3):175 - 196, 2003.

[38] P. T. McCormick, D. G. Keiffer, and G. Parzen. Energy and angle distribution of electrons in bremsstrahlung. Physical Review, 103(1):29-31, jul 1956.

[39] AV Gurevich. On the theory of runaway electrons. Sov. Phys. JETP, 12(5):904-912, 1961.

[40] J.W. Connor and R.J. Hastie. Relativistic limitations on runaway electrons. Nuclear Fusion, 15(3):415-424, Jun 1975.

[41] G. Papp, M. Drevlak, T. Fulop, and P. Helander. Runaway electron drift orbits in magnetostatic perturbed fields. Nuclear Fusion, 51(4):043004, Mar 2011.

[42] Chr. Møller. Über den stoss zweier teilchen unter berücksichtigung der retardation der kräfte. Zeitschrift für Physik, 70(11-12):786-795, 1931.

[43] Chr. Møller. Zur theorie des durchgangs schneller elektronen durch materie. Annalen der Physik, 406(5):531-585, 1932.

[44] V. B. Berestetski, E. M. Lifshitz, and L. P. Pitaevskii. Quantum Electrodynamics (Course of Theoretical Physics, Vol. 4) (Volume 4). Pergamon, 1982. 
[45] Hans Bethe. Bremsformel für elektronen relativistischer geschwindigkeit. Zeitschrift für Physik, 76(5-6):293-299, 1932.

[46] A. A. Solodov and R. Betti. Stopping power and range of energetic electrons in dense plasmas of fast-ignition fusion targets. Physics of Plasmas, 15(4):042707, 2008.

[47] Martin J Berger, JS Coursey, MA Zucker, J Chang, et al. Stopping-power and range tables for electrons, protons, and helium ions. NIST Physics Laboratory Gaithersburg, MD, 1998.

[48] Felix Bloch. Bremsvermögen von atomen mit mehreren elektronen. Zeitschrift für Physik A Hadrons and Nuclei, 81(5):363-376, 1933.

[49] V. E. Zhogolev and S. V. Konovalov. Characteristics of interaction of energetic electrons with heavy impurity ions in a tokamak plasma. VANT series Nuclear Fusion, 37(4), 2014. in Russian.

[50] Hans Bethe. Zur theorie des durchgangs schneller korpuskularstrahlen durch materie. Annalen der Physik, 397(3):325-400, 1930.

[51] L. D. Landau and E.M. Lifshitz. Course of Theoretical Physics, Volume 8, Second Edition: Electrodynamics of Continuous Media. Pergamon, 1984.

[52] O. Embréus, A. Stahl, and T. Fülöp. On the relativistic large-angle electron collision operator for runaway avalanches in plasmas. Journal of Plasma Physics, 84(01), jan 2018.

[53] P. Aleynikov, K. Alynikova, B. Breizman, G. Huijsmans, S. Konovalov, S. Putvinski, and V. Zhogolev. Kinetic modeling of runaway electrons and their mitigation in iter. In Proceedings of the 25th IAEA Fusion Energy Conference, St. Petersburg, Russian Federation, pages 13-18, 2014.

[54] S.C Chiu, M.N Rosenbluth, R.W Harvey, and V.S Chan. Fokker-planck simulations mylb of knock-on electron runaway avalanche and bursts in tokamaks. Nuclear Fusion, 38(11):17111721, Nov 1998.

[55] N. F. Mott. The scattering of fast electrons by atomic nuclei. Proc. R. Soc. Lond. A, 124(794):425-442, 1929.

[56] VD Kirillov, BA Trubnikov, and SA Trushin. Role of impurities in anomalous plasma resistance. Fizika Plazmy, 1:218-237, 1975.

[57] Andris Gulans, Stefan Kontur, Christian Meisenbichler, Dmitrii Nabok, Pasquale Pavone, Santiago Rigamonti, Stephan Sagmeister, Ute Werner, and Claudia Draxl. Exciting: a full-potential all-electron package implementing density-functional theory and many-body 
perturbation theory. Journal of Physics: Condensed Matter, 26(36):363202, 2014.

[58] Linnea Hesslow, Ola Embréus, Adam Stahl, Tim C DuBois, Gergely Papp, Sarah L Newton, and Tünde Fülöp. Effect of partially screened nuclei on fast-electron dynamics. Physical review letters, 118(25):255001, 2017.

[59] David Mosher. Interactions of relativistic electron beams with high atomic-number plasmas. Physics of Fluids, 18(7):846, 1975.

[60] L. D. Landau and E.M. Lifshitz. Classical Theory of Fields. Pergamon, 1971.

[61] Julian Schwinger. On the classical radiation of accelerated electrons. Physical Review, 75(12):1912-1925, jun 1949.

[62] V. V. Zharkova, K. Arzner, A. O. Benz, P. Browning, C. Dauphin, A. G. Emslie, L. Fletcher, E. P. Kontar, G. Mann, M. Onofri, V. Petrosian, R. Turkmani, N. Vilmer, and L. Vlahos. Recent advances in understanding particle acceleration processes in solar flares. Space Science Reviews, 159(1-4):357-420, jul 2011.

[63] I. Fernandez-Gomez, J. R. Martin-Solis, and R. Sanchez. Determination of the parametric region in which runaway electron energy losses are dominated by bremsstrahlung radiation in tokamaks. Phys. Plasmas, 14(7):072503, 2007.

[64] V.L. Ginzburg and D. Haar. Theoretical Physics and Astrophysics. International Series on Nuclear Energy. Elsevier Science, 2013.

[65] IM Pankratov. Analysis of the synchrotron radiation spectra of runaway electrons. PLASMA PHYSICS REPORTS C/C OF FIZIKA PLAZMY, 25:145-148, 1999.

[66] Ya M Sobolev. Polarization of synchrotron radiation from relativistic electrons moving within toroidal magnetic fields. Problems of Atomic Science and Technology, 4(86):108, 2013.

[67] F. Andersson, P. Helander, and L.-G. Eriksson. Damping of relativistic electron beams by synchrotron radiation. Phys. Plasmas, 8(12):5221, 2001.

[68] Fredrik Andersson. Runaway Electrons in Tokamak Plasmas. PhD thesis, Chalmers University of Technology, 2003.

[69] J Decker, E Hirvijoki, O Embreus, Y Peysson, A Stahl, I Pusztai, and T Fülöp. Numerical characterization of bump formation in the runaway electron tail. Plasma Physics and Controlled Fusion, 58(2):025016, jan 2016.

[70] Allen H. Boozer. Theory of runaway electrons in ITER: Equations, important parameters, and implications for mitigation. Physics of Plasmas, 22(3):032504, mar 2015. 
[71] H. Bethe and W. Heitler. On the stopping of fast particles and on the creation of positive electrons. Proceedings of the Royal Society A: Mathematical, Physical and Engineering Sciences, 146(856):83-112, aug 1934.

[72] Fritz Sauter. Über die bremsstrahlung schneller elektronen. Annalen der Physik, 412(4):404$412,1934$.

[73] Giulio Racah. Sopra l'irradiazione nell'urto di particelle veloci. Il Nuovo Cimento (19241942), 11(7):461-476, 1934.

[74] D. C. Pace, C. M. Cooper, D. Taussig, N. W. Eidietis, E. M. Hollmann, V. Riso, M. A. Van Zeeland, and M. Watkins. Gamma ray imager on the DIII-d tokamak. Review of Scientific Instruments, 87(4):043507, apr 2016.

[75] CM Cooper, DC Pace, C Paz-Soldan, N Commaux, NW Eidietis, EM Hollmann, and D Shiraki. Applying the new gamma ray imager diagnostic to measurements of runaway electron bremsstrahlung radiation in the diii-d tokamak. Review of Scientific Instruments, 87(11):11E602, 2016.

[76] Carlos Paz-Soldan, Christopher M Cooper, Pavel Aleynikov, David C Pace, Nicholas W Eidietis, Dylan P Brennan, RS Granetz, Eric M Hollmann, C Liu, Andrey Lvovskiy, et al. Spatiotemporal evolution of runaway electron momentum distributions in tokamaks. Physical review letters, 118(25):255002, 2017.

[77] Felix Bloch and Arnold Nordsieck. Note on the radiation field of the electron. Physical Review, 52(2):54, 1937.

[78] O Embréus, A Stahl, and T Fülöp. Effect of bremsstrahlung radiation emission on fast electrons in plasmas. New Journal of Physics, 18(9):093023, sep 2016.

[79] L. Landau and E. Lifshitz. On the production of electrons and positrons by a collision of two particles. Phys. Z. Sowjet., 6(244), 1934.

[80] D. A. Gryaznykh. Cross section for the production of electron-positron pairs by electrons in the field of a nucleus. Physics of Atomic Nuclei, 61(3):394-399, 1998.

[81] O. Embréus, L. Hesslow, M. Hoppe, G. Papp, K. Richards, and T. Fülöp. Dynamics of positrons during relativistic electron runaway. Journal of Plasma Physics, 84(5), oct 2018.

[82] P. Helander and D. J. Ward. Positron creation and annihilation in tokamak plasmas with runaway electrons. Physical Review Letters, 90(13), Apr 2003.

[83] Tünde Fülöp and Gergely Papp. Runaway positrons in fusion plasmas. Physical review 
letters, 108(22):225003, 2012.

[84] P. C. de Vries, M. Baruzzo, G. M. D. Hogeweij, S. Jachmich, E. Joffrin, P. J. Lomas, G. F. Matthews, A. Murari, I. Nunes, T. Pütterich, C. Reux, and J. Vega and. The influence of an ITER-like wall on disruptions at JET. Physics of Plasmas, 21(5):056101, may 2014.

[85] E.M. Hollmann, N. Commaux, R.A. Moyer, P.B. Parks, M.E. Austin, I. Bykov, C. Cooper, N.W. Eidietis, M. O'Mullane, C. Paz-Soldan, D.L. Rudakov, and D. Shiraki. Use of ar pellet ablation rate to estimate initial runaway electron seed population in DIII-d rapid shutdown experiments. Nuclear Fusion, 57(1):016008, oct 2017.

[86] P.C. de Vries, M.F. Johnson, B. Alper, P. Buratti, T.C. Hender, H.R. Koslowski, and V. Riccardo and. Survey of disruption causes at JET. Nuclear Fusion, 51(5):053018, apr 2011.

[87] D.J Ward and J.A Wesson. Impurity influx model of fast tokamak disruptions. Nuclear Fusion, 32(7):1117-1123, jul 1992.

[88] S. I. Braginskii. Review of Plasma Physics, Vol. 1. Consultants Bureau, New York, 1965.

[89] Pavel Aleynikov and Boris N. Breizman. Generation of runaway electrons during the thermal quench in tokamaks. Nuclear Fusion, 57(4):046009, feb 2017.

[90] HP Summers. Atomic data and analysis structure: user manual. Technical report, Joint European Torus, 1994.

[91] P. Aleynikov, B.N. Breizman, P. Helander, and Yu. Turkin. Plasma ion heating by cryogenic pellet injection. Journal of Plasma Physics, 2018.

[92] R. W. Harvey, V. S. Chan, S. C. Chiu, T. E. Evans, M. N. Rosenbluth, and D. G. Whyte. Runaway electron production in diii-d killer pellet experiments, calculated with the cql3d/kprad model. Phys. Plasmas, 7(11):4590, 2000.

[93] P. Helander, H. Smith, T Fulop, and L.-G. Eriksson. Electron kinetics in a cooling plasma. Phys. Plasmas, 11(12):5704, 2004.

[94] H. Smith, P. Helander, L.-G. Eriksson, and T Fulop. Runaway electron generation in a cooling plasma. Phys. Plasmas, 12(12):122505, 2005.

[95] H. M. Smith and E. Verwichte. Hot tail runaway electron generation in tokamak disruptions. Phys. Plasmas, 15(7):072502, 2008.

[96] T Feher, H M Smith, T Fulop, and K Gal. Simulation of runaway electron generation during plasma shutdown by impurity injection in iter. Plasma Physics and Controlled Fusion, 53(3):035014, Feb 2011. 
[97] H. Dreicer. Electron and ion runaway in a fully ionized gas. ii. Phys. Rev., 117(2):329-342, Jan 1960.

[98] AN Lebedev. Contribution to the theory of runaway electrons. Sov. Phys. JETP, 21:931-933, 1965.

[99] Martin D. Kruskal and Ira B. Bernstein. Runaway electrons in an ideal lorentz plasma. Physics of Fluids, 7(3):407, 1964.

[100] Ronald H. Cohen. Runaway electrons in an impure plasma. Physics of Fluids, 19(2):239, 1976.

[101] J.R. Martin-Solis, A. Loarte, and M. Lehnen. Formation and termination of runaway beams in ITER disruptions. Nuclear Fusion, 57(6):066025, apr 2017.

[102] E Nilsson, J Decker, Y Peysson, R S Granetz, F Saint-Laurent, and M Vlainic. Kinetic modelling of runaway electron avalanches in tokamak plasmas. Plasma Physics and Controlled Fusion, 57(9):095006, jul 2015.

[103] R.E. Ismailov, P. Aleynikov, and S.V. Konovalov. Dreicer mechanism of runaway electron generation in presence of high-z impurities. In 43rd EPS conference on Plasma Physics, Leuven, Belgium, 2016.

[104] O. Klein and Y. Nishina. Über die streuung von strahlung durch freie elektronen nach der neuen relativistischen quantendynamik von dirac. Zeitschrift für Physik, 52(11-12):853-868, nov 1929.

[105] Markus J. Aschwanden. Physics of the Solar Corona. Springer Berlin Heidelberg, 2006.

[106] Masaaki Yamada, Jongsoo Yoo, and Clayton E. Myers. Understanding the dynamics and energetics of magnetic reconnection in a laboratory plasma: Review of recent progress on selected fronts. Physics of Plasmas, 23(5):055402, may 2016.

[107] P. V. Savrukhin. Generation of suprathermal electrons during magnetic reconnection at the sawtooth crash and disruption instability in the t-10 tokamak. Physical Review Letters, 86(14):3036-3039, apr 2001.

[108] N. Chu, Y. Sun, S. Gu, H.H. Wang, Y.J. Hu, T.H. Shi, D.L. Chen, X. Gu, K.Y. He, M. Jia, S.Y. Lin, H.Q. Liu, J.P. Qian, J. Ren, B. Shen, A. Ti, S.X. Wang, J.L. Xie, M. Xu, M.M. Xue, N. Yan, Q. Zang, L. Zeng, J.Z. Zhang, T. Zhang, Y. Zhang, G.Q. Zhong, C. Zhou, and R.J. Zhou. Observation of toroidal alfvén eigenmode excited by energetic electrons induced by static magnetic perturbations in the EAST tokamak. Nuclear Fusion, 58(10):104004, aug 
2018.

[109] P Helander, L-G Eriksson, and F Andersson. Runaway acceleration during magnetic reconnection in tokamaks. Plasma Physics and Controlled Fusion, 44(12B):B247-B262, Nov 2002.

[110] K G McClements, J O Allen, S C Chapman, R O Dendy, S W A Irvine, O Marshall, D Robb, M Turnyanskiy, and R G L Vann. Particle acceleration during merging-compression plasma start-up in the mega amp spherical tokamak. Plasma Physics and Controlled Fusion, 60(2):025013, dec 2017.

[111] B.A. Trubnikov. Review of Plasma Physics, Vol. 1. Consultants Bureau, New York, 1965.

[112] J.R Martín-Solís, B Esposito, R Sánchez, G Granucci, and L Panaccione. Comparison of runaway dynamics in LH and ECRH heated discharges in the frascati tokamak upgrade. Nuclear Fusion, 45(12):1524-1533, nov 2005.

[113] J. R. Martin-Solis, A. Loarte, and M. Lehnen. On the avalanche generation of runaway electrons during tokamak disruptions. Phys. Plasmas, 22(8):082503, Aug 2015.

[114] L.-G. Eriksson, P. Helander, F. Andersson, D. Anderson, and M. Lisak. Current dynamics during disruptions in large tokamaks. Physical Review Letters, 92(20), May 2004.

[115] P. Aleynikov. Assessment of runaway seed generation and its suppression by disruption mitigation. CONTRACT NUMBER IO/CT/4300001355, Final Report, 2017.

[116] M. Bakhtiari, G. J. Kramer, M. Takechi, H. Tamai, Y. Miura, Y. Kusama, and Y. Kamada. Role of bremsstrahlung radiation in limiting the energy of runaway electrons in tokamaks. Physical Review Letters, 94(21), Jun 2005.

[117] M. Bakhtiari, G. J. Kramer, and D. G. Whyte. Momentum-space study of the effect of bremsstrahlung radiation on the energy of runaway electrons in tokamaks. Phys. Plasmas, 12(10):102503, 2005.

[118] K.O. Aleynikova, P.B. Aleynikov, Konovalov S.V., Teplukhina A.A., and Zhogolev V.E. Interaction of runaway electrons with high-z impurities. In 40-th EPS conference on Plasma Physics, Espoo, Finland, O5.103, 2013.

[119] J. R. Martin-Solis, J. D. Alvarez, R. Sanchez, and B. Esposito. Momentum-space structure of relativistic runaway electrons. Phys. Plasmas, 5(6):2370, 1998.

[120] Pavel Aleynikov and Boris N. Breizman. Theory of two threshold fields for relativistic runaway electrons. Physical Review Letters, 114(15), Apr 2015. 
[121] E. Hirvijoki, I. Pusztai, J. Decker, O. Embreus, A. Stahl, and T. Fulop. Radiation reaction induced non-monotonic features in runaway electron distributions. J. Plasma Phys., 81(05), jul 2015.

[122] Zehua Guo, Xian-Zhu Tang, and Christopher J. McDevitt. Models of primary runaway electron distribution in the runaway vortex regime. Physics of Plasmas, 24(11):112508, nov 2017.

[123] Adrian K. Fontanilla and Boris N. Breizman. Lifetime and universal distribution of seed runaway electrons. Physics of Plasmas, 24(11):112509, nov 2017.

[124] Chang Liu, Dylan P. Brennan, Amitava Bhattacharjee, and Allen H. Boozer. Adjoint fokkerplanck equation and runaway electron dynamics. Physics of Plasmas, 23(1):010702, jan 2016.

[125] Pavel Aleynikov and Boris Breizman. Relativistic runaway electrons in a near-threshold electric field. In 42-nd EPS conference on Plasma Physics, Lisbon, Portugal, 2015.

[126] Zehua Guo, Christopher J McDevitt, and Xian-Zhu Tang. Phase-space dynamics of runaway electrons in magnetic fields. Plasma Physics and Controlled Fusion, 59(4):044003, feb 2017.

[127] B.N. Breizman and P.B. Aleynikov. Kinetics of relativistic runaway electrons. Nuclear Fusion, 57(12):125002, oct 2017 .

[128] Christopher J McDevitt, Zehua Guo, and Xian-Zhu Tang. Relation of the runaway avalanche threshold to momentum space topology. Plasma Physics and Controlled Fusion, 60(2):024004, jan 2018.

[129] L Hesslow, O Embréus, G J Wilkie, G Papp, and T Fülöp. Effect of partially ionized impurities and radiation on the effective critical electric field for runaway generation. Plasma Physics and Controlled Fusion, 60(7):074010, jun 2018.

[130] Boris N. Breizman. Marginal stability model for the decay of runaway electron current. Nuclear Fusion, 54(7):072002, Apr 2014.

[131] H. Smith, P. Helander, L.-G. Eriksson, D. Anderson, M. Lisak, and F. Andersson. Runaway electrons and the evolution of the plasma current in tokamak disruptions. Phys. Plasmas, 13(10):102502, 2006.

[132] S. Konovalov, P. Aleynikov, K. Aleynikova, Yu. Gribov, R. Ismailov, A. Ivanov, R. Khayrutdinov, D. Kiramov, M. Lehnen, V. Leonov, V. Lukash, S. Medvedev, and V. Zhogolev. Assessment of the runaway electron energy dissipation in iter. in Proceedings of the 26th IAEA Fusion Energy Conference, Kyoto, Japan, TH/7-1, 2016. IAEA FEC. 
[133] K. Aleynikova, G. T. A. Huijsmans, and P. Aleynikov. Linear mhd stability analysis of post-disruption plasmas in iter. Plasma Physics Reports, 42(5):486-494, may 2016.

[134] P. Helander, D. Grasso, R. J. Hastie, and A. Perona. Resistive stability of a plasma with runaway electrons. Phys. Plasmas, 14(12):122102, 2007.

[135] S Putvinski, P Barabaschi, N Fujisawa, N Putvinskaya, M N Rosenbluth, and J Wesley. Halo current, runaway electrons and disruption mitigation in ITER. Plasma Physics and Controlled Fusion, 39(12B):B157-B171, dec 1997.

[136] A. Loarte, V. Riccardo, J. R. Martin-Solis, J. Paley, A. Huber, and M. Lehnen. Magnetic energy flows during the current quench and termination of disruptions with runaway current plateau formation in jet and implications for ITEr. Nuclear Fusion, 51(7):073004, May 2011.

[137] J. Riemann, H. M. Smith, and P. Helander. Energetics of runaway electrons during tokamak disruptions. Phys. Plasmas, 19(1):012507, 2012.

[138] S. Konovalov, P. Aleynikov, K. Aleynikova, Yu. Gribov, G.T.A. Huijsmans, A. Ivanov, R. Khayrutdinov, M. Lehnen, V. Leonov, V. Lukash, S. Medvedev, and V. Zhogolev. Integrated modelling of iter disruption mitigation. in Proceedings of the 25th IAEA Fusion Energy Conference, St. Petersburg, Russian Federation, TH/P3-38., 2014.

[139] P. L. Taylor, A. G. Kellman, T. E. Evans, D. S. Gray, D. A. Humphreys, A. W. Hyatt, T. C. Jernigan, R. L. Lee, J. A. Leuer, S. C. Luckhardt, P. B. Parks, M. J. Schaffer, D. G. Whyte, and J. Zhang. Disruption mitigation studies in DIII-d. Physics of Plasmas, 6(5):1872-1879, may 1999.

[140] M. Lehnen, G. Arnoux, S. Brezinsek, J. Flanagan, S.N. Gerasimov, N. Hartmann, T.C. Hender, A. Huber, S. Jachmich, V. Kiptily, and et al. Impact and mitigation of disruptions with the iter-like wall in jet. Nuclear Fusion, 53(9):093007, Aug 2013.

[141] R.R. Khayrutdinov and V.E. Lukash. Studies of plasma equilibrium and transport in a tokamak fusion device with the inverse-variable technique. Journal of Computational Physics, 109(2):193-201, dec 1993.

[142] A. Fil, E. Nardon, M. Hoelzl, G. T. A Huijsmans, F. Orain, M. Becoulet, P. Beyer, G. DifPradalier, R. Guirlet, H. R. Koslowski, M. Lehnen, J. Morales, S. Pamela, C. Passeron, C. Reux, and F. Saint-Laurent and. Three-dimensional non-linear magnetohydrodynamic modeling of massive gas injection triggered disruptions in JET. Physics of Plasmas, 22(6):062509, jun 2015. 
[143] G T A Huysmans, S Pamela, E van der Plas, and P Ramet. Non-linear MHD simulations of edge localized modes (ELMs). Plasma Physics and Controlled Fusion, 51(12):124012, nov 2009 .

[144] D. I. Kiramov and B. N. Breizman. Model of vertical plasma motion during the current quench. Physics of Plasmas, 24(10):100702, oct 2017.

[145] D. Pfefferlé and A. Bhattacharjee. Algebraic motion of vertically displacing plasmas. Physics of Plasmas, 25(2):022516, feb 2018.

[146] ITER Physics Expert Group on Disrup MHD and ITER Physics Basis Editors. Chapter 3: MHD stability, operational limits and disruptions. Nuclear Fusion, 39(12):2251-2389, dec 1999.

[147] Jeffrey P. Freidberg. Plasma Physics and Fusion Energy. Cambridge University Press, 2007.

[148] D.I. Kiramov. private communication. private communication, 2018.

[149] S. Konovalov, P. Aleynikov, K. Aleynikova, Yu. Gribov, R. Ismailov, A. Ivanov, R. Khayrutdinov, D. Kiramov, M. Lehnen, V. Leonov, V. Lukash, S. Medvedev, and V. Zhogolev. Assessment of the runaway electron energy dissipation in iter. 30th MHD ITPA, Barcelona, Spain, TH/7-1, 2017.

[150] VV Alikaev, KA Razumova, and Yu A Sokolov. Runaway-electron instability in the tm-3 tokamak. Sov. J. Plasma Phys.(Engl. Transl.);(United States), 1(4), 1975.

[151] V.S. Vlasenkov, V.M. Leonov, V.G. Merezhkin, and V.S. Mukhovatov. The runaway electron discharge regime in the tokamak-6 device. Nuclear Fusion, 13(4):509-516, Aug 1973.

[152] Equipe TFR. High-current discharges in the tfr device. In 5. international conference on plasma physics and controlled nuclear fusion research; Tokyo, Japan; IAEA-CN-33/A6-2, 1974 .

[153] E.D. Fredrickson, M.G. Bell, G. Taylor, and S.S. Medley. Control of disruption-generated runaway plasmas in tftr. Nuclear Fusion, 55(1):013006, Dec 2014.

[154] D. A. Spong, W. W. Heidbrink, C. Paz-Soldan, X. D. Du, K. E. Thome, M. A. Van Zeeland, C. Collins, A. Lvovskiy, R. A. Moyer, M. E. Austin, D. P. Brennan, C. Liu, E. F. Jaeger, and C. Lau. First direct observation of runaway-electron-driven whistler waves in tokamaks. Physical Review Letters, 120(15), apr 2018.

[155] V.V. Parail and O.P. Pogutse. The kinetic theory of runaway electron beam instability in a tokamak. Nuclear Fusion, 18(3):303-314, Mar 1978. 
[156] T Fulop, G. Pokol, P. Helander, and M. Lisak. Destabilization of magnetosonic-whistler waves by a relativistic runaway beam. Phys. Plasmas, 13(6):062506, 2006.

[157] G Pokol, T Fulop, and M Lisak. Quasi-linear analysis of whistler waves driven by relativistic runaway beams in tokamaks. Plasma Physics and Controlled Fusion, 50(4):045003, Feb 2008.

[158] T. Fulop, H. M. Smith, and G. Pokol. Magnetic field threshold for runaway generation in tokamak disruptions. Phys. Plasmas, 16(2):022502, 2009.

[159] Pavel Aleynikov and Boris Breizman. Stability analysis of runaway-driven waves in a tokamak. Nuclear Fusion, 55(4):043014, Mar 2015.

[160] Marco Brambilla. The effects of coulomb collisions on the propagation of cold-plasma waves. Physics of Plasmas, 2(4):1094-1099, apr 1995.

[161] Chang Liu, Lei Shi, Eero Hirvijoki, Dylan P. Brennan, Amitava Bhattacharjee, Carlos PazSoldan, and Max E. Austin. The effects of kinetic instabilities on the electron cyclotron emission from runaway electrons. Nuclear Fusion, 58(9):096030, jul 2018.

[162] A. Smirnov, R. Harvey, and K. Kupfer. A general ray tracing code genray. Bull Amer. Phys. Soc, 39(7):1626, 1994.

[163] A. P. Smirnov and R.W. Harvey. The GENRAY Ray Tracing Code, 2003.

[164] Chang Liu, Eero Hirvijoki, Guo-Yong Fu, Dylan P. Brennan, Amitava Bhattacharjee, and Carlos Paz-Soldan. Role of kinetic instability in runaway-electron avalanches and elevated critical electric fields. Physical Review Letters, 120(26), jun 2018.

[165] R. W. Harvey and M. G. McCoy. The cql3d code. In Proc. IAEA TCM on Advances in Sim. and Modeling of Thermonuclear Plasmas, Montreal, pages 489-526, 1992.

[166] Y. Peysson and J. Decker. Calculation of rf current drive in tokamaks. AIP Conference Proceedings, 1069(176), 2008.

[167] J. R. Martin-Solis, B. Esposito, R. R. Sanchez, and J. D. Alvarez. Energy limits on runaway electrons in tokamak plasmas. Phys. Plasmas, 6(1):238, 1999.

[168] AE Costley, RJ Hastie, JWM Paul, and J Chamberlain. Electron cyclotron emission from a tokamak plasma: experiment and theory. Physical Review Letters, 33(13):758, 1974.

[169] K.H. Finken, J.G. Watkins, D. Rusbüldt, W.J. Corbett, K.H. Dippel, D.M. Goebel, and R.A. Moyer. Observation of infrared synchrotron radiation from tokamak runaway electrons in textor. Nuclear Fusion, 30(5):859-870, May 1990.

[170] B. Esposito, J. R. Martín-Solís, F. M. Poli, J. A. Mier, R. Sánchez, and L. Panaccione. 
Dynamics of high energy runaway electrons in the frascati tokamak upgrade. Phys. Plasmas, 10(6):2350-2360, 2003.

[171] P Blanchard, S Alberti, S Coda, H Weisen, P Nikkola, and I Klimanov. High field side measurements of non-thermal electron cyclotron emission on tcv plasmas with ech and eccd. Plasma physics and controlled fusion, 44(10):2231, 2002.

[172] E.M. Hollmann, M.E. Austin, J.A. Boedo, N.H. Brooks, N. Commaux, N.W. Eidietis, D.A. Humphreys, V.A. Izzo, A.N. James, T.C. Jernigan, and et al. Control and dissipation of runaway electron beams created during rapid shutdown experiments in diii-d. Nuclear Fusion, 53(8):083004, Jul 2013.

[173] Massimo Nocente, Marco Tardocchi, Robin Barnsley, Luciano Bertalot, Benoît Brichard, Gabriele Croci, Giorgio Brolatti, L Di Pace, A Fernandes, Luca Giacomelli, et al. Conceptual design of the radial gamma ray spectrometers system for $\alpha$ particle and runaway electron measurements at iter. Nuclear Fusion, 57(7):076016, 2017.

[174] A.N. James, M.E. Austin, N. Commaux, N.W. Eidietis, T.E. Evans, E.M. Hollmann, D.A. Humphreys, A.W. Hyatt, V.A. Izzo, T.C. Jernigan, and et al. Measurements of hard x-ray emission from runaway electrons in diii-d. Nuclear Fusion, 52(1):013007, Dec 2011.

[175] PV Savrukhin. Measurements of the nonthermal x-ray emission in the t-10 tokamak using cdte detectors. Review of scientific instruments, 73(12):4243-4245, 2002.

[176] A.E. Shevelev, E.M. Khilkevitch, V.G. Kiptily, I.N. Chugunov, D.B. Gin, D.N. Doinikov, V.O. Naidenov, A.E. Litvinov, and I.A. Polunovskii. Reconstruction of distribution functions of fast ions and runaway electrons in fusion plasmas using gamma-ray spectrometry with applications to ITEr. Nuclear Fusion, 53(12):123004, Nov 2013.

[177] B. Kurzan, K. H. Steuer, and G. Fussmann. Dynamics of runaway electrons in the magnetic field of a tokamak. Physical Review Letters, 75(25):4626-4629, Dec 1995.

[178] Stephen M Seltzer and Martin J Berger. Bremsstrahlung spectra from electron interactions with screened atomic nuclei and orbital electrons. Nuclear Instruments and Methods in Physics Research Section B: Beam Interactions with Materials and Atoms, 12(1):95-134, 1985.

[179] Yung-Su Tsai. Pair production and bremsstrahlung of charged leptons. Reviews of Modern Physics, 46(4):815, 1974.

[180] EM Hollmann, L Chousal, RK Fisher, R Hernandez, GL Jackson, MJ Lanctot, SV Pidcoe, 
J Shankara, and DA Taussig. Soft x-ray array system with variable filters for the diii-d tokamak. Review of Scientific Instruments, 82(11):113507, 2011.

[181] William R Hendee and E Russell Ritenour. Medical imaging physics. Year Book Medical Pub, 1992.

[182] ZY Chen, BN Wan, SY Lin, YJ Shi, LQ Hu, XZ Gong, H Lin, and M Asif. Measurement of the runaway electrons in the ht-7 tokamak. Review of scientific instruments, 77(1):013502, 2006 .

[183] C Rasouli, D Iraji, AH Farahbod, K Akhtari, H Rasouli, H Modarresi, and M Lamehi. Runaway electron energy measurement using hard x-ray spectroscopy in "damavand" tokamak. Review of Scientific Instruments, 80(1):013503, 2009.

[184] Milos Vlainic, Jan Mlynar, Jordan Cavalier, Vladimir Weinzettl, Richard Paprok, Martin Imrisek, Ondrej Ficker, Mykyta Varavin, Petr Vondracek, J-M Noterdaeme, et al. Postdisruptive runaway electron beams in the compass tokamak. Journal of Plasma Physics, $81(5), 2015$.

[185] AE Shevelev, EM Khilkevitch, SI Lashkul, VV Rozhdestvensky, AB Altukhov, IN Chugunov, DN Doinikov, LA Esipov, DB Gin, MV Iliasova, et al. High performance gamma-ray spectrometer for runaway electron studies on the ft-2 tokamak. Nuclear Instruments and Methods in Physics Research Section A: Accelerators, Spectrometers, Detectors and Associated Equipment, 830:102-108, 2016.

[186] Equipe TFR. Tokamak plasma diagnostics. Nuclear Fusion, 18(5):647-731, 1978.

[187] A. Shevelev, V. Kiptily, I. Chugunov, E. Khilkevitch, D. Gin, D. Doinikov, V. Naidenov, V. Plyusnin, and EFDA-JET contributors. Study of runaway electrons with hard x-ray spectrometry of tokamak plasmas. AIP Publishing LLC, 2014.

[188] K. S. Cheng and J. L. Zhang. General radiation formulae for a relativistic charged particle moving in curved magnetic field lines: The synchrocurvature radiation mechanism. Astrophys. J., 463:271, 1996.

[189] A. Stahl, M. Landreman, G. Papp, E. Hollmann, and T Fulop. Synchrotron radiation from a runaway electron distribution in tokamaks. Phys. Plasmas, 20(9):093302, 2013.

[190] R A Tinguely, R S Granetz, M Hoppe, and O Embréus. Spatiotemporal evolution of runaway electrons from synchrotron images in alcator c-mod. Plasma Physics and Controlled Fusion, 60(12):124001, oct 2018 . 
[191] JH Yu, EM Hollmann, N Commaux, NW Eidietis, DA Humphreys, AN James, Thomas C Jernigan, and RA Moyer. Visible imaging and spectroscopy of disruption runaway electrons in diii-d. Phys. Plasmas, 20(4):042113, 2013.

[192] Mathias Hoppe, Ola Embreus, Carlos Paz-Soldan, Richard A Moyer, and Tunde Fulop. Interpretation of runaway electron synchrotron and bremsstrahlung images. Nuclear Fusion, 2018.

[193] ME Austin and J Lohr. Electron cyclotron emission radiometer upgrade on the diii-d tokamak. Review of scientific instruments, 74(3):1457-1459, 2003.

[194] WHM Clark. The precision of electron cyclotron emission measurements from dite tokamak. Plasma physics, 25(12):1501, 1983.

[195] C.M. Celata and D.A. Boyd. Cyclotron radiation as a diagnostic tool for tokamak plasmas. Nuclear Fusion, 17(4):735-759, aug 1977.

[196] C. Paz-Soldan, R. J. La Haye, D. Shiraki, R. J. Buttery, N. W. Eidietis, E. M. Hollmann, R. A. Moyer, J. E. Boom, and I. T. Chapman and. The non-thermal origin of the tokamak low-density stability limit. Nuclear Fusion, 56:056010, 2016.

[197] Kosuke Kato and Ian H Hutchinson. Alcator c vertical viewing electron cyclotron emission diagnostic. Review of Scientific Instruments, 57(8):1959-1961, 1986.

[198] W. W. Eshetua, L. Porte, A. Fasoli, O. Sauter, S. Coda, and T. P. Goodman and. Vertical electron cyclotron emission diagnostic for tcv plasmas. EPJ Web of Conferences, 32:03011, 2012.

[199] S Preische, PC Efthimion, and SM Kaye. Oblique electron cyclotron emission for electron distribution studies. Review of scientific instruments, 68(1):409-414, 1997.

[200] RL Freeman and Eric M Jones. Atomic collision processes in plasma physics experiments. Technical report, UKAEA Research Group, 1974.

[201] Timur Kudyakov, KH Finken, MW Jakubowski, M Lehnen, Y Xu, Bernd Schweer, T Toncian, G Van Wassenhove, O Willi, TEXTOR team, et al. Spatially and temporally resolved measurements of runaway electrons in the textor tokamak. Nuclear fusion, 48(12):122002, 2008 .

[202] Wook Jae Yoo, Sang Hun Shin, Dayeong Jeon, Seunghan Hong, Seon Geun Kim, Hyeok In Sim, Kyoung Won Jang, Seunghyun Cho, and Bongsoo Lee. Simultaneous measurements of pure scintillation and cerenkov signals in an integrated fiber-optic dosimeter for electron 
beam therapy dosimetry. Optics Express, 21(23):27770, nov 2013.

[203] Lech Jakubowski, Marek J Sadowski, Jaroslaw Zebrowski, Karol Malinowski, Marek Rabinski, Marcin J Jakubowski, and Robert Mirowski. Cherenkov-type diagnostics of fast electrons within tokamak plasmas. Physica Scripta, 2014(T161):014011, 2014.

[204] J Zebrowski, L Jakubowski, M Rabinski, M J Sadowski, M J Jakubowski, R Kwiatkowski, K Malinowski, R Mirowski, J Mlynar, O Ficker, V Weinzettl, F Causa, and and. Studies of runaway electrons via cherenkov effect in tokamaks. Journal of Physics: Conference Series, 959:012002, jan 2018.

[205] M. Forster, K. H. Finken, M. Lehnen, O. Willi, Y. Xu, and the TEXTOR Team. Measurements of the runaway electron energy during disruptions in the tokamak textor. Phys. Plasmas, 19(5):052506, 2012.

[206] M. Lehnen, S.S. Abdullaev, G. Arnoux, S.A. Bozhenkov, M.W. Jakubowski, R. Jaspers, V.V. Plyusnin, V. Riccardo, and U. Samm. Runaway generation during disruptions in jet and textor. Journal of Nuclear Materials, 390-391:740-746, Jun 2009.

[207] Eric M Hollmann, Nicolas Commaux, NW Eidietis, Charles J Lasnier, Dimitry L Rudakov, Daisuke Shiraki, Christopher Cooper, JR Martin-Solis, PB Parks, and Carlos Paz-Soldan. Study of z scaling of runaway electron plateau final loss energy deposition into wall of diii-d. Phys. Plasmas, 24(6):062505, 2017.

[208] E Delchambre, G Counsell, and A Kirk. Effect of micrometric hot spots on surface temperature measurement and flux calculation in the middle and long infrared. Plasma Physics and Controlled Fusion, 51(5):055012, 2009.

[209] C Zhang, E Gauthier, C Pocheau, C Balorin, JY Pascal, M Jouve, MH Aumeunier, X Courtois, Th Loarer, and M Houry. Surface temperature measurement of the plasma facing components with the multi-spectral infrared thermography diagnostics in tokamaks. Infrared Physics $\&$ Technology, 81:215-222, 2017.

[210] E. M. Hollmann, P. B. Parks, N. Commaux, N. W. Eidietis, R. A. Moyer, D. Shiraki, M. E. Austin, C. J. Lasnier, C. Paz-Soldan, and D. L. Rudakov. Measurement of runaway electron energy distribution function during high-z gas injection into runaway electron plateaus in diii-da). Phys. Plasmas, 22(5):056108, May 2015.

[211] M.-H. Aumeunier, M. Kočan, R. Reichle, and E. Gauthier. Impact of reflections on the divertor and first wall temperature measurements from the ITER infrared imaging system. 
Nuclear Materials and Energy, 12:1265-1269, aug 2017.

[212] Takaichi Kawamura, Haruo Obayashi, and Akira Miyahara. On the generation of runaway electrons and their impact to plasma facing components. Fusion Engineering and Design, 9:39-44, 1989.

[213] F. Causa, P. Buratti, B. Esposito, G. Pucella, E. Giovannozzi, L. Jakubowski, K. Malinowski, M. Rabinski, M.J. Sadowski, and J. Zebrowski and. Cherenkov emission provides detailed picture of non-thermal electron dynamics in the presence of magnetic islands. Nuclear Fusion, 55(12):123021, nov 2015.

[214] MunSeong Cheon, Junghee Kim, YoungHwa An, Dongcheol Seo, and Hyunseok Kim. Observation of the loss of pre-disruptive runaway electrons in KSTAR ohmic plasma disruptions. Nuclear Fusion, 56(12):126004, sep 2016.

[215] JD Strachan, EB Meservey, W Stodiek, RA Naumann, and F Girshick. Photo-neutron production in the plt tokamak. Nuclear Fusion, 17:140-144, 1977.

[216] Leonardo Pieroni and Sergio E Segre. Observation of non-maxwellian electron distribution functions in the alcator device by means of thomson scattering and their interpretation. Physical Review Letters, 34(15):928, 1975.

[217] GA Wurden, JA Oertel, and TE Evans. An in situ runaway electron diagnostic for diii-d. Review of Scientific Instruments, 85(11):11E111, 2014.

[218] AS Sharma and R Jayakumar. Runaway electrons during tokamak startup. Nuclear fusion, 28(3):491, 1988.

[219] DA Boyd, FJ Stauffer, and AW Trivelpiece. Synchrotron radiation from the atc tokamak plasma. Physical Review Letters, 37(2):98, 1976.

[220] H Knoepfel and SJ Zweben. High-energy runaway electrons in the oak ridge tokamak. Physical Review Letters, 35(20):1340, 1975.

[221] JD Strachan. Runaway electron transport in the lt-3 tokamak. Nuclear Fusion, 16(5):743, 1976.

[222] SJ Zweben, DW Swain, and HH Fleischmann. Radial transport of high-energy runaway electrons in ormak. Nuclear Fusion, 18(12):1679, 1978.

[223] O Ficker, J Mlynar, M Vlainic, J Cerovsky, J Urban, P Vondracek, V Weinzettl, E Macusova, J Decker, M Gospodarczyk, et al. Losses of runaway electrons in mhd-active plasmas of the compass tokamak. Nuclear Fusion, 57(7):076002, 2017. 
[224] R J Bickerton. Magnetic turbulence and the transport of energy and particles in tokamaks. Plasma Physics and Controlled Fusion, 39(3):339-365, mar 1997.

[225] R. S. Granetz, B. Esposito, J. H. Kim, R. Koslowski, M. Lehnen, J. R. Martin-Solis, C. PazSoldan, T. Rhee, J. C. Wesley, and L. Zeng. An itpa joint experiment to study runaway electron generation and suppressiona). Phys. Plasmas, 21(7):072506, Jul 2014.

[226] R.A. Tinguely, R.S. Granetz, M. Hoppe, and O. Embréus. Measurements of runaway electron synchrotron spectra at high magnetic fields in alcator c-mod. Nuclear Fusion, 58(7):076019, jun 2018.

[227] Z. Popovic, B. Esposito, J. R. Martín-Solís, W. Bin, P. Buratti, D. Carnevale, F. Causa, M. Gospodarczyk, D. Marocco, G. Ramogida, and M. Riva. On the measurement of the threshold electric field for runaway electron generation in the frascati tokamak upgrade. Physics of Plasmas, 23(12):122501, dec 2016.

[228] J. R. Martin-Solis, R. Sanchez, and B. Esposito. Experimental observation of increased threshold electric field for runaway generation due to synchrotron radiation losses in the ftu tokamak. Physical Review Letters, 105(18), Oct 2010.

[229] C. Paz-Soldan, N. W. Eidietis, R. Granetz, E. M. Hollmann, R. A. Moyer, J. C. Wesley, J. Zhang, M. E. Austin, N. A. Crocker, A. Wingen, and et al. Growth and decay of runaway electrons above the critical electric field under quiescent conditions. Phys. Plasmas, 21(2):022514, Feb 2014.

[230] EM Hollmann, N Commaux, NW Eidietis, TE Evans, DA Humphreys, AN James, TC Jernigan, PB Parks, EJ Strait, JC Wesley, et al. Experiments in diii-d toward achieving rapid shutdown with runaway electron suppression. Phys. Plasmas, 17(5):056117, 2010.

[231] P C de Vries, G Arnoux, A Huber, J Flanagan, M Lehnen, V Riccardo, C Reux, S Jachmich, C Lowry, G Calabro, and et al. The impact of the iter-like wall at jet on disruptions. Plasma Physics and Controlled Fusion, 54(12):124032, Nov 2012.

[232] VA Izzo, AN James, EM Hollmann, JH Yu, DA Humphreys, JC Wesley, LL Lao, PB Parks, PE Sieck, DG Whyte, et al. Runaway electron confinement modeling for diii-d, alcator c-mod and iter. In IAEA Fusion Energy Conference, 2010.

[233] R.D Gill. Generation and loss of runaway electrons following disruptions in jet. Nuclear Fusion, 33(11):1613-1625, Nov 1993.

[234] V Riccardo, G Arnoux, P Cahyna, TC Hender, A Huber, S Jachmich, V Kiptily, R Koslowski, 
L Krlin, M Lehnen, et al. Jet disruption studies in support of iter. Plasma Physics and Controlled Fusion, 52(12):124018, 2010.

[235] R Yoshino, S Tokuda, and Y Kawano. Generation and termination of runaway electrons at major disruptions in jt-60u. Nuclear Fusion, 39(2):151, 1999.

[236] Cedric Reux. Physics of the interaction between runaway electrons and the background plasma of the current quench in tokamak disruptions. In APS Meeting Abstracts, 2017.

[237] D. Shiraki, N. Commaux, L.R. Baylor, C.M. Cooper, N.W. Eidietis, E.M. Hollmann, C. PazSoldan, S.K. Combs, and S.J. Meitner. Dissipation of post-disruption runaway electron plateaus by shattered pellet injection in DIII-d. Nuclear Fusion, 58(5):056006, mar 2018.

[238] F Saint-Laurent, C Reux, J Bucalossi, A Loarte, S Bremond, C Gil, P Maget, Ph Moreau, and JL Segui. Disruption and runaways electron mitigation studies on tore supra. EXS, 2:16, 2010.

[239] C. Reux and et.al. N/a. submitted to Phys. Plasmas (2018), APS invited 2018., 2018.

[240] N. W. Eidietis, N. Commaux, E. M. Hollmann, D. A. Humphreys, T. C. Jernigan, R. A. Moyer, E. J. Strait, M. A. VanZeeland, J. C. Wesley, and J. H. Yu. Control of post-disruption runaway electron beams in diii-d. Phys. Plasmas, 19(5):056109, 2012.

[241] E.M. Hollmann, P.B. Parks, D.A. Humphreys, N.H. Brooks, N. Commaux, N. Eidietis, T.E. Evans, R. Isler, A.N. James, T.C. Jernigan, and et al. Effect of applied toroidal electric field on the growth/decay of plateau-phase runaway electron currents in diii-d. Nuclear Fusion, 51(10):103026, Sep 2011.

[242] B Esposito, L Boncagni, P Buratti, D Carnevale, F Causa, M Gospodarczyk, JR MartinSolis, Z Popovic, M Agostini, G Apruzzese, W Bin, C Cianfarani, R De Angelis, G Granucci, A Grosso, G Maddaluno, D Marocco, V Piergotti, A Pensa, S Podda, G Pucella, G Ramogida, G Rocchi, M Riva, A Sibio, C Sozzi, B Tilia, O Tudisco, and M Valisa and. Runaway electron generation and control. Plasma Physics and Controlled Fusion, 59(1):014044, nov 2016.

[243] G Pautasso, CJ Fuchs, O Gruber, CF Maggi, M Maraschek, T Pütterich, V Rohde, C Wittmann, E Wolfrum, P Cierpka, et al. Plasma shut-down with fast impurity puff on asdex upgrade. Nuclear Fusion, 47(8):900, 2007.

[244] M. Lehnen, A. Alonso, G. Arnoux, N. Baumgarten, S.A. Bozhenkov, S. Brezinsek, M. Brix, T. Eich, S.N. Gerasimov, A. Huber, and et al. Disruption mitigation by massive gas injection in jet. Nuclear Fusion, 51(12):123010, Nov 2011. 
[245] ZY Chen, WC Kim, YW Yu, AC England, JW Yoo, SH Hahn, SW Yoon, KD Lee, YK Oh, JG Kwak, et al. Study of runaway current generation following disruptions in kstar. Plasma Physics and Controlled Fusion, 55(3):035007, 2013.

[246] L. Zeng, H. R. Koslowski, Y. Liang, A. Lvovskiy, M. Lehnen, D. Nicolai, J. Pearson, M. Rack, P. Denner, K. H. Finken, and K. Wongrach and. Experimental observation of hot tail runaway electron generation in TEXTOR disruptions. Journal of Plasma Physics, 81(04), apr 2015.

[247] L Zeng, ZY Chen, YB Dong, HR Koslowski, Y Liang, YP Zhang, HD Zhuang, DW Huang, and X Gao. Runaway electron generation during disruptions in the j-text tokamak. Nuclear Fusion, 57(4):046001, 2017.

[248] C. Reux, J. Bucalossi, F. Saint-Laurent, C. Gil, P. Moreau, and P. Maget. Experimental study of disruption mitigation using massive injection of noble gases on tore supra. Nuclear Fusion, 50(9):095006, jul 2010.

[249] V.A. Izzo, E.M. Hollmann, A.N. James, J.H. Yu, D.A. Humphreys, L.L. Lao, P.B. Parks, P.E. Sieck, J.C. Wesley, R.S. Granetz, and et al. Runaway electron confinement modelling for rapid shutdown scenarios in diii-d, alcator c-mod and ITEr. Nuclear Fusion, 51(6):063032, May 2011.

[250] A. Matsuyama, M. Yagi, Y. Kagei, and N. Nakajima. Drift resonance effect on stochastic runaway electron orbit in the presence of low-order magnetic perturbations. Nuclear Fusion, 54(12):123007, nov 2014.

[251] S. S. Abdullaev, K. H. Finken, K. Wongrach, M. Tokar, H. R. Koslowski, O. Willi, and L. Zeng. Mechanism of runaway electron beam formation during plasma disruptions in tokamaks. Phys. Plasmas, 22(4):040704, Apr 2015.

[252] R Jaspers, NJ Lopes Cardozo, KH Finken, BC Schokker, G Mank, G Fuchs, and FC Schüller. Islands of runaway electrons in the textor tokamak and relation to transport in a stochastic field. Physical review letters, 72(26):4093, 1994.

[253] R. H. Tong, Z. Y. Chen, M. Zhang, D. W. Huang, W. Yan, and G. Zhuang. Observation of runaway electrons by infrared camera in j-TEXT. Review of Scientific Instruments, 87(11):11E113, aug 2016.

[254] N Commaux, Larry R Baylor, Stephen Kirk Combs, NW Eidietis, TE Evans, Charles R Foust, EM Hollmann, DA Humphreys, VA Izzo, AN James, et al. Novel rapid shutdown strategies for runaway electron suppression in diii-d. Nuclear Fusion, 51(10):103001, 2011. 
[255] M. Gobbin, L. Li, Y. Q. Liu, L. Marrelli, M. Nocente, G. Papp, G. Pautasso, P. Piovesan, M. Valisa, D. Carnevale, B. Esposito, L. Giacomelli, M. Gospodarczyk, P. J. McCarthy, P. Martin, W. Suttrop, M. Tardocchi, M. Teschke, and and. Runaway electron mitigation by 3d fields in the asdex-upgrade experiment. Plasma Phys. Control. Fusion, 60:014036, 2018.

[256] S. Putvinski, L. Baylor, D. Campbell, V. Chuyanov, Yu. Gribov, V. Leonov, A. Loarte, S. Maruyama, R. Pearce, R.A. Pitts, A. Polevoi, R. Mitteau, and M. Sugihara. Disruption mitigation in iter. Proceedings of the 23th IAEA Fusion Energy Conference, Daejon, Republic of Korea, ITER/1-6, 2010.

[257] G. Martin and F. Saint-Laurent. "fire 2nd report: Plasma experiments" cea internal report n.i00000504909. 2011, 2011.

[258] PB Parks and W Wu. Modelling penetration and plasma response of a dense neutral gas jet in a post-thermal quenched plasma. Nuclear Fusion, 54(2):023002, 2014.

[259] S.A. Bozhenkov, M. Lehnen, K.H. Finken, G. Bertschinger, H.R. Koslowski, D. Reiter, and R.C. Wolf and. Fuelling efficiency of massive gas injection in TEXTOR: mass scaling and importance of gas flow dynamics. Nuclear Fusion, 51(8):083033, jul 2011.

[260] J. Bucalossi, C. Reux, F. Saint-Laurent, M. Bécoulet, Y. Corre, P. Devynck, J.L. Gardarein, C. Gil, J. Gunn, G. Huysmans, P. Monier-Garbet, J.L. Ségui, and E. Tsitrone. Assessment of massive gas injection as a disruption mitigation tool in tore supra. Journal of Nuclear Materials, 415(1):S832-S835, aug 2011.

[261] AJ Thornton, KJ Gibson, JR Harrison, M Lehnen, R Martin, A Kirk, MAST Team, et al. Characterization of disruption mitigation via massive gas injection on MAST. Plasma Physics and Controlled Fusion, 54(12):125007, nov 2012.

[262] EM Hollmann, TC Jernigan, PB Parks, JA Boedo, TE Evans, M Groth, DA Humphreys, AN James, MJ Lanctot, D Nishijima, et al. Measurements of injected impurity assimilation during massive gas injection experiments in diii-d. Nuclear Fusion, 48(11):115007, 2008.

[263] Larry R Baylor, Stephen Kirk Combs, Charles R Foust, Thomas C Jernigan, SJ Meitner, PB Parks, John B Caughman, DT Fehling, S Maruyama, AL Qualls, et al. Pellet fuelling, elm pacing and disruption mitigation technology development for iter. Nuclear Fusion, 49(8):085013, 2009.

[264] N Commaux, Larry R Baylor, Thomas C Jernigan, EM Hollmann, PB Parks, DA Humphreys, JC Wesley, and JH Yu. Demonstration of rapid shutdown using large shattered deuterium 
pellet injection in diii-d. Nuclear Fusion, 50(11):112001, 2010.

[265] F Saint-Laurent, G Martin, T Alarcon, A Le Luyer, PB Parks, P Pastor, S Putvinski, C Reux, J Bucalossi, S Bremond, et al. Overview of runaway electron control and mitigation experiments on tore supra and lessons learned in view of iter. Fusion Science and Technology, 64(4):711-718, 2013.

[266] B Bazylev, G Arnoux, W Fundamenski, Yu Igitkhanov, M Lehnen, and JET EFDA Contributors. Modeling of runaway electron beams for jet and iter. Journal of Nuclear Materials, 415(1):S841-S844, 2011.

[267] EM Hollmann, G Arnoux, N Commaux, NW Eidietis, TE Evans, RS Granetz, A Huber, DA Humphreys, VA Izzo, AN James, et al. Plasma-surface interactions during tokamak disruptions and rapid shutdowns. Journal of Nuclear Materials, 415(1):S27-S34, 2011.

[268] C. Reux, V. Plyusnin, B. Alper, D. Alves, B. Bazylev, E. Belonohy, A. Boboc, S. Brezinsek, I. Coffey, J. Decker, and et al. Runaway electron beam generation and mitigation during disruptions at jet-ilw. Nuclear Fusion, 55(9):093013, Aug 2015.

[269] J. R. Martin-Solis, A. Loarte, E.M. Hollmann, B. Esposito, and V. Riccardo. Inter-machine comparison of the termination phase and energy conversion in tokamak disruptions with runaway current plateau formation and implications for iter. Nuclear Fusion, 54(8):083027, Jul 2014.

[270] L.R. Baylor, C. C. Barbier, J. R. Carmichael, S. K. Combs, M. N. Ericson, N. D. Bull Ezell, P. W. Fisher, M. S. Lyttle, S. J. Meitner, D. A. Rasmussen, S. F. Smith, J. B. Wilgen, S. Maruyama, and G. Kiss. Disruption mitigation system developments and design for ITER. Fusion Science and Technology, 68(2):211-215, sep 2015.

[271] V Leonov, V Zhogolev, S Konovalov, and M Lehnen. Simulation of the pre-thermal quench stage of disruptions during massive gas injection and projections for iter. In Proceedings of the 25th IAEA Fusion Energy Conference, St. Petersburg, Russian Federation, 2014.

[272] D. Hu, E. Nardon, M. Lehnen, G.T.A. Huijsmans, and D.C. van Vugt and. 3d non-linear MHD simulation of the MHD response and density increase as a result of shattered pellet injection. Nuclear Fusion, 58(12):126025, oct 2018.

[273] Charlson Kim, Paul Parks, Lang Lao, Michael Lehnan, Alberto Loarte, Valerie Izzo, and Nimrod Team. Shattered pellet injection simulations with nimrod. In APS Meeting Abstracts, 2017. 
[274] M Sugihara, M Shimada, H Fujieda, Yu Gribov, K Ioki, Y Kawano, R Khayrutdinov, V Lukash, and J Ohmori. Disruption scenarios, their mitigation and operation window in ITER. Nuclear Fusion, 47(4):337-352, mar 2007.

[275] JC Wesley, AW Hyatt, EJ Strait, DP Schissel, SM Flanagan, RS Granetz, M Johnson, Y Kawano, J Lister, R Martin, et al. Disruption characterization and database activities for iter. In Proc. 21st IAEA Fusion Energy Conf., Chengdu, China, 2006.

[276] M Sugihara, S Putvinski, DJ Campbell, S Carpentier-Chouchana, F Escourbiac, S Gerasimov, Yu Gribov, TC Hender, T Hirai, K Ioki, et al. Disruption impacts and their mitigation target values for iter operation and machine protection. In Proceedings of the 24th IAEA Fusion Energy Conference, San Diego, USA, pages 8-13, 2012.

[277] D. Shiraki, N. Commaux, L.R. Baylor, N.W. Eidietis, E.M. Hollmann, V.A. Izzo, R.A. Moyer, and C. Paz-Soldan. Characterization of MHD activity and its influence on radiation asymmetries during massive gas injection in DIII-d. Nuclear Fusion, 55(7):073029, jun 2015.

[278] S Jachmich, P Drewelow, S Gerasimov, U Kruezi, M Lehnen, C Reux, V Riccardo, I Carvalho, A Pau, M Imsirek, et al. Disruption mitigation at jet using massive gas injection. In Proc. 43rd European Physical Society Conf. on Plasma Physics (EPS)(Leuven, Belgium, 2016), volume 40, 2016.

[279] M Lehnen et al. Plasma disruption management in iter. In Proceedings of the 26th IAEA Fusion Energy Conference, Kyoto, Japan, EX/P6-39, 2016.

[280] G F Matthews, B Bazylev, A Baron-Wiechec, J Coenen, K Heinola, V Kiptily, H Maier, C Reux, V Riccardo, F Rimini, G Sergienko, V Thompson, and A Widdowson and. Melt damage to the JET ITER-like wall and divertor. Physica Scripta, T167:014070, jan 2016.

[281] A. Loarte, P. Andrew, G.F. Matthews, J. Paley, V. Riccardo, T. Eich, C. Fuchs, O. Gruber, A. Herrmann, G. Pautasso, G. Counsell, G. Federici, K.H. Finken, G. Maddaluno, D. Whyte, et al. Expected energy fluxes onto iter plasma facing components during disruption thermal quenches from multi-machine data comparisons. Proceedings of the 20th IAEA Fusion Energy Conference, Villamoura, Portugal, IT/P3-34, 2004.

[282] M. Lehnen. Challenges of disruption mitigation in iter. In 44th EPS Conference on Plasma Physics, Belfast, UK, I4.008, 2017.

[283] M Lehnen et al. R\&d for reliable disruption mitigation in iter. In Proceedings of the 27th IAEA Fusion Energy Conference, Ahmedabad, India, 2018. 
[284] Santosh P Pandya, Laura Core, Robin Barnsley, Joël Rosato, Roger Reichle, Michael Lehnen, Luciano Bertalot, and Michael Walsh. Modeling of bremsstrahlung emission from the confined runaway electrons and applications to the hard x-ray monitor of ITER. Physica Scripta, 93(11):115601, oct 2018 .

[285] G. Taylor, M. E. Austin, A. Basile, J. H. Beno, S. Danani, R. Feder, S. Houshmandyar, A. E. Hubbard, D. W. Johnson, A. Khodak, R. Kumar, S. Kumar, A. Ouroua, S. B. Padasalagi, H. K. B. Pandya, P. E. Phillips, W. L. Rowan, J. Stillerman, S. Thomas, V. S. Udintsev, G. Vayakis, M. Walsh, and D. Weeks. Update on the status of the ITER ECE diagnostic design. EPJ Web of Conferences, 147:02003, 2017.

[286] R. Reichle, B. Beaumont, D. Boilson, R. Bouhamou, M.-F. Direz, A. Encheva, M. Henderson, R. Huxford, F. Kazarian, Ph. Lamalle, S. Lisgo, R. Mitteau, K. M. Patel, C. S. Pitcher, R. A. Pitts, A. Prakash, R. Raffray, B. Schunke, J. Snipes, A. Suarez Diaz, V. S. Udintsev, C. Walker, and M. Walsh. Concept development for the ITER equatorial port visible/infrared wide angle viewing system. Review of Scientific Instruments, 83(10):10E520, oct 2012.

[287] Yunong Wei, Zhongyong Chen, Duwei Huang, Ruihai Tong, and Xiaolong Zhang. Formation and dissipation of runaway current by mgi on j-text. In APS Meeting Abstracts, 2017.

[288] G Papp, G Pautasso, J Decker, M Gobbin, PJ McCarthy, D Choi, S Coda, B Duval, R Dux, B Erdos, et al. Runaway electron generation and mitigation on the european medium sized tokamaks asdex upgrade and tcv. In Proceedings of the 26th IAEA Fusion Energy Conference, Kyoto, Japan, TH/7-1, 2016.

[289] C Paz-Soldan, CM Cooper, P Aleynikov, NW Eidietis, A Lvovskiy, DC Pace, DP Brennan, EM Hollmann, C Liu, RA Moyer, et al. Resolving runaway electron distributions in space, time, and energy. Phys. Plasmas, 25(5):056105, 2018.

[290] L. Zeng, H. R. Koslowski, Y. Liang, A. Lvovskiy, M. Lehnen, D. Nicolai, J. Pearson, M. Rack, H. Jaegers, K. H. Finken, and et al. Experimental observation of a magnetic-turbulence threshold for runaway-electron generation in the textor tokamak. Physical Review Letters, 110(23), Jun 2013.

[291] A J Dai, Z Y Chen, D W Huang, R H Tong, J Zhang, Y N Wei, T K Ma, X L Wang, H Y Yang, H L Gao, and Y Pan and. Conversion of magnetic energy to runaway kinetic energy during the termination of runaway current on the j-TEXT tokamak. Plasma Physics and Controlled Fusion, 60(5):055003, mar 2018. 
[292] Victor Lukash, A.A. Kavin, Y Gribov, R.R. Khayrutdinov, and Alberto Loarte. Study of iter plasma position control during disruptions with formation of runaway electrons. 40th EPS Conference on Plasma Physics, EPS 2013, 2:1486-1489, 012013. 